OPEN ACCESS

Edited by:

António Pestana Morais,

University of Aveiro, Portugal

Reviewed by:

Zhenbin Wu,

University of lllinois at Chicago,

United States

Joel Wesley Walker,

Sam Houston State University,

United States

*Correspondence:

Tomás E. Gonzalo

tomas.gonzalo@monash.edu

Specialty section:

This article was submitted to

High-Energy and Astroparticle

Physics,

a section of the journal

Frontiers in Physics

Received: 15 December 2018

Accepted: 02 May 2019

Published: 04 June 2019

Citation:

Croon D, Gonzalo TE, Graf L, Košnik N and White $G$ (2019) GUT Physics in the Era of the LHC. Front. Phys. 7:76. doi: 10.3389/fphy.2019.00076

\section{GUT Physics in the Era of the LHC}

\author{
Djuna Croon ${ }^{1}$, Tomás E. Gonzalo ${ }^{2,3 *}$, Lukas Graf ${ }^{4,5}$, Nejc Košnik ${ }^{6,7}$ and Graham White ${ }^{1}$ \\ ${ }^{1}$ TRIUMF Theory Group, Vancouver, BC, Canada, ${ }^{2}$ Department of Physics, University of Oslo, Oslo, Norway, ${ }^{3}$ ARC Centre of \\ Excellence for Particle Physics at the Tera-Scale, School of Physics and Astronomy, Monash University, Melbourne, VIC, \\ Australia, ${ }^{4}$ Department of Physics and Astronomy, University College London, London, United Kingdom, \\ ${ }^{5}$ Max-Planck-Institut für Kernphysik, Heidelberg, Germany, ${ }^{6}$ Department of Physics, University of Ljubljana, Ljubljana, \\ Slovenia, ${ }^{7}$ Jožef Stefan Institute, Ljubljana, Slovenia
}

Grand Unified Theories (GUTs) are one of the most interesting high-energy completions of the Standard Model, because they provide a rich, powerful and elegant group-theoretical framework able to resolve a variety of problems remaining in our current understanding of particle physics. They usually act as motivators for many low energy BSM theories, such as left-right symmetric or supersymmetric models, and they serve to fill the gap between the experimentally reachable low energies and the physics in the ultraviolet. In recent years, however, they have fallen slightly from the spotlight, in favor of "simplified" models with more specific phenomenological predictions. The aim of this review is to summarize the state of the art on GUTs and argue for their importance in modern physics. Recent advances in experiments permit to test the predictions of GUTs at different energy scales. First, as GUTs can play a role in the inflationary dynamics of the early Universe, their imprints could be found in the CMB observations by the Planck satellite. Remarkably enough, GUTs could manifest themselves also in terrestrial tests; several planned experiments aim to probe the proton stability and to establish order of magnitude higher bounds on its lifetime. Moreover, the predictions of specific GUT models could be tested even at the LHC thanks to its high energy reach, via searches for exotic states or additional contributions to flavor anomalies.

Keywords: grand unified theories, supersymmetry, colliders, neutrinos, cosmology, gravitational waves, flavour, leptoquarks

\section{INTRODUCTION}

The Standard Model (SM) [1-3] of particle physics is an incredible successful theory of subatomic physics. It describes the electroweak and strong interactions of fundamental particles with surprising accuracy up to the energy scales of modern day experiments. Further supported by the discovery of the Higgs boson [4,5], it stands as one of the best evidences that symmetries and the mechanism of spontaneous symmetry breaking play a critical role on the Universe at the smallest scales [6-8].

In spite of its success at explaining with astonishing precision most of the observed phenomena, the SM cannot be the ultimate theory of particle physics. The Higgs quartic coupling in the SM becomes negative at scales $\gtrsim 10^{10} \mathrm{GeV}$, rendering the vacuum state of the theory unstable at high energies [9]. New physics must then surface below or around that scale to stabilize the vacuum. Furthermore, there is a continuously increasing amount of observations that are in tension with the predictions of the SM. From the discovery of neutrino oscillations $[10,11]$ to the recent anomalies in the flavor sector [12-14], these phenomena cannot be explained with the SM alone and contributions from beyond the SM (BSM) physics may be required to accommodate them. 
Grand Unified Theories (GUTs) [15-19] are well motivated extensions of the SM that can address several of its outstanding issues. As the SM does for electromagnetism and weak nuclear decays, GUTs exploit the power of symmetries and group theory to unify the electroweak and strong interactions into a single force. As can be noticed in Figure 1, the flow of the SM gauge couplings already hints at a possible unification at a high scale, thereby providing further motivation for GUTs as appealing BSM models.

The predicted unification of forces provides an explanation for the ad hoc nature of the SM charge assignments and their accidental anomaly cancellation [20, 21]. Through the introduction of new fields and symmetries, GUTs can resolve many of the issues of the SM: they can provide an explanation for the lightness of neutrino masses, as well as introducing additional contributions that can accommodate some of the observed flavor anomalies. In addition, GUTs can also live alongside other BSM models, such as Supersymmetry (SUSY), with both theories complementing each other and on the whole becoming a better candidate for a successful BSM theory [22].

Naturally living at high energies, it is expected that GUTs have some observable consequences for the cosmological evolution of the Universe, as they can play a role during the inflationary epoch and their phase transitions may be the source for matterantimatter asymmetry or gravitational waves [23]. With or without SUSY, GUTs also make predictions that can be tested at low energy experiments such as particle colliders, which can search for new exotic states or deviations on flavor observables. Some of its high energy repercussions can also be probed with precision experiments, with nucleon decay measurements at the forefront. In short, Grand Unified Theories have observable effects in many fronts and can be probed by current and upcoming experiments in the near future.

Therefore, throughout this review we will focus on the description of GUT models and their observable consequences. We will introduce the basic concepts and summarize some of the modern research in GUTs. Out of all the possible observable

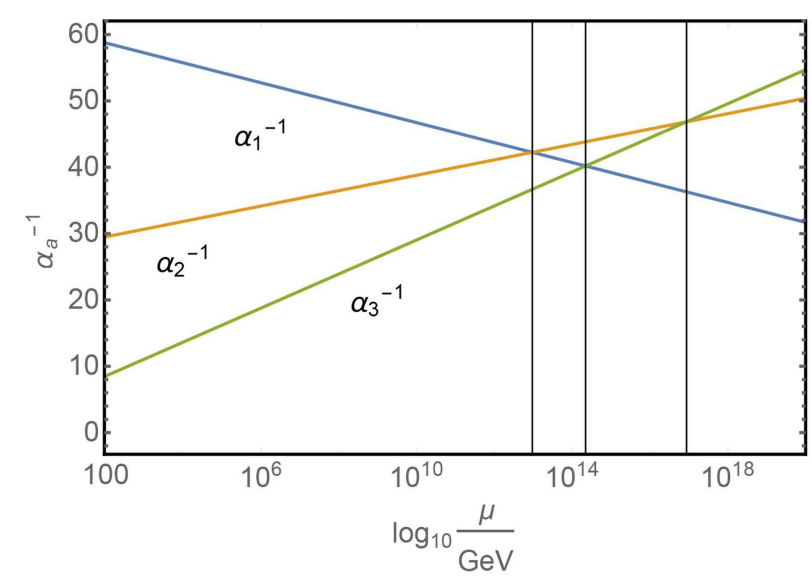

FIGURE 1 | Renormalization group flow of the standard model gauge couplings. probes of GUTs, we will focus on a subset of them. In the cosmological front we will outline the advances on inflationary GUTs, detail their observable gravitational wave signatures, from cosmic strings and phase transitions, and their relation with mechanisms for baryo and leptogenesis. The low energy front will cover collider searches for supersymmetry, leptoquarks and exotic states. Lastly, a number of precision tests of unification will be detailed, including nucleon decay, flavor observables and neutrinoless double beta decay.

As ultraviolet (UV) completions of the Standard Model that live at very high energies, GUTs are often closely related to theories of gravity, such as string theory. In fact, many unified theories arise as four-dimensional compactifications in some realization of superstring theory $[24,25]$. However, throughout this review we will not concern ourselves with these string theory realizations. For a review on embedding GUTs in the heterotic string and outcomes of string compactification for unified theories see Raby [22].

\section{BASICS ON GUT MODELS}

Grand Unified Theories are extensions of the SM with larger symmetry groups. Strictly speaking, GUTs require that the unified group be a simple group, e.g., $S U(5), S O(10)$, or $E_{6}$. However, here we use the term GUT more loosely, referring to any extension of the SM symmetries including product groups such as $S U(5) \times U(1)$ and $S U(4) \times S U(2) \times S U(2)$. Candidate groups for a realistic GUT model must satisfy two conditions: they must contain the SM group as a subgroup and they must have complex representations that reproduce the chiral structure of the SM. Although groups with pseudoreal representations have been studied as candidates for unified theories, $E_{7}$ [26], we will not consider them here.

\section{1. $S U(5)$}

The first appearance of a GUT in the literature dates back to 1974 when H. Georgi and S. Glashow proposed the unification of the SM gauge group into a simple group, $S U(5)$ [15]. In their proposal all the left-handed fermions of a single generation fell into two representations of the group, $\overline{\mathbf{5}}$ and $\mathbf{1 0}$, in the following way

$$
\overline{\mathbf{5}} \leftrightarrow\left(\begin{array}{c}
d_{1}^{c} \\
d_{2}^{c} \\
d_{3}^{c} \\
e \\
-v
\end{array}\right), \quad \mathbf{1 0} \leftrightarrow\left(\begin{array}{ccccc}
0 & u_{3}^{c} & -u_{2}^{c} & u_{1} & d_{1} \\
-u_{3}^{c} & 0 & u_{1}^{c} & u_{2} & d_{2} \\
u_{2}^{c} & -u_{1}^{c} & 0 & u_{3} & d_{3} \\
-u_{1} & -u_{2} & -u_{3} & 0 & e^{c} \\
-d_{1} & -d_{2} & -d_{3} & -e^{c} & 0
\end{array}\right) .
$$

and the gauge and Higgs sector of the theory were embedded into the $\mathbf{2 4}$ and 5 representations, respectively. In addition to the SM Higgs boson present in the representation $\mathbf{5}$, often a scalar $\overline{\mathbf{5}}$ representation is also present, which contains a second $S U(2)_{L}$ doublet, necessary for UV completions of two-Higgs doublet models (2HDM) [27].

The Georgi-Glashow (GG) model was the first attempt of a fully-unified model for particle physics, and it provided a neat explanation for the hypercharge quantization in the SM. The 
traceless hypercharge generator can be written as [21]

$$
Y=\alpha \operatorname{diag}\left(-\frac{1}{3},-\frac{1}{3},-\frac{1}{3}, \frac{1}{2}, \frac{1}{2}\right)
$$

which when acting upon the representations of $S U(5)$ results in the specific hypercharge assignments of the SM fields, i.e., for $\alpha=1, Y(Q)=1 / 6, Y(L)=-1 / 2, Y\left(u^{c}\right)=-2 / 3, Y\left(d^{c}\right)=1 / 3$ and $Y\left(e^{c}\right)=1$. In unified theories one often uses the "GUT normalization" of the hypercharge, which corresponds simply to a rescaling of the charges and gauge couplings as $Y_{G U T}=\sqrt{3 / 5} \mathrm{Y}$ and $g_{1}=\sqrt{5 / 3} g^{\prime}$ [28].

Spontaneous symmetry breaking of $S U(5)$ happens when a scalar field in a non-trivial representation of the group acquires a vacuum expectation value (vev). The minimal representation of $S U(5)$ that can achieve this goal while keeping the SM phase unbroken is the $\mathbf{2 4}[15,29]$. This vev provides a mass to the off-diagonal SU(5) gauge bosons while the SM gauge bosons remain massless.

By virtue of the unification into a single gauge group, the GG model requires strict unification of the SM gauge couplings, which is hinted at but not really achieved in the SM [28, 29], as can be seen in Figure 1, as well as that of Yukawa couplings for each of the two representations, a difficult task in its minimal version $[30,31]$.

The minimal $S U(5)$ version suffers from other afflictions beyond the failed gauge and Yukawa unification mentioned above. One of these is the introduction of an artificial hierarchy, known as doublet-triplet splitting [32,33], in the components to the Higgs representation 5. The colored components must be quite heavy to avoid rapid proton decay whereas the uncolored components must be relatively light, for they correspond to the SM Higgs doublet, now know to have a mass of $m_{h}=125.18$ $\mathrm{GeV}$ [34]. Solutions to this problem in several SU(5) models have been proposed, such as the "missing partner mechanism" [33, 35] or the "double missing partner mechanism" [36, 37].

Another case where the minimal $S U(5)$ falls short is the lack of a mechanism for the generation of neutrino masses. Extended scalar sectors can generate neutrino masses in the type-I [38] and type-III [39] seesaw mechanisms ${ }^{1}$, but the resulting theories are often non-renormalisable. Renormalisable $S U(5)$ models can also be constructed where the neutrino masses are generated via a mixture of type-I and type-III seesaw [40] or the Zee mechanism $[41,42]$.

Worst of all, however, is the fact that the vanilla $S U(5)$ model predicts rapid proton decay. The lifetime of the proton can be naively estimated as [30]

$$
\tau_{p} \sim \frac{M_{X}^{4}}{m_{p}^{5}},
$$

with $m_{p}$ the mass of the proton and $M_{X}$ the mass of the mediator field at the scale of unification. The apparent unification of gauge couplings happens at an energy scale $\mu \sim 10^{15} \mathrm{GeV}$, which gives a half life for the proton of the order of $10^{31}$ years, far below

\footnotetext{
${ }^{1}$ See section 3.2 for details on neutrino mass generation through the seesaw mechanism.
}

the experimental bound from the Super-Kamiokande experiment of $1.6 \times 10^{34}$ years [43]. Particular choices of the Higgs sector of the $S U(5)$ model, however, avoid this issue, rendering nonminimal $S U(5)$ models viable candidates [44-46]. Furthermore, $S U(5)$ models with vector-like fermions can be consistent with current limits and even predict an upper bound on the lifetime of proton decay [47].

One of the fundamental issues with GUT models, which remains as a concern today, is the gauge hierarchy problem. The large hierarchy between the mass scale of unification and the electroweak scale poses a problem since it causes large loop corrections to the Higgs mass [48]. Supersymmetry (SUSY) was proposed as a solution to this issue [49] and even acted as a motivation for unified theories since some of its minimal realizations, such as the MSSM, predicted the unification of gauge couplings, as can be seen in Figure 2.

Supersymmetric GUTs are in fact rather popular and have in some cases been proven to be more successful at representing nature than regular GUTs [50]. In SUSY SU(5) theories the scale of unification is typically larger than in non-supersymmetric models ${ }^{2}$, around $\mu \sim 2 \times 10^{16} \mathrm{GeV}$ as can be seen in Figure 2. This has two advantageous consequences for these models: the larger mass scale for the gauge mediators imposes a further suppression on nucleon decay processes, consistent with experimental measurements [50]; and pushes the unification scale beyond the scale of inflation, which helps to dilute the magnetic monopoles naturally present in the theory [52]. Another issue in vanilla $S U(5)$ models that can be addressed in its supersymmetric version is the doublet-triplet splitting, where the Higgs doublets are made light via cancellations of the superpotential parameters $[32,33,53,54]$.

\subsection{Flipped $S U(5)$}

An alternative solution to the issues of $S U(5)$ models, without supersymmetry, is what is now known as flipped $S U(5)[55,56]$. The flipped version differs from regular $S U(5)$ in its gauge group, extended to $S U(5) \times U(1)$, and the manner in which the SM particle fields are embedded into representations of the group. In contrast to Equation (1), the matter representations in the flipped $S U(5)$ model are

$\overline{\mathbf{5}} \leftrightarrow\left(\begin{array}{c}u_{1}^{c} \\ u_{2}^{c} \\ u_{3}^{c} \\ e \\ -v\end{array}\right), \quad \mathbf{1 0} \leftrightarrow\left(\begin{array}{ccccc}0 & d_{3}^{c} & -d_{2}^{c} & u_{1} & d_{1} \\ -d_{3}^{c} & 0 & d_{1}^{c} & u_{2} & d_{2} \\ d_{2}^{c} & -d_{1}^{c} & 0 & u_{3} & d_{3} \\ -u_{1} & -u_{2} & -u_{3} & 0 & v^{c} \\ -d_{1} & -d_{2} & -d_{3} & -v^{c} & 0\end{array}\right), \quad \mathbf{1} \leftrightarrow\left(e^{c}\right)$

where $v^{c}$ labels the right-handed neutrino field, whose presence provides a mechanism for neutrino mass generation, which was absent in vanilla $S U(5)$.

With these different embeddings of the SM fields, the hypercharge operator is no longer contained in $S U(5)$, as in Equation (2), but it is rather a combination of diagonal generators of both $S U(5)$ and $U(1)$. With standard normalization the

${ }^{2}$ A detailed description of unification in SUSY SU(5) can be found in Dorsner et al. [51]. 


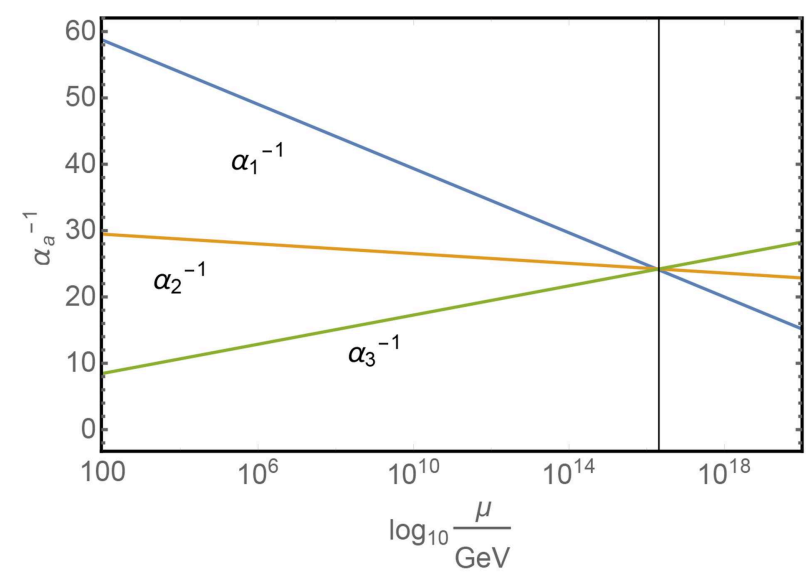

FIGURE 2 | Renormalization Group flow of the MSSM gauge couplings.

hypercharge operator can be written as [57]

$$
Y=-\frac{1}{5} T_{24}+\frac{1}{5} X
$$

where $T_{24}$ is a diagonal generator of $S U(5)$ and $X$ the $U(1)$ charge.

Due to the extended gauge sector and modified unified conditions of flipped $S U(5)$, proton decay does not become an issue [57]. In addition, in flipped $S U(5)$ magnetic monopoles cannot be created since the supergroup containing the charge operator is not a simple group [52, 57].

As was the case with regular $S U(5)$ models, flipped $S U(5)$ can be extended with the help of supersymmetry. The combination of solutions to the issues of the SU(5) model that both SUSY and flipped $S U(5)$ offer makes SUSY flipped $S U(5)$ one of the most popular GUTs in the literature $[58,59]$, in spite of not realizing a full unification of gauge couplings.

Flipped $S U(5)$ models are also well motivated from their UV completions, since they can easily be derived naturally from weakly-coupled string theory. As opposed to vanilla $S U(5)$, which undergoes symmetry breaking via a 24-dimensional representation, the flipped $S U(5)$ model does not require large dimensional representations, as it breaks via a $\mathbf{1 0}_{1}$, and it is therefore easier to obtain from a manifold compactification of string theory $[60,61]$.

\subsection{Pati-Salam and the Left-Right Symmetry}

Around the same time that the $S U(5)$ model was proposed, R. Pati and A. Salam suggested another extension of the SM [16]. They proposed a rearrangement of the SM fields into a different group configuration, $S U(4)_{c} \times S U(2)_{L} \times S U(2)_{R}$. Though not really a fully unified theory, it provided a partial unification of leptons and quarks into a single color group, $S U(4)_{c}$, while at the same time introducing another copy of $S U(2)$ for the righthanded sector of the theory. This model automatically contains a right-handed neutrino, embedded into a $S U(2)_{R}$ doublet with the right-handed charged lepton. Thus, the SM fields fall into two representations of the group in the following way

$$
\begin{array}{r}
\{\mathbf{4}, \mathbf{2}, \mathbf{1}\} \leftrightarrow\left(\begin{array}{cccc}
u_{1} & u_{2} & u_{3} & v \\
d_{1} & d_{2} & d_{3} & e
\end{array}\right), \\
\left\{\overline{\mathbf{4}}, \mathbf{1}, 2^{*}\right\} \leftrightarrow\left(\begin{array}{cccc}
d_{1}^{c} & d_{2}^{c} & d_{3}^{c} & e^{c} \\
-u_{1}^{c} & -u_{2}^{c} & -u_{3}^{c} & -v^{c}
\end{array}\right) .
\end{array}
$$

One of the major successes of the Pati-Salam (PS) model was being the first appearance of a left-right symmetric model, with a right-handed sector $S U(2)_{R}$ and a heavy right-handed gauge boson $W_{R}$ [62]. It was also the original proposal for the idea of quark-lepton complementarity. As an amalgamation of the two ideas, the PS group maximally contains the left-right symmetry group, $S U(3)_{c} \times S U(2)_{L} \times S U(2)_{R} \times U(1)_{B-L}[17,63]$, as well as the quark-lepton unified group, $S U(4)_{c} \times S U(2)_{L} \times U(1)_{R}[64,65]$.

Left-right symmetric models, à la Pati-Salam or of the type $S U(3)_{c} \times S U(2)_{L} \times S U(2)_{R} \times U(1)_{B-L}$, are fairly popular because they naturally include a right-handed neutrino and can generate light neutrino masses via some type of seesaw mechanism [66, 67]. Similar to PS, left-right symmetric (LR) models are not fully unified theories, yet they can be an intermediate step on the breaking chain of a PS model [68] or some other unified theory [69].

Symmetry breaking in the PS model can happen through a number of different paths, depending on the vev of the scalar fields in the theory. The most compelling paths preserve either the LR symmetry, with the LR group $S U(3)_{c} \times S U(2)_{L} \times S U(2)_{R} \times$ $U(1)_{B-L}$ as an intermediate step, or quark-lepton unification, with $S U(4)_{c} \times S U(2)_{L} \times U(1)_{R}$ as a subgroup [70]. Further symmetry breaking from the LR symmetry model happens when either a pair of $S U(2)$ doublets (one left-handed and one righthanded) $[62,71]$, or a pair of $S U(2)$ triplets (left and righthanded) [68] acquire a vev. In both PS and LR theories, the hypercharge operator is written as a linear combination of the diagonal $S U(2)_{R}$ generator and the $B-L$ charge $\left[U(1)_{B-L}\right.$ generator embedded in $S U(4)_{c}$ in PS] as

$$
Y=T_{R}^{3}+\frac{1}{2}(B-L)
$$

As opposed to the case of $S U(5)$ the proton is often stable in PS and LR models. This occurs because the gauge sector of the theory preserves $B$ and $L$ number independently and the only scalar fields that can mediate the transition are in antisymmetric representations, rarely seen in PS or LR models [68].

The addition of supersymmetry to PS and left-right symmetric models [72] is not as straightforward as with other GUT models. The simplest scenario with both SUSY and LR symmetry was shown to fail to achieve spontaneous symmetry breaking [73]. In order to circumvent this issue one must either add extra fields, such as a parity-odd singlet $^{3}$ [74] or an extra Higgs field [75], or alternatively supplement the Lagrangian with nonrenormalizable operators [76]. One of the main advantages of

\footnotetext{
${ }^{3}$ Although successful in achieving spontaneous symmetry breaking (SSB) in this SUSY LR model, the resulting vacuum state does not preserve the electromagnetic charge.
} 
SUSY LR models, and the reason why so much effort is put on resolving the SSB issue, is that they naturally preserve $R$-parity, since $B-L$ is a gauge symmetry of the theory, which forbids the dangerous baryon and lepton number violating operators that appear in the MSSM, thereby making the lightest SUSY particle stable [77].

\section{4. $S O(10)$}

Although the GG and PS models seem quite distinct in their approach to unification, they have a common ancestor. Both $S U(5) \times U(1)$ and $S U(4) \times S U(2) \times S U(2)$ are maximal subgroups of another Lie group of larger dimension, $S O(10)$. This was first realized by Fritzsch and Minkowski [18], and independently by Georgi [19], who proposed a model of unification with several intermediate steps. $S O(10)$ has since been the most popular choice as a unification group, since it provides a vast display of options for field configurations and symmetry breaking patterns.

One of the many key features of $S O(10)$ models is that they fully unify a generation of SM fermions into a single representation of the group. Thus, the 16 Weyl fermions, including right-handed neutrinos, can be embedded into the fundamental 16 representation of $S O(10)$ as

$$
\mathbf{1 6}=\left\{u_{1}^{c}, d_{1}^{c}, d_{1}, u_{1}, v^{c}, e^{c}, d_{2}, u_{2}, u_{2}^{c}, d_{2}^{c}, d_{3}, u_{3}, u_{3}^{c}, d_{3}^{c}, e, v\right\}_{L} .
$$

Due to the transformation properties of the $S O(10)$ group, the spinor representation $\mathbf{1 6}$ is a complex representation, thereby satisfying chirality as observed in the Standard Model. Additionally $S O(10)$ is a "safe algebra" [78], it does not suffer from anomalies as, for example, the $S U(5)$ case above, which makes model building in $S O(10)$ easier for it does not rely on some specific field configurations to cancel the gauge anomalies [79].

Despite the large amount of $S O(10)$ models in the literature, a common feature is that the gauge fields are embedded in the adjoint representation of the group, that is $\mathbf{4 5}$,

$$
\begin{aligned}
\mathbf{4 5} & \rightarrow\{\mathbf{8}, \mathbf{1}, 0\} \oplus\{\mathbf{1}, \mathbf{3}, 0\} \oplus\{\mathbf{1}, \mathbf{1}, 0\} \quad \leftarrow \text { SM gauge bosons } \\
& \oplus\left\{\mathbf{3}, \mathbf{2}, \frac{1}{6}\right\} \oplus\left\{\overline{\mathbf{3}}, \mathbf{2},-\frac{1}{6}\right\} \oplus\left\{\mathbf{3}, \mathbf{2}, \frac{1}{6}\right\} \\
& \left.\oplus\left\{\overline{\mathbf{3}}, \mathbf{2},-\frac{1}{6}\right\} \oplus\left\{\overline{\mathbf{3}}, \mathbf{1},-\frac{2}{3}\right\} \oplus\left\{\mathbf{3}, \mathbf{1}, \frac{2}{3}\right\}\right\} \leftarrow \text { leptoquarks } \\
& \oplus\{\mathbf{1}, \mathbf{1}, 1\} \oplus\{\mathbf{1}, \mathbf{1},-1\} \oplus\{\mathbf{1}, \mathbf{1}, 0\} .
\end{aligned}
$$

which contains the SM gauge bosons as well as off-diagonal components which, as happened in $S U(5)$, can mediate quarklepton transitions, known as leptoquarks. The Yukawa sector in $S O(10)$ models is often also quite recurrent because, at the renormalizable level, it can only be of the form [80]

$$
\begin{array}{r}
\mathcal{L}_{Y u k}=\mathrm{Y} \cdot \mathbf{1 6}^{T} C_{L} C_{10}\left(\Gamma_{i} \Phi^{i}+\Gamma_{[i} \Gamma_{j} \Gamma_{k]} \Phi^{i j k}+\right. \\
\left.\Gamma_{[i} \Gamma_{j} \Gamma_{k} \Gamma_{l} \Gamma_{m]} \Phi^{i j k l m}\right) \mathbf{1 6}
\end{array}
$$

where $\mathrm{Y}$ is the matrix of Yukawa couplings, $C_{L}$ and $C_{10}$ the charge conjugation matrices in the Poincaré and $S O(10)$ groups, $\Gamma_{i}$ the generators of $S O(10)$ in the spinor representation and $\Phi^{i}, \Phi^{i j k}$, and $\Phi^{i j k l m}$ are scalar fields in the 10, 120, and $\overline{\mathbf{1 2 6}}$ representations, respectively. The SM Higgs field is, therefore, some linear combination of these fields and hence the SM fermion masses directly follow from the Yukawa matrix $\mathbf{Y}$ and the vacuum expectation values of the scalar fields. The particular choice of the scalar sector is typically guided by the principle of minimality. While the minimal regular (non-SUSY) $S O(10)$ model with SSB driven by the $\mathbf{4 5}$ and $\mathbf{1 2 6}$ Higgs representations has been revived and still represents a phenomenologically viable scenario [81-85], this is not the case of the minimal SUSY SO(10) model $[86,87]$.

Symmetry breaking in $S O(10)$ models can occur through one of many different paths. Since both $S U(5) \times U(1)$ and $S U(4) \times S U(2) \times S U(2)$ are subgroups of $S O(10)$, they can be an intermediate step on the symmetry breaking path toward the Standard Model, as can be any of their respective subgroup [70, $88,89]$. Alternatively $S O(10)$ can be broken directly to the SM group, without intermediate steps [90]. All the possible breaking paths from $S O(10)$ can be seen in Figure 3. The particular symmetry breaking scenario that is realized in a $S O(10)$ model depends exclusively on the scalar sector of the theory and the configuration of the vacuum, and it constitutes one of the major differences among $S O(10)$ models in the literature.

Regular $S O(10)$ models may suffer from some of the same issues as regular $S U(5)$, namely rapid proton decay can occur with a low unification scale. The main solution to this problem, as it was with $S U(5)$, is the addition of supersymmetry. SUSY $S O(10)[90,91]$ theories are rather popular and given the large number of degrees of freedom they possess, such as symmetry breaking pattern, field content, etc., they can easily avoid many of the traditional issues in unified theories.

Alike to the $S U(5)$ model, it is possible to construct alternative embeddings of the SM fermions into representations of the group via the addition of an Abelian group. In the flipped $S O(10) \times U(1)$ model [92] the SM fermion content is not fully embedded into the 16 representation of the group, but rather into the direct product $\mathbf{1 6}_{1} \oplus \mathbf{1 0}_{-2} \oplus \mathbf{1}_{4}$. This model loses its unified nature in favor of more degrees of freedom for the Yukawa and symmetry breaking sectors of the theory, which are no longer constrained by the statements above [80].

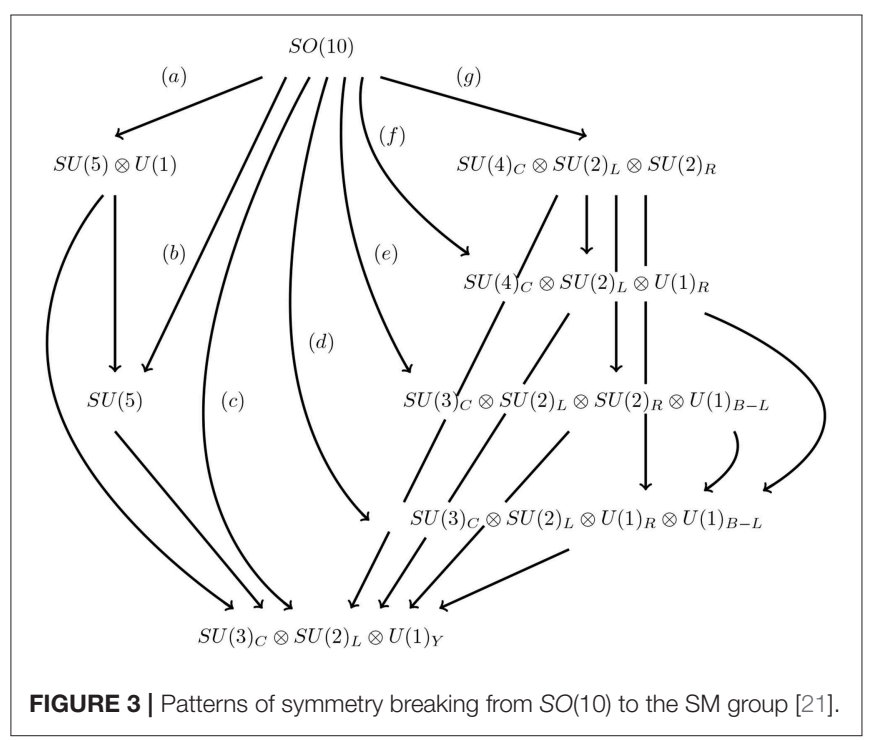




\section{5. $E_{6}$}

The GUT models described so far have unification groups that are part of the infinite series $S U(n)$ or $S O(2 n)$, which means that for each successful model with particular $n$ there is an infinite number of alternatives with order larger than $n$. For instance, the $S O(18)$ group has been studied as a candidate for gauge and family unification [93]. The exceptional algebras, however, are unique so they are more aesthetically appealing candidates as theories of unification [20, 94]. Among all exceptional algebras, only $E_{6}$ is large enough to contain the $\mathrm{SM}$ as a subgroup and admits complex representations.

The fermionic content in the $E_{6}$ theory is embedded in the fundamental 27-dimensional representation of the group, which contains the SM fermions plus exotic fields. The particular allocations of SM fermions into the 27 representation depends on the subgroup of $E_{6}$ that breaks into after SSB, which is typically either the trinification group, $S U(3)_{c} \times S U(3)_{L} \times S U(3)_{R}$ [95-97] or $S O(10) \times U(1)[98,99]$. The decomposition of the fundamental 27 into these subgroups is

$$
\begin{array}{lll}
\mathbf{2 7} \rightarrow\{\mathbf{1}, \mathbf{3}, \mathbf{3}\}+\{\mathbf{3}, \mathbf{3}, \mathbf{1}\}+\{\overline{\mathbf{3}}, \mathbf{1}, \overline{\mathbf{3}}\}, & {\left[S U(3)_{c} \times S U(3)_{L} \times S U(3)_{R}\right]} \\
\mathbf{2 7} \rightarrow \mathbf{1 6}_{1}+\mathbf{1 0}_{-2}+\mathbf{1}_{4}, & {[S O(10) \times U(1)]}
\end{array}
$$

The field content in $E_{6}$ models is quite vast. There are 78 gauge bosons, of which only 45 survive at lower scales if $S O(10)$ is the breaking path, or even fewer in the case of $S U(3) \times S U(3) \times S U(3)$, just 24. The minimal scalar content needs at least a scalar field in the combination $\overline{\mathbf{2 7}}+\overline{\mathbf{3 5 1}}+\overline{\mathbf{3 5 1 ^ { \prime }}}$, which contains the SM Higgs, and a scalar responsible for SSB of $E_{6}$, which is dependent upon the pattern of symmetry breaking, e.g., a $\mathbf{7 8}$ for breaking to $S O(10) \times U(1)$.

One of the main motivations for $E_{6}$ as a unification group comes from superstring theory, where it was shown to emerge as a four-dimensional compactification of the heterotic $E_{8} \times E_{8}$ superstring theory $[24,25]$. In fact, the presence of compactified extra dimensions near the scale of $E_{6}$ breaking can trigger symmetry breaking of the $E_{6}$ group via the Hosotani mechanism [100] straight into $S U(3) \times S U(2) \times U(1) \times$ $U(1) \times U(1)$.

Most of the research in $E_{6}$ theories has been typically considered only within the scope of supersymmetry, which ties in with their motivation as low-energy limits of superstring theory where spacetime supersymmetry emerges naturally after compactification. Being a simple Lie group, $E_{6}$ benefits from the prediction of gauge coupling unification in supersymmetry, as did $S U(5)$ and $S O(10)$, which strengthens the motivation. In addition to the rich phenomenology of the MSSM, the $E_{6}$ model adds quite a few phenomenological predictions on its own, from exotic fermion states to new heavy gauge bosons [101, 102].

\section{SELECTED TOPICS IN GUTS}

Model building in unified theories involves more than the selection of the group theory properties as introduced in section 2. There are a few outstanding issues that need to be addressed in order to construct a realistic model. Gauge coupling unification is typically one of the most pressing issues, which can often be resolved by intermediate steps in the breaking chain or by the addition of supersymmetry to the theory. In this section we describe the interplay between SUSY and GUTs, as well as other topics such as the generation of light neutrino masses and the unification of the Yukawa couplings.

\subsection{Supersymmetry and GUTs}

Supersymmetry is a very appealing theory on its own right. It is one of the most aesthetically pleasing extensions of the Standard Model and it has an extremely rich phenomenology that can be readily tested at colliders and other experiments. SUSY GUTs [22] are a conglomerate of the numerous advantages of unified theories and the predicting power of supersymmetry. One of the most attractive features of SUSY is that it can stabilize the electroweak scale against quantum corrections, the so called hierarchy problem $[103,104]$ and provides a mechanism for dynamic electroweak symmetry breaking $[105,106]$.

In addition, if $R$-parity is conserved [107] the lightest supersymmetric particle (LSP) is stable. Therefore, SUSY automatically predicts the existence of a Dark Matter candidate and can easily produce scenarios with the correct relic abundance [108, 109].

As previously mentioned, one of the major motivations for SUSY GUTs is that the minimal MSSM model predicts gauge coupling unification at some high scale $\sim 10^{16}$ [110]. As was seen in Figure 2, just taking the one-loop RGE flow of the gauge couplings, the unification at the GUT scale is fairly successful. The RGEs for the gauge couplings at one-loop have an analytic solution of the form [111]

$$
\alpha_{i}^{-1}(\mu)=\alpha_{G U T}^{-1}+\frac{b_{i}}{2 \pi} \log \left(\frac{M_{G U T}}{\mu}\right)
$$

where $i=1,2,3$ labels the coupling of the $U(1), S U(2)$, and $S U(3)$ subgroups of the SM gauge group, and $b_{i}$ are parameters that depend on the field content. For the MSSM these are $b_{i}=$ $(33 / 5,1,-3)$. With a degenerate sparticle spectrum at $100 \mathrm{GeV}$, these one-loop RGEs unify at $M_{G U T} \sim 2.5 \times 10^{16} \mathrm{GeV}$ with $\alpha_{G U T} \sim 0.0388$.

This picture, however, relies on a light and almost degenerate supersymmetric spectrum. For heavier or split spectra the situation changes drastically, often spoiling gauge unification altogether. A unification measure can be defined to assess how the unification of gauge couplings changes with the masses of the supersymmetric particles as

$$
\Delta \mu=\frac{\min \left(\mu_{12}, \mu_{23}\right)}{\max \left(\mu_{12}, \mu_{23}\right)},
$$

where $\mu_{i j}$ is the energy scale at which $\alpha_{i}^{-1}$ and $\alpha_{j}^{-1}$ unify. Figure 4 shows how the unification measure varies with respect to the SUSY scale for an MSSM model with degenerate SUSY masses (blue). One can distinctly see that for larger sparticle masses, the unification of gauge couplings significantly worsens, from a 70\% unification for $M_{S U S Y} \sim 100 \mathrm{GeV}$ to $<30 \%$ at $M_{S U S Y} \sim 1 \mathrm{TeV}$. 


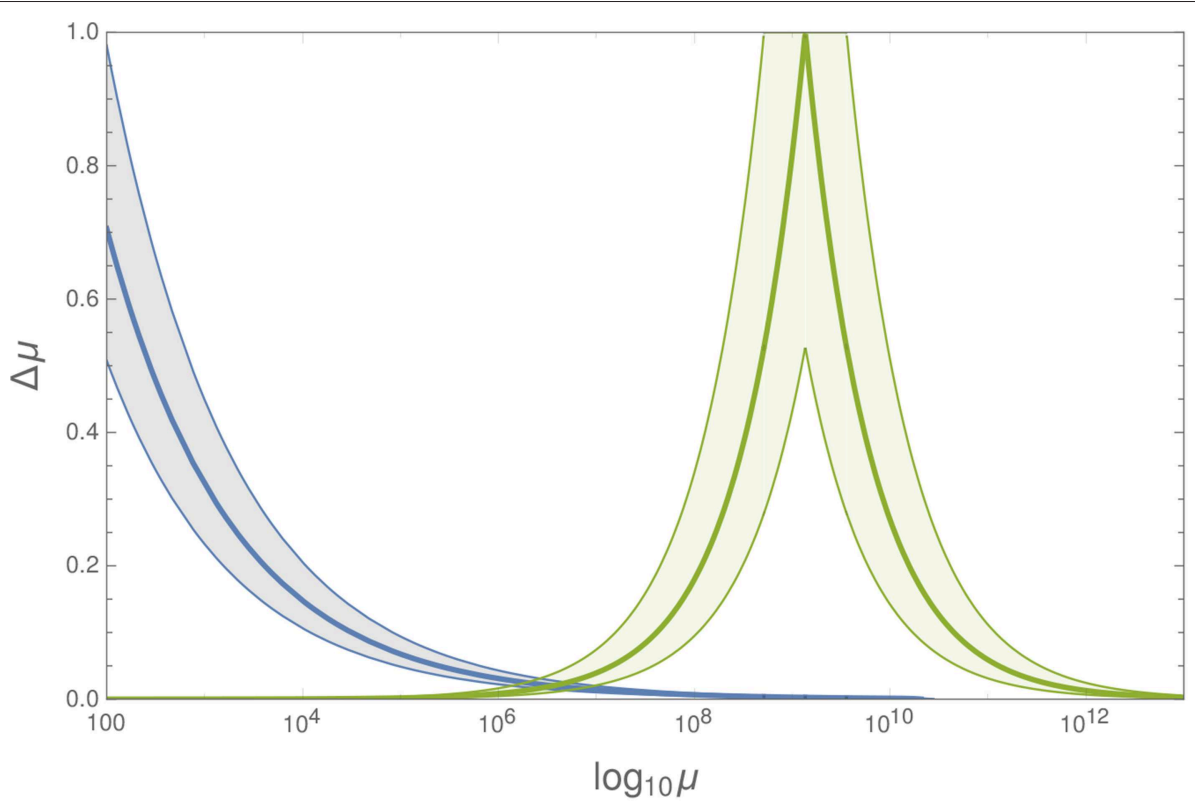

FIGURE 4 | Evolution of the unification measure with the SUSY scale for the MSSM with almost degenerate masses (blue) and scenario with lighter electroweakinos (green), calculated at one-loop. Shaded regions include threshold corrections over the solid lines.

Consequently, in addition to solving the little hierarchy problem without too much fine tuning, a light sparticle spectrum is clearly preferred to achieve gauge coupling unification.

In spite of the appeal of SUSY GUTs, the combined effort of several collider experiments has not found any clear evidences of SUSY particles ${ }^{4}$. Hence, minimal and light versions of the MSSM are in tension with experimental evidence and that makes achieving gauge coupling unification much harder. This tension relaxes slightly once the mass degeneracy condition is forgone. If the sparticle masses vary considerably across the spectrum, it is possible to evade experimental bounds for those masses more strongly constrained (e.g., gluinos, squarks, etc.) while keeping part of the spectrum light. Mass splittings across the supersymmetric spectrum can be taken into account by the contribution of threshold corrections at the SUSY scale, which are of the type $[112,113]$

$$
\begin{aligned}
\lambda_{i}\left(M_{S U S Y}\right)= & \frac{1}{12 \pi}\left(\sum_{\phi} S_{i}(\phi) \log \frac{m_{\phi}}{M_{S U S Y}}\right. \\
& \left.+8 \sum_{\psi} S_{i}(\psi) \log \frac{m_{\psi}}{M_{S U S Y}}\right),
\end{aligned}
$$

with $\phi$ the scalar fields in the MSSM (sfermions), $m_{\phi}$ their masses and $S(\phi)$ their Dynkin indices; and $\psi$ the fermions in the MSSM (gauginos and Higgsinos), $m_{\psi}$ and $S(\psi)$ their masses and Dynkin indices. The shaded blue region in Figure 4 corresponds to MSSM models with slightly non-degenerate masses. Although these models exhibit the same trend as before, decreasing the

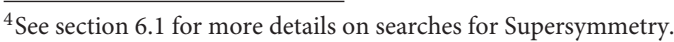

unification measure as $M_{S U S Y}$ increases, some of these achieve a better gauge coupling unification than the degenerate case, with up to $60 \%$ unification for $M_{S U S Y} \sim 1 \mathrm{TeV}$.

Supersymmetric models with large splittings in the particle spectrum can modify this picture significantly. A special case, known as Split Supersymmetry [114, 115], has all the sfermions decoupled at the GUT scale and only gauginos and Higgsinos remain light, protected by chiral symmetry. This model is very well motivated within the context of unified theory, because the decoupled fields form full multiplets of $S U(5)$, so the unification of the gauge couplings is not affected [114]. Hence, the behavior of the unification measure for these models is identical to the semi-degenerate MSSM case from above (blue line and shaded region in Figure 4), but has the advantage of allowing a lighter spectrum since some of the strongest experimental constraints are on the squark masses, which are decoupled from the spectrum. These Split-SUSY models, however, predict the existence of a light gluino, which is unfortunately strongly constrained by experiments. Alternative versions of this model with light electroweakinos $(\sim 100 \mathrm{GeV})$ and heavy gluinos ( $\sim 5 \mathrm{TeV}$ ) have been studied [116], but these fail to provide successful gauge coupling unification for a light spectrum, slowly improving at larger scales, as can be noticed from the green solid line and shaded region (threshold corrections) in Figure 4. SplitSUSY and the light electroweakino model are just two extreme cases, the former requiring very light spectrum for successful unification and the latter a heavy spectrum. A number of models can be constructed with different spectra that have intermediate predictions for gauge coupling unification. In fact, with a precise analyses of threshold corrections, a number of supersymmetric models with large mass splittings have been shown to achieve exact unification, with a relatively light spectrum [117]. 


\subsection{Neutrino Masses}

The observed oscillations of neutrino flavors [10,118] require any successful extension of the SM to incorporate non-zero masses for at least two neutrino species. Effectively, these masses are generated by the 5 -dimensional Weinberg operator

$$
\mathcal{O}_{W}=\frac{c_{W}}{\Lambda} L L H H,
$$

where $c_{W}$ is the Wilson coefficient, $\Lambda$ denotes the operator's cut-off scale and $L$ and $H$ are the lepton and Higgs doublets, respectively. A typical UV-completion of this effective operator is some kind of seesaw mechanism $[66,119]$, which allows to satisfy elegantly the requirement of tiny neutrino mass size. Generally, these neutrino mass schemes assume a presence of new, heavy degrees of freedom, which are ideally motivated by other BSM physics. As has been shown, a number of different seesaw set-ups can be very naturally incorporated within the GUT framework. Provided that only a single type of new particle is added to the SM field content, there are three basic tree-level seesaw types [120].

\subsubsection{Seesaw Type I}

In the original and simplest seesaw mechanism of type I the righthanded neutrino singlets must be added to the model $[66,121-$ 123]. As the current experimental data require only two neutrinos to be massive, the minimal scenario must include two righthanded neutrino states. This extension then allows to write both Dirac and Majorana neutrino mass terms

$$
\mathcal{L}_{N}=-y^{v} \bar{L}^{\ell} \tilde{H} N^{I}-\frac{1}{2}\left[M_{M}\right]_{I J} N^{I^{T}} C N^{J}+\text { h.c. }
$$

where $y^{v}$ is the matrix of neutrino Yukawa couplings and $M_{M}$ denotes the Majorana mass matrix. Hence, taking $m_{D}=y^{v} v$, with $v$ being the SM Higgs vev, the neutrino mass matrix can be written in the usual form

$$
\mathrm{M}=\left(\begin{array}{cc}
0 & m_{D} \\
m_{D}^{T} & M_{M}
\end{array}\right)
$$

The block-diagonalisation of this matrix leads to the light mass of the oscillating neutrinos

$$
M_{v}^{I}=-m_{D} M_{M}^{-1} m_{D}^{T}
$$

as the Majorana mass parameter can be chosen to be arbitrarily large. Considering the neutrino Yukawa couplings of order one and the Majorana mass around $10^{14} \mathrm{GeV}$, the desired neutrino mass sizes of order $m_{v} \approx 0.1 \mathrm{eV}$ are obtained. The type-I seesaw mechanism can be implemented in the GUT framework. Particularly, it arises very naturally in SO(10) GUT, where the right-handed neutrino singlet can be accommodated together with all the other fermions in a single $\mathbf{1 6}_{F}$ spinor representation.

\subsubsection{Seesaw Type II}

The second possibility to construct a seesaw mechanism is to assume a heavy scalar $S U(2)_{L}$-triplet $\Delta_{L}$,

$$
\{\mathbf{1}, \mathbf{3}, 2\} \equiv \Delta_{L}=\Delta_{L} \cdot \boldsymbol{\tau}=\left(\begin{array}{cc}
\frac{1}{\sqrt{2}} \Delta^{+} & \Delta^{++} \\
\Delta^{0} & -\frac{1}{\sqrt{2}} \Delta^{+}
\end{array}\right),
$$

which allows to write the following Lagrangian terms

$$
\begin{array}{r}
\mathcal{L}_{\Delta}=\left[y_{\ell \ell^{\prime}}^{\Delta} L^{\ell T} C\left(i \tau^{2}\right) \Delta_{L} L^{\ell^{\prime}}+\mu H^{T}\left(i \tau^{2}\right) \Delta_{L}^{*} H+\text { h.c. }\right] \\
+M_{\Delta}^{2} \operatorname{Tr}\left[\Delta \Delta^{\dagger}\right] .
\end{array}
$$

Diagonalisation of the type-II seesaw mass matrix [67, 124, 125] then generates neutrino mass

$$
M_{v}^{I I}=\frac{\mu v^{2}}{M_{\Delta}^{2}} y^{\Delta}
$$

and for $M_{\Delta} \gg v$ the required suppression is obtained.

Also this seesaw can be responsible for neutrino mass generation in GUTs. For instance, in $S O(10)$ unification the left-handed scalar triplet is contained by the $\mathbf{1 2 6}$ Higgs field, which is usually considered to be present in the scalar sector of the theory. It has been shown that type-II seesaw can be the dominant neutrino mass scheme within both SUSY [126] and non-SUSY [127] SO(10) GUTs.

\subsubsection{Seesaw Type III}

The third option for a UV-completion of the Weinberg operator in Equation (14) is to introduce new fermionic $S U(2)_{L}$ triplets $\mathbf{T}_{F}^{I}[128]^{5}$. Their interaction with the SM content is analogous to the type I seesaw, namely,

$$
\mathcal{L}_{T_{F}}=y_{\ell J}^{T_{F}} L^{\ell T} C\left(i \tau^{2}\right)\left(\mathbf{T}_{F}^{J} \cdot \boldsymbol{\tau}\right) H+M_{I J}^{T_{F}}\left(\mathbf{T}_{F}^{I}\right)^{T} C \mathbf{T}_{F}^{J}+\text { h.c.. }
$$

The neutrino mass matrix for type III seesaw then reads

$$
M_{v}^{I I I}=\left(y^{T_{F}}\right)^{T} v^{2}\left[M^{T_{F}}\right]^{-1} y^{T_{F}}
$$

and for $M^{T_{F}} \gg y^{T_{F}} v$ the smallness of neutrino masses is ensured.

The incorporation of the type-III seesaw mechanism into GUTs has been studied in literature [39, 120, 129]. When implemented within $S U(5)$ models, type-III seesaw comes automatically in hand with the type-I seesaw, as both fields responsible for these mechanisms share the same adjoint representation $2 \mathbf{4}_{F}$.

\subsubsection{Inverse Seesaw}

At low energies the light neutrino masses can be generated at tree level via the so called inverse seesaw mechanism. This string theory motivated [25] scheme can be constructed when a nonminimal lepton content of a given theory is assumed. Namely, extra singlet leptons must be added to the model, which is in general allowed for any gauge theory [119]. The minimalistic extension of the SM particle content leading to inverse seesaw requires a pair of left-handed two-component lepton singlets $N^{c}$ and $S$ [98]. Taking three generations of these new singlet fields, one can write the $9 \times 9$ mass matrix of the neutral leptons in the basis $\left\{v_{L}^{\ell}, N^{I^{c}}, S^{A}\right\}$ (with $A=a, b, c$ ) as

$$
\mathrm{M}^{\mathrm{IS}}=\left(\begin{array}{ccc}
0 & m_{D} & 0 \\
m_{D}^{T} & 0 & M \\
0 & M^{T} & \mu
\end{array}\right)
$$

\footnotetext{
${ }^{5}$ Similarly to the right-handed neutrino singlets, only two triplets are necessary,
} although three (one per flavor) are considered here. 
where $\mathrm{M}$ and $\mu$ are the mass matrices corresponding to the $S U(2)_{L}$ singlets, while $m_{D}$ is the Dirac neutrino mass matrix as usual. As predicted by some string models, the Majorana mass entries corresponding to $v_{L}$ and $N$ are zero. Thus, the only Majorana mass parameter is the matrix $\mu$, which corresponds to the extra singlet $S$. This entry is then responsible for lepton number violation. If $\mu$ is set to be zero, the $B-L$ symmetry is restored, the matrix $M^{\mathrm{IS}}$ degenerates and the three oscillating neutrinos become massless.

On the other hand, for non-vanishing $\mu$ such that $\mu \ll m_{D} \ll M$ the resulting mass matrix of the light neutrino eigenstates reads

$$
M_{v}^{\mathrm{IS}}=m_{D} M^{-1} \mu\left[M^{T}\right]^{-1} m_{D}^{T}
$$

The main difference from the standard seesaw scenarios is that in the present case neutrinos become light for $\mu \rightarrow 0$, not for large values of Majorana mass parameter. This is also the reason why one talks about "inverse" seesaw. As vanishing $\mu$ enhances the symmetry of the theory, the assumption of its small value can be considered to be natural $[130,131]$.

\subsubsection{Linear Seesaw}

A particularly interesting realization of the inverse seesaw mechanism can be constructed within the SO(10) GUTs framework with broken D-parity [132]. The so called linear seesaw mechanism consists in extending the minimal fermionic content of the $S O(10)$ model, contained by three copies of the $\mathbf{1 6}_{F}$ representation, by three gauge singlets $S^{A}$. The original version of this scheme was designed within the supersymmetric $S O$ (10) framework; however, it can be constructed also in nonsupersymmetric scenarios. The mass matrix for the neutral fermions in the basis $\left\{v_{L}^{\ell}, N^{I^{c}}, S^{A}\right\}$ has the following form

$$
\mathrm{M}^{\mathrm{LS}}=\left(\begin{array}{ccc}
0 & \mathrm{~m}_{D} & \mathrm{~m}_{L} \\
\mathrm{~m}_{D}^{T} & 0 & \mathrm{M} \\
\mathrm{m}_{L}^{T} & \mathrm{M}^{T} & 0
\end{array}\right)
$$

Here, $m_{D}$ denotes the Dirac neutrino mass, $M$ is the heaviest Dirac neutrino mass term mixing $N-S$ and $m_{L}$ stands for the small term mixing $v-S$, which breaks the $(B-L)$ symmetry. The light neutrino masses are then given by the expression

$$
\mathrm{M}_{v}^{\mathrm{LS}} \simeq \mathrm{m}_{D}^{T} \mathrm{M}^{-1} \mathrm{~m}_{L}+\left(\mathrm{M}^{-1} \mathrm{~m}_{L}\right)^{T} \mathrm{~m}_{D},
$$

which depends linearly on $\mathrm{m}_{D}$ (and therefore also on corresponding Yukawa couplings). In the present scenario it is the large parameter $M$ given by the unification scale what ensures the smallness of neutrino masses. Hence, the lightness of neutrinos is independent of the $(B-L)$ symmetry breaking scale, which can consequently lie at low, experimentally accessible energies without spoiling the desired size of neutrino masses or the unification.

\subsubsection{Other Neutrino Mass Models}

Despite the success of seesaw mechanisms, one can think of a number of alternative neutrino mass generation schemes. From the phenomenological point of view, these can be even more interesting, as they often predict (unlike the three usual seesaws) a low-energy origin of neutrino masses. The light neutrino masses are obtained using a small lepton-number-violating parameter (similarly as in the inverse seesaw), or they can be suppressed by loops and small Yukawa couplings. While the former option can be realized e.g., within supersymmetric models with R-parity breaking [133], the latter possibility refers to the models of neutrino mass generation via calculable radiative corrections (i.e., the Zee mechanism) $[41,134]$. A two-loop mechanism generating neutrino masses within a minimal $S O(10)$ GUT was identified by Witten [135] and the same scheme can be constructed also in the flipped SU(5) context [136-138].

\subsection{Yukawa Coupling Unification and Fermion Masses}

In fully unified theories, such as $S U(5)$ and $S O(10)$, the gauge couplings must unify at some high energy scale. This is typically achieved automatically in SUSY GUTs, as mentioned above, due to the RGE flow of the MSSM gauge couplings (c.f. Figure 2), but it can also be achieved through the addition of new scalar representations [139] or with a multi-step symmetry breaking pattern [91].

Along gauge coupling unification, $S U(5)$ and $S O(10)$ models also require the unification of the Yukawa couplings. The largest hierarchy on the fermion masses happens in the third generation where $m_{t} / m_{b} \sim 40$ and $m_{b} / m_{\tau} \sim 2.3$, hence Yukawa unification in GUTs is always studied within the third generation only. In $S U(5)$ the charged leptons live in the same representation as the down-type quarks, $\overline{\mathbf{5}}$, and as such it is expected that at the GUT scale $y_{b}=y_{\tau}$, whereas in $S O(10)$ all SM fermions are embedded into the same 16-dimensional representation, so the unification condition becomes $y_{t}=y_{b}=y_{\tau}$.

Although a natural prediction of GUTs, Yukawa unification is not easily achieved in the vanilla $S U(5)$ and $S O(10)$ models [2931]. As can be seen in the left-side plot of Figure 5, the Yukawa couplings in the SM are far from unification. In spite of this, a few successful attempts to solve the unification of $y_{b}$ and $y_{\tau}$ in $S U(5)$ inspired models have been performed, either by including large scalar $S U(5)$ representations to the field content $[31,139,140]$ or by adding Planck scale suppressed interaction of the Higgs field to the SM fermions [141].

In SUSY GUTs, however, Yukawa unification can often be achieved in some regions of the full supersymmetric parameter space. As can be seen in the right-side picture in Figure 5, the Yukawa couplings in the MSSM tend to run toward convergence at high scales, and they can be seen to almost unify for large values of $\tan \beta$ [142-145]. This occurs because the third generation fermion masses depend on $\tan \beta$ in the following way [146]

$$
m_{t}=\frac{v}{\sqrt{2}} y_{t} \sin \beta, \quad m_{b}=\frac{v}{\sqrt{2}} y_{b} \cos \beta, \quad m_{\tau}=\frac{v}{\sqrt{2}} y_{\tau} \cos \beta,
$$

which can realize the hierarchy $m_{t} \gg m_{b}, m_{\tau}$ even in $S O(10)$ or $E_{6}$ models where one expects $y_{t} \sim y_{b} \sim y_{\tau}$. These solutions with large $\tan \beta$ can spoil radiative EW symmetry breaking in unified models, since $B \mu \simeq \frac{M_{A}^{2}}{\tan \beta} \simeq 0$ implies that $m_{H_{d}}^{2}-m_{H_{u}}^{2}>$ $m_{Z}^{2}$ [147], contrary to the unified picture where $m_{H_{u}}^{2}=m_{H_{d}}^{2}$. This issue can often be resolved either by ad hoc splittings of the Higgs 

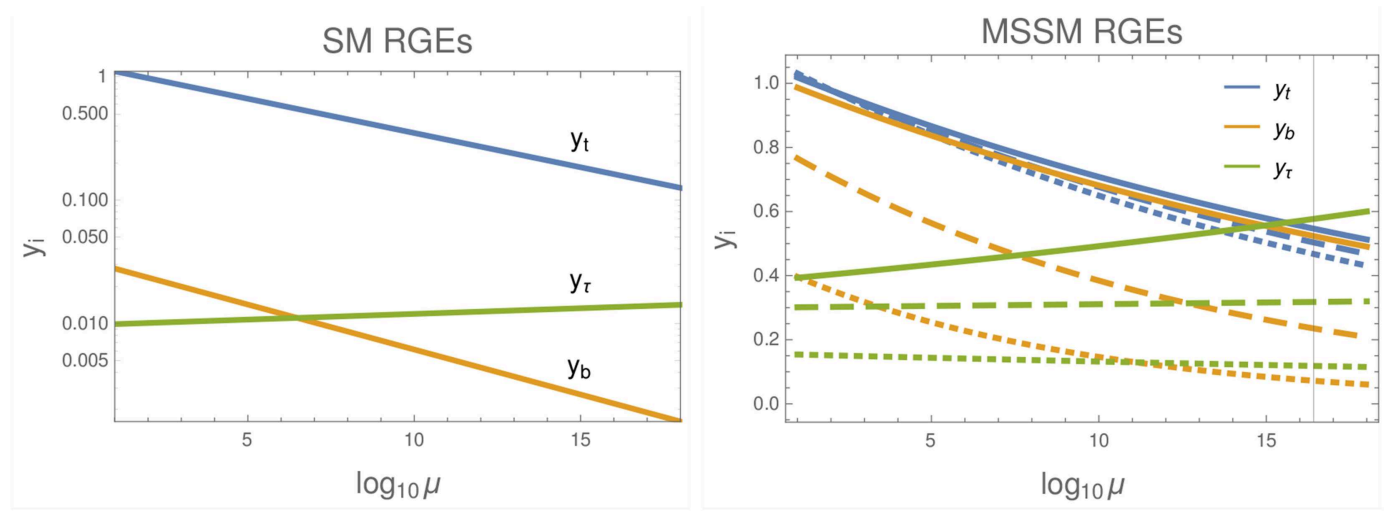

FIGURE 5 | One loop renormalisation group flow of the SM (left) and MSSM (right) Yukawa couplings, with $m_{0}=2$ TeV, $m_{1 / 2}=3$ TeV, $A_{0}=0$ and tan $\beta=40$ (solid), $\tan \beta=30$ (dashed) and $\tan \beta=15$ (dotted).

soft masses at the GUT scale, or by considering the effect of $D$ terms in the boundary conditions at the GUT scale [148], which naturally imposes a splitting of $m_{H_{d}}^{2}-m_{H_{u}}^{2}=4 m_{D}^{2}$.

In addition to satisfying $m_{t} \gg m_{b}, m_{\tau}$ for unified Yukawa couplings, one can lift the hierarchy between $m_{b}$ and $m_{\tau}$ with the inclusion of radiative corrections on the $b$ mass. At one loop the $b$ quark couples to $H_{u}$ via a gluino or Higgsino loop [146], as can be seen in Figure 6, which adds a correction to $m_{b}$ of the type [149]

$$
\delta m_{b} \simeq \frac{v}{\sqrt{2}} y_{b} \frac{\sin \beta}{16 \pi^{2}}\left(\frac{8}{3} g_{3}^{2} \frac{\mu m_{\tilde{g}}}{m_{\tilde{b}^{2}}}+y_{t}^{2} \frac{\mu A_{t}}{m_{\tilde{t}^{2}}}\right) .
$$

Though similar corrections appear for $m_{t}$ and $m_{\tau}$, they are negligible compared to $\delta m_{b}$. The correction on $m_{t}$ is not proportional to $\tan \beta$, which is required to be large to satisfy $t-b-\tau$ unification. Further, $\delta m_{\tau}$ does not have a gluino loop and the Higgsino contribution is inversely proportional to $m_{\tilde{v}_{t}}$ which is typically much larger than $m_{\tilde{t}}$, and therefore the contribution is small. These radiative corrections on $m_{b}$ are proportional to $\tan \beta$ and therefore can be significant, up to 50\% [148], which can spoil the hierarchy $m_{t} \gg m_{b}$. There are regions of the SUSY parameter space, however, where it is possible to reduce $\delta m_{b}$ while keeping $\tan \beta$ large [148-150], thereby successfully predicting $t-b-\tau$ unification, even factoring in LHC searches [151].

In SUSY SU(5) models the more straightforward boundary condition $y_{b}=y_{\tau}$ is imposed. It was found that, in addition to the large $\tan \beta$ scenarios from above, $b-\tau$ unification can also be achieved in a region of parameter space with low $\tan \beta[152,153]$. However, a sufficiently low $\tan \beta$ might struggle to lift sufficiently the tree level Higgs mass to the observed value, and hence there remains some tension between unified $b-\tau$ models of low $\tan \beta$ and the observed Higgs mass [154].

A number of other mechanisms have been proposed to satisfy the Yukawa unification conditions. Intermediate breaking steps, such as the Pati-Salam group, can modify the Yukawas RGEs in a favorable manner achieving quasi-unification [155, 156]. Non-canonical seesaw mechanisms in neutrino models require $b-\tau$ unification to match the observed neutrino mixings [157].
Or the inclusion of certain higher dimensional operators can successfully yield Yukawa unification [158].

Beyond the unification of the Yukawa couplings for each of the families of SM fermions, the mass hierarchies among the different families remains an open question. Although GUTs by themselves do not make predictions on the nature of this hierarchy, they often include a fair amount of parameters and mixing matrices that are unconstrained and can fit the fermion masses. Additionally GUTs are often extended with family symmetries, continuous or discrete, which can, with a smaller set of parameters, accurately predict the fermion mass hierarchies, as well as their mixings and CP phases encoded in the CKM and UPMNS matrices [159-163]. We will not discuss family symmetries any further since they fall beyond the scope of this work.

\section{MODERN DAY GUTS}

Since their first appearance in the late 70 s, a large number of GUT models have been proposed. These vary according to the symmetry group employed, the symmetry breaking mechanism and field content among others. Some of them were driven by the experimental results of the time and other by new theoretical insights. In this section we attempt to outline a small, nonexhaustive, subset of GUT models, aiming to explore those with strong phenomenological consequences and some that have been in the spotlight in recent years. We thus focus on left-right symmetric models, SUSY SO(10), trinification models and $\mathrm{E}_{6} \mathrm{SSM}$.

\subsection{Left-Right Symmetric Models}

One of the minimal extensions of the SM is the earlier mentioned left-right symmetric model [16, 17, 62, 63, 67]. Despite not being real GUTs, LR models can very conveniently play the role of an intermediate symmetry restored between the electroweak scale and the GUT scale $[69,164,165]$. The LR framework has attracted a lot of attention particularly in connection with the LHC [166178], as it typically predicts new physics at energies that can be probed by the collider searches. 

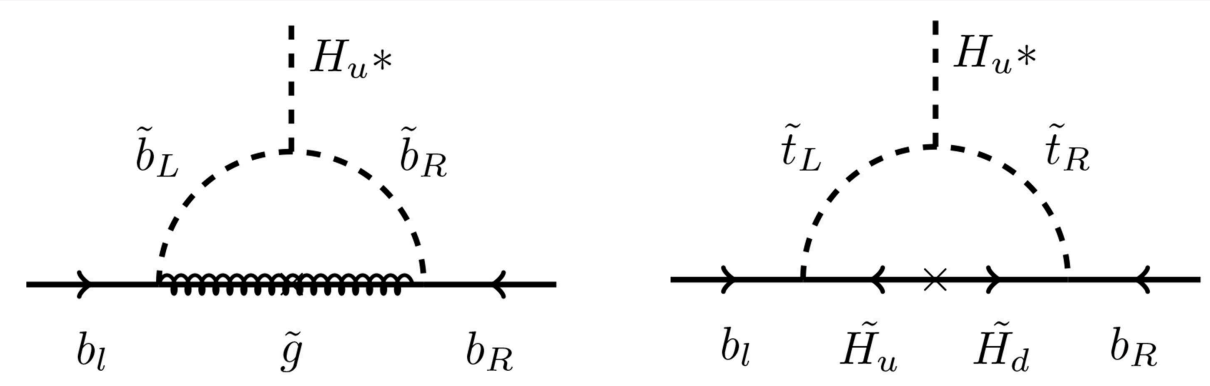

FIGURE 6 | One-loop radiative corrections to $m_{b}$.

The fermionic particle content of LR models is given by a straightforward LR symmetric extension of the SM content, i.e., the right-handed doublets are introduced

$$
\begin{aligned}
& L_{R}^{\ell}=\left(\begin{array}{c}
N^{\ell} \\
\ell_{R}
\end{array}\right) \leftrightarrow\left(\begin{array}{c}
v_{L}^{\ell} \\
\ell_{L}
\end{array}\right)=L^{\ell}, \\
& Q_{R}^{i}=\left(\begin{array}{c}
u_{R}^{i} \\
d_{R}^{i}
\end{array}\right) \leftrightarrow\left(\begin{array}{c}
u_{L}^{i} \\
d_{L}^{i}
\end{array}\right)=Q^{i} .
\end{aligned}
$$

As a result, right-handed neutrinos are naturally included making the left-handed neutrinos acquire mass in the LR models, which is a highly desirable feature of a BSM model. The presence of the right-handed neutrino partners is also essential for cancellation of the $B-L$ gauge anomaly.

The Higgs sector of LR symmetric theories can vary. The minimal scenarios mostly include a scalar bi-doublet ${ }^{6}$

$$
\Phi \equiv\{\mathbf{1}, \mathbf{2}, \mathbf{2}, 0\}=\left(\begin{array}{cc}
\phi_{1}^{0} & \phi_{2}^{+} \\
\phi_{1}^{-} & \phi_{2}^{0}
\end{array}\right),
$$

containing the SM Higgs, which subsequently gives masses to quarks and leptons. The corresponding vev reads

$$
\langle\Phi\rangle=\left(\begin{array}{cc}
v_{\Phi 1} & 0 \\
0 & v_{\Phi 2}
\end{array}\right),
$$

where $v \equiv \sqrt{v_{1}^{2}+v_{2}^{2}}$ and it mixes the left-handed and righthanded gauge bosons as described below.

Besides the bi-doublet, typically a pair of scalar triplets

$$
\Delta_{L} \equiv\{1,3,1,-2\}, \quad \Delta_{R} \equiv\{1,1,3,-2\},
$$

or doublets

$$
\chi_{L} \equiv\{1,2,1,-1\}, \quad \chi_{R} \equiv\{1,1,2,-1\}
$$

must be added to the Higgs sector in order to break the LR gauge group to the SM. In fact, the right-handed scalar is enough to do so, but inclusion of the left-handed triplet (or doublet) preserves

${ }^{6}$ Here, the representations are labeled the usual way in the order $\left\{S U(3)_{C}, S U(2)_{L}, S U(2)_{R}, U(1)_{B-L}\right\}$. the LR symmetry (so called "manifest LR symmetry"), i.e., the $S U(2)_{L}$ and $S U(2)_{R}$ gauge couplings are equal: $g_{L}=g_{R}$.

If no additional fermions besides the SM fermionic content are considered, at least two bi-doublets must be present in the scalar sector to account for the correct SM flavor physics [164]. In a model with a single bi-doublet the Yukawa Lagrangian implies that the up-quark mass matrix is proportional to the down-quark mass matrix (independently of the vev structure); thus, the CKM matrix becomes trivial $V_{\mathrm{CKM}}=1$.

Consequently, the LR symmetry breaking takes place in two steps. At first, the neutral component of right-handed scalar triplet (or doublet) gets the vev $v_{R}$ and breaks the LR gauge group to the SM gauge group. Subsequently, the bi-doublet acquiring its vev breaks the $S M$ gauge group to $S U(3)_{C} \otimes U(1)_{Q}$. Based on the observations it can be assumed that $v_{R} \gg v_{\Phi 1}, v_{\Phi 2}$.

Depending on the scalar content of a particular LR model, different ways of light neutrino mass generation can be employed. Having right-handed neutrino singlets means that type-I seesaw is always the option. In general, the neutrino mass matrix can take the form

$$
\mathrm{M}_{v}=\left(\begin{array}{cc}
\mathrm{M}_{M, L} & \mathrm{~m}_{D} \\
\mathrm{~m}_{D}^{T} & \mathrm{M}_{M, R}
\end{array}\right),
$$

where $\mathrm{m}_{D}$ denotes the Dirac mass matrix, while $\mathrm{M}_{M, L}$ and $\mathrm{M}_{M, R}$ are the Majorana mass matrices corresponding to the left-handed and right-handed neutrinos, respectively.

The Yukawa couplings in LR models include the scalar bi-doublet,

$$
\mathcal{L}_{\text {Yukawa }}^{\Phi}=\mathrm{y}_{\ell \ell^{\prime}}^{\Phi} L^{\ell^{T}} C \Phi L_{R}^{\ell^{\prime}}+\tilde{\mathrm{y}}_{\ell \ell^{\prime}}^{\Phi} L^{\ell^{T}} C \tilde{\Phi} L_{R}^{\ell^{\prime}}+\text { h.c., }
$$

where $\tilde{\Phi}=\sigma^{2} \Phi^{*} \sigma^{2}$. Then the Dirac neutrino mass matrix and the mass matrix of charged leptons are in the broken phase given by

$$
\begin{aligned}
\mathrm{m}_{D} & =\mathrm{y}^{\Phi} v_{\Phi 1}+\tilde{\mathrm{y}}^{\Phi} v_{\Phi 2}, \\
\mathrm{~m}_{\ell} & =\mathrm{y}^{\Phi} v_{\Phi 2}+\tilde{\mathrm{y}}^{\Phi} v_{\Phi 1} .
\end{aligned}
$$

In case that the right-handed scalar triplet $\Delta_{R}$ is responsible for the LR symmetry breaking, one can write also the Yukawa couplings for the right-handed lepton doublet in the form

$$
\mathcal{L}_{\text {Yukawa }}^{\Delta_{R}}=\frac{1}{2} \mathrm{y}_{\ell \ell^{\prime}}^{\Delta_{R}}\left(L_{R}^{\ell}\right)^{T} C\left(i \tau^{2}\right) \Delta_{R} L_{R}^{\ell^{\prime}}+\text { h.c., }
$$


where $\Delta_{R}=\Delta_{R} \cdot \tau$. After the triplet acquires its vev

$$
\left\langle\Delta_{R}\right\rangle=\left(\begin{array}{cc}
0 & 0 \\
v_{R} & 0
\end{array}\right),
$$

the LR symmetry is broken and the right-handed neutrino receives Majorana mass $\mathrm{M}_{M, R}=\mathrm{y}^{\Delta_{R}} v_{R} \gg v$, which allows for type-I seesaw mechanism.

When the Higgs sector contains also the left-handed scalar triplet $\Delta_{L}$ with vev

$$
\left\langle\Delta_{L}\right\rangle=\left(\begin{array}{cc}
0 & 0 \\
v_{L} & 0
\end{array}\right),
$$

it generates the left-handed Majorana mass matrix $\mathrm{M}_{M, L}=\mathrm{y}^{{ }_{L}} v_{L}$ switching on type-II seesaw mechanism.

In principle, the type-I and type-II seesaws can be combined giving the "full" seesaw matrix (35). The resulting light neutrino mass matrix reads

$$
\mathrm{M}_{v}^{\mathrm{I}+\mathrm{II}}=\mathrm{M}_{M, L}-\mathrm{m}_{D}\left[\mathrm{M}_{M, R}\right]^{-1} \mathrm{~m}_{D}^{T} .
$$

Specifically, if $v_{\Phi 2}=0$ is assumed for simplicity, then the formula (42) can be rewritten in terms of the parameters of the LR models as

$$
\mathrm{M}_{v}^{\mathrm{LR}}=\mathrm{y}^{\Delta_{L}} v_{L}-\frac{v_{\Phi 1}^{2}}{v_{R}} \mathrm{y}^{\Phi}\left[\mathrm{y}^{\Delta_{R}}\right]^{-1} \mathrm{y}^{\Phi T} .
$$

Hence, if the hierarchy $v_{R} \gg v_{\Phi 1} \gg v_{L}$ is satisfied, the neutrino masses become small.

In models with the LR symmetry breaking driven by the righthanded doublet $\chi_{R}$ instead of the triplet $\Delta_{R}$ the light neutrino masses can be obtained employing the inverse [98, 179-181] and/or linear $[175,182,183]$ seesaw mechanisms, provided that a singlet fermion $\{\mathbf{1}, \mathbf{1}, \mathbf{1}, 0\}$ is added to the model particle content. Alternatively, it is also possible to construct the type-III seesaw mechanism, if a left-handed or right-handed fermionic triplet is present within the LR model [184, 185]. Lastly, neutrino mass generation in LR models via the Zee mechanism can be achieved with the addition of a charged scalar boson $\{\mathbf{1}, \mathbf{1}, \mathbf{1}, 2\}$ [186].

\subsection{SUSY SO(10) Models}

Supersymmetric $S O(10)$ models are rather appealing GUTs, for they combine together the advantages of SUSY, Pati-Salam and $S U(5)$ models. As briefly outlined in section 2.4, $S O(10)$ models unify all fermions of a generation in the SM into a single representation, of dimension $\mathbf{1 6}$. This decomposes into the maximal subgroups as

$$
\begin{aligned}
& \mathbf{1 6} \rightarrow\{\mathbf{4}, \mathbf{2}, \mathbf{1}\}+\{\overline{\mathbf{4}}, \mathbf{1}, \mathbf{2}\},\left[S U(4)_{c} \times S U(2)_{L} \times S U(2)_{R}\right] \\
& \mathbf{1 6} \rightarrow \mathbf{1 0}_{-1}+\overline{\mathbf{5}}_{3}+\mathbf{1}_{-5}, \quad[S U(5) \times U(1)] .
\end{aligned}
$$

As mentioned before, at the renormalizable level the Yukawa sector of $S O(10)$ includes the Higgs representations 10, 120, and $\overline{\mathbf{1 2 6}}$, which are promoted to superfields in SUSY $S O(10)$. Hence, the superpotential of the Yukawa sector is

$$
W_{Y}=\mathbf{1 6}^{T}\left(\mathrm{Y}_{10} \mathbf{1 0}+\mathrm{Y}_{120} \mathbf{1 2 0}+\mathrm{Y}_{126} \overline{\mathbf{1 2 6}}\right) \mathbf{1 6}
$$

where $Y_{i}$ are matrices of Yukawa couplings in family space. One of the most remarkable features of SUSY $S O(10)$ is that, starting from a Yukawa unified scenario, $Y_{10}$ and $Y_{126}$ are sufficient to reproduce the full mass spectrum of SM fermions, along with the measured values of mixings in the quark and neutrino sector [187-197]. The mass matrices of SM fermions $\mathbf{M}_{i}$ can be written as [86]

$$
\begin{aligned}
\mathrm{M}_{d} & =v_{10}^{d} \mathrm{Y}_{10}+v_{126}^{d} \mathrm{Y}_{126} \\
\mathrm{M}_{u} & =v_{10}^{u} \mathrm{Y}_{10}+v_{126}^{u} \mathrm{Y}_{126} \\
\mathrm{M}_{l} & =v_{10}^{d} \mathrm{Y}_{10}-3 v_{126}^{d} \mathrm{Y}_{126} \\
\mathrm{M}_{D} & =v_{10}^{u} \mathrm{Y}_{10}-3 v_{126}^{u} \mathrm{Y}_{126} \\
\mathrm{M}_{L} & =v_{L} \mathrm{Y}_{126} \\
\mathrm{M}_{R} & =v_{R} \mathrm{Y}_{126}
\end{aligned}
$$

where $M_{D}, M_{L}$, and $M_{R}$ are the Dirac and Majorana masses in types I and II seesaw (c.f. section 3.2), and the $v$ 's are the various vacuum expectation values of $\mathbf{1 0}, \overline{\mathbf{1 2 6}}$ and the left and right-handed $S U(2)$ triplets.

The minimal SUSY SO(10) model therefore contains the Higgs superfields $\mathbf{1 0}$ and $\overline{\mathbf{1 2 6}}$, responsible for fermion masses, and a pair of representations $\mathbf{1 2 6}$ and $\mathbf{2 1 0}$ which trigger the symmetry breaking of SO(10) [198-200]. Although quite appealing due to its minimal set of model parameters, this model does not achieve the right level of gauge coupling unification and suffers from rapid proton decay $[87,201]$.

Many solutions have been implemented to resurrect minimal $S O$ (10) models. The spectrum of soft masses in the low energy MSSM strongly affects the outcome of gauge coupling unification, as was seen in section 3.1, hence modifications on the hierarchy of soft masses can help toward solving the issues with SUSY SO(10) models [202-206]. Additionally, extended scalar sectors, either containing a $\mathbf{1 2 0}$ [207, 208] or a $\mathbf{5 4}$ [209] representation, can increase the unification scale through strong threshold effects, thereby alleviating the constraint of nucleon decay. Recently it has been shown that a combination of new Higgs representations with a modified spectrum of soft masses can accommodate gauge coupling unification and nucleon decay constraints, while still being able to predict a suitable spectrum of fermion masses [210].

As with many GUT models, SUSY $S O(10)$ makes predictions that can be tested in a number of different fronts. Collider searches at the LHC [211, 212] as well as dark matter searches [213] can discover the predicted light, TeV-scale, states. Precision tests such as nucleon decays [214, 215], lepton flavor violation [216] and flavor observables [217] can probe the validity of the models at high scales. For more details on probing SUSY $S O(10)$ and GUTs in general see sections 5-7.

\subsection{Trinification}

As a maximal subgroup of $E_{6}$, the trinification gauge group $S U(3)_{c} \times S U(3)_{L} \times S U(3)_{R}$ is an alternative approach to SUSY $S O(10)$ on the road to $E_{6}$ unification. The matter content of 
trinification models per generation typically looks like [218]

$$
\begin{array}{r}
\{\mathbf{1}, \mathbf{3}, \overline{\mathbf{3}}\}=\left(\begin{array}{lll}
h_{11} & h_{22} & e \\
h_{21} & h_{22} & v \\
e^{c} & v^{c} & \phi
\end{array}\right), \quad\{\mathbf{3}, \overline{\mathbf{3}}, \mathbf{1}\}=\left(\begin{array}{lll}
u & d & D
\end{array}\right), \\
\{\overline{\mathbf{3}}, \mathbf{3}, \mathbf{1}\}=\left(\begin{array}{lll}
u^{c} & d^{c} & D^{c}
\end{array}\right)
\end{array}
$$

where $h_{i j}$ are the components of two Higgs doublets, $\phi$ a SM singlet field and $D$ and $D^{c}$ color-triplets. An additional $Z_{3}$ symmetry is often considered to make the gauge couplings unify at the GUT scale, $g_{c}=g_{L}=g_{R}$.

Given the presence of exotic fields in the matter multiplets, trinification models struggle to trigger spontaneous symmetry breaking without making the matter content impossibly heavy. Additional Higgs multiplets [219-222] and/or nonrenormalizable operators [223-226] are usually introduced to alleviate this issue. Unfortunately these models tend to produce tension with current limits on proton decay [227] and collider searches [228, 229].

The fundamental challenge behind the issues of trinification is the complicated vacuum structures and the large number of parameters they depend on. Solutions to this problem involve the addition of family symmetries which reduce the number of parameters and facilitate the study of the symmetry breaking vacuum [230], further simplified by embedding the theory into larger dimensional groups such as $E_{8}[231,232]$.

\section{4. $\mathrm{E}_{6} \mathrm{SSM}$}

The Exceptional Supersymmetric Standard Model (ESSM or $\left.\mathrm{E}_{6} \mathrm{SSM}\right)[233,234]$ is an extension of the MSSM motivated as a low energy effective theory from a $E_{6}$ unified GUT model at high scales. At low scales it has the gauge group $S U(3)_{c} \times S U(2)_{L} \times$ $U(1)_{Y} \times U(1)_{N}$, where the additional $U(1)_{N}$ factor is leftover from the symmetry breaking of $E_{6}$. All the superfields in the theory are contained in three copies of the 27 representation of $E_{6}$, which decompose under the $S U(5) \times U(1)_{N}$ subgroup as [235]

$$
27^{i} \rightarrow \mathbf{1 0}_{1}^{i}+\overline{5}_{2}^{i}+\overline{5}_{-3}^{i}+5_{-2}^{i}+\mathbf{1}_{5}^{i}+\mathbf{1}_{0}^{i}
$$

where $10_{1}^{i}$ and $\overline{\mathbf{5}}_{2}^{i}$ are the matter multiplets for all three generations, with the standard embeddings of matter fields in $S U$ (5) (c.f. section 2.1). The superfields $\overline{\mathbf{5}}_{-3}^{i}$ and $\mathbf{5}_{-2}^{i}$ contain the two Higgs doublets of the MSSM, $H_{u}$ and $H_{d}$, plus two copies of pairs of exotic doublets, $H_{u}^{1,2}$ and $H_{d}^{1,2}$ and three copies of exotic triplets $D_{i}$ and $\bar{D}_{i}$. Lastly, the singlets $\mathbf{1}_{5}^{i}$ and $\mathbf{1}_{0}^{i}$ correspond to exotic singlet fields $S_{i}$, responsible for $U(1)_{N}$ breaking at low scales, and right-handed neutrino fields $N_{i}$, respectively.

Anomaly cancellation of the $U(1)_{N}$ factor in the $\mathrm{E}_{6}$ SSM model is guaranteed so long as the only decoupled state is the singlet neutrino field. $N_{i}$ can be as large as necessary to provide light neutrino masses through type I seesaw mechanism and generate the baryon assymmetry of the Universe via leptogenesis [236, 237]. The remaning fields of the $27^{i}$ multiplets charged under $U(1)_{N}$ remain at energies below the breaking of $U(1)_{N}$ and hence anomalies cancel. Light colored states have dangerous consequences, however, for they can mediate baryon and lepton number violating interactions leading to rapid proton decay. In order to avoid that, the original $\mathrm{E}_{6} \mathrm{SSM}$ model postulates the existence of an approximate $Z_{2}$ symmetry that forbids those interactions. An exact $Z_{2}$ symmetry can also be considered [238, 239], but in such a case additional exotic states must be introduced to ensure that the exotic quarks are not stable.

Gauge coupling unification in the $\mathrm{E}_{6} \mathrm{SSM}$ model requires the addition of incomplete multiplets of $E_{6}$ at low scales, since full multiplets do not modify the unification properties of the RGE flow. A pair of fields $H$ and $\bar{H}$ in conjugate representations are added, to ensure no anomalies are reintroduced. Alternatively, a Pati-Salam intermediate step has been postulated that achieves gauge coupling unification without the need of additional superfields. This "minimal" $\mathrm{E}_{6} \mathrm{SSM}$ model, however, predicts unification at the Planck scale so quantum gravity corrections may play a role and affect the outcome of unification [240].

The $\mathrm{E}_{6} \mathrm{SSM}$ has a rather rich phenomenology since most of the predicted exotic states live at low energies. The constrained $\mathrm{E}_{6} \mathrm{SSM}\left(\mathrm{cE}_{6} \mathrm{SSM}\right)$ is a version of the full $\mathrm{E}_{6} \mathrm{SSM}$ that exploits the properties of unification of $E_{6}$ and assumes universal scalar and gaugino soft masses at the GUT scale [241, 242]. Predictions of the $\mathrm{cE}_{6} \mathrm{SSM}$ include contributions to the Higgs mass and rare decays $[243,244]$ together with light exotic states, such as the $Z_{N}^{\prime}$ associated with the $U(1)_{N}$ broken symmetry, and the color triplet fermions $D$ and $\bar{D}$, all of which can be probed at the LHC [245]. Lastly, as in the MSSM the lightest supersymmetric particle is stable, so it is a valid candidate for dark matter. In contrast with the regular neutralino dark matter in the MSSM, the dark matter candidate in the $\mathrm{E}_{6} \mathrm{SSM}$ is a mixture of binos, winos and higgsinos, as well as the inert singlinos and higgsinos in $H_{u, d}^{i}$ and $S_{i}$ [246-250].

\section{COSMOLOGY AND THE EARLY UNIVERSE}

\subsection{Inflation and GUTs}

Cosmic inflation plays an important role in theories of Grand Unification, as it is needed to dilute relics such as magnetic monopoles, which are produced ubiquitously in GUT models ${ }^{7}$. The requirement to dilute these relics therefore determines the scale of inflation in specific models [251]. Moreover, since generic inflation models are associated with a scale $\Lambda_{\text {inflation }} \sim 10^{16} \mathrm{GeV}$, it becomes attractive to associate the inflaton with a GUT scalar.

To agree with observations, inflation models need to predict a large number of observable e-folds $\left(N=\int_{t_{0}}^{t_{e}} H d t \gtrsim 60\right)$, as well as small spectrum density of fluctuations $\delta \rho / \rho \sim 10^{-5}$. For an effectively single field model, this can be illustrated by the tension between the Lyth bound (a measure of the field excursion necessary to solve the problems inflation was invented to solve) given in terms of the number of e-foldings $N$ [252],

$$
\Delta \phi \sim\left(\frac{r}{0.002}\right)^{1 / 2}\left(\frac{N}{60}\right) M_{p}
$$

${ }^{7}$ However, there are exceptions, most notably the flipped SU(5) SUSY GUT theories. 
and the amplitude of the Cosmic Microwave Background (CMB) anisotropies, which implies [253, 254],

$$
\Lambda_{\text {inf }}^{4}=\left(2.2 \times 10^{16} \mathrm{GeV}\right)^{4}\left(\frac{r}{0.2}\right) .
$$

It is clear, then, that inflation requires a very flat scalar potential. Hence, it is attractive to consider inflation models in which the potential is dynamically generated [255-258].

A successful example of such a model was realized as early as 1983 [259]. This paper considered a potential of a ColemanWeinberg form [255],

$$
V(\phi)=A \phi^{4}\left(\log \frac{\phi^{2}}{v_{\phi}^{2}}-\frac{1}{4}\right)+C
$$

Such a potential can only be made compatible with CMB constraints if $A$ is very small (presently, $A \lesssim 10^{-14}$ [260]). Therefore, the potential in Equation (51) cannot be due to loops of SU(5) gauge bosons. Instead, [259] considered a model in which the inflaton transformed as a singlet of $S U(5)$, couples weakly to the adjoint and fundamental Higgs fields, and therefore obtains a vacuum expectation value when $S U(5)$ breaks into the SM. The original CW-GUT inflation model [259] predicts primordial gravitational waves with tensor-toscalar index $0.02 \leq r \leq 0.1$ [261]. Although this is currently not in tension with the CMB-constraints [253, 254], several modifications have been proposed which predict smaller $r$ [260, 262-264].

An alternative class of GUT inflation models are based on no-scale supergravity. It was realized in 2013 [265] that particular realizations of no-scale supergravity (SUGRA) models of inflation can be equivalent to the Starobinsky model [266], in which inflation is realized from a non-minimal Einstein-Hilbert action $S=\frac{1}{2} \int d x \sqrt{-g}\left(R+R^{2} / 6 M^{2}\right)$. The correspondence can be seen by a conformal transformation, such that the model is equivalent to canonical gravity plus a scalar field [265, 267]. The scalar potential then becomes

$$
V(\phi)=\frac{3}{4} M^{2}\left(1-e^{-\sqrt{2 / 3} \phi}\right)^{2} .
$$

Starobinsky-like models are attractive candidates for inflation models, as they make viable predictions for inflationary observables without the need to introduce a large set of finely tuned parameters. Examples of no-scale SUGRA inflation models include sneutrino inflation, which can be consistently implemented in flipped SU(5) SUSY GUTs [268-272]. Other no-scale GUT inflation models identify the inflaton with the Higgs boson, and circumvent the problems associated with conventional Higgs inflation [273, 274].

A phenomenological approach was taken by Hertzberg and Wilczek [275]. Here it was assumed that inflation is driven by the vacuum energy associated with unification. It was shown that several examples of large-field $\left(\Delta \phi \sim M_{p}\right.$ ) models of inflation give predictions consistent with the CMB-constraints [253, 254].

GUT-inflation has also been studied in combination with other cosmological scenarios, most importantly with nonthermal leptogenesis and the seesaw mechanism for neutrino masses [276-278] ${ }^{8}$. Models of sneutrino inflation are well suited for this purpose [268-272].

\subsection{Cosmological Constraints on Cosmic Strings}

Cosmic strings are generic cosmological predictions of many GUT theories [279-281]. Field theories with broken gauge symmetries may have a vacuum state that is not unique, such that different points in physical space may have distinct (but degenerate) vacuum configurations. By continuity of the field, the interpolating field values must be taken on in between these points, which gives rise to an energetic object called a topological defect, or (in the one-dimensional case) a string.

The simplest description of cosmic strings after their formation approximates the fundamental Nambu-Goto strings. Nambu-Goto strings are characterized by the dimensionless string tension $G \mu$, where $\mu$ is the mass per unit length and $G$ is Newton's constant. Strings produced at the GUT scale typically have a mass per unit length of the order of $\mu \sim 10^{21} \mathrm{~kg} \mathrm{~m}^{-1}$ and a thickness of $10^{-24} \mathrm{~m}$, such that the tension is $G \mu \sim 10^{-6}$ [282]. For comparison, strings formed around the electroweak scale are expected to have much smaller tensions, $G \mu \sim 10^{-34}$. As the expansion of the Universe stretches strings, while the string tension stays constant and in the absence of a decay mechanism, $\rho_{\text {strings }}$ would grow with the scale factor and eventually dominate the energy density of the Universe. Cosmic strings can decay into gravitational or scalar radiation, however. In the presence of such a decay channel an attractor scaling solution is reached, in which the strings maintain a constant fraction of the energy budget.

Cosmic strings could be detected through gravitational lensing and anisotropies in the $\mathrm{CMB}[283,284]$. Cosmic strings imprint on the $\mathrm{CMB}$ as line-like discontinuities, caused by a boost of photons toward the observer as a string moves across the line of sight $[285,286]$. Planck data constrains the Nambu-Goto string tension $G \mu<1.8 \times 10^{-7}$ [287], the non-local string tension $G \mu<10.6 \times 10^{-7}$ [287] and the Abelian Higgs string model $G \mu<2.0 \times 10^{-7}$ [288].

If the strings decay gravitationally, such radiation can be observed in Gravitational Wave (GW) experiments [289-293]. Strong gravitational radiation bursts may be produced by cusps [294-298]: the LIGO/VIRGO collaboration reported an experimental upper limit of $G \mu<10^{-8}$ in some regions of the parameter space, in which gravitational backreaction determines the size of the loops [299]. Pulsar Timing Arrays (PTAs) potentially give more stringent bounds, as they can already probe the stochastic GW background; depending on the model, $G \mu<\mathcal{O}\left(10^{-12}-10^{-11}\right)[300-302]$. However, the relative importance of the gravitational decay channel has been the source of some disagreement in the literature. Simulations of NambuGoto strings [303-308] and full field-theoretic simulations of the Abelian Higgs model [309-311] differ in the typical scale on which the strings form loops. Loops of the order of the string width $r_{s}$ can radiate heavy particles (as the natural mass of coupled particles is $m \sim r_{s}^{-1}$ ); loops of the typical interstring spacing $\xi$ are expected to decay gravitationally [312].

${ }^{8} \mathrm{We}$ expand more on the topic of the baryon assymmetry in subsection 5.4. 
Recent field-theory simulations [311] suggest a mechanism to transport energy from large to small scales, which is not currently understood. Numerical results [313-315] also indicate that the simplest analytical models are due an update, when non-Abelian gauge groups are considered. Furthermore, different groups simulating Nambu-Goto strings differ in the distribution of the loop size. Simulations in which the gravitational radiation backreacts on the string have smoother features, which hinders the formation of smaller loops [303-305]. In these simulations, the PTA constraints on the stochastic background and LIGO/VIRGO constraints on burst are stronger [301].

The shape of the fractional energy density $\Omega_{G W} \equiv$ $f / \rho_{c}\left(d \rho_{\mathrm{GW}} / d f\right)$ power spectrum from cosmic strings is expected to be nearly scale-invariant, with an amplitude defined by the characteristic string tension $G \mu$, such that if it has a large enough amplitude, the signal would be seen in frequency windows of different experiments [316]. This distinguishes the power spectrum from other sources. In particular, an observation of GW at pulsar timing arrays, if coming from cosmic strings, will draw attention to interferometer searches for this source.

\subsection{Gravitational Waves From Phase Transitions}

Grand unification models can accommodate a rich scalar sector, which can result in a complicated cosmological history involving exotic phase transitions. Some GUT inspired possibilities are: a color breaking phase transition where color is broken and restored when leptoquarks acquire a vacuum expectation value in an intermediate transition, which can catalyse baryogenesis [317, 318], $B-L$ and $L$ violating phase transitions [319-322], hidden sector phase transitions [323-326], and a Pati-Salam transition [327]. If any such phase transition occurs through bubble nucleation, an observable relic gravitational wave spectrum can be seen today, for a review see Mazumdar and White [328], Caprini and Figueroa [329], and Weir [330]. Furthermore, GUT models often require the existence of extra singlets. For example, the $\mathrm{E}_{6} \mathrm{SSM}$ model studied in Athron et al. [241] had 3 generations of singlets and such singlets can, in principle, catalyse the electroweak phase transition to be strongly first order as well [331-333].

The gravitational wave spectrum generated from a cosmic phase transition has three contributions: a contribution from the collision of scalar shells, and potentially long-lasting contributions from sound waves and turbulence in the plasma. The total spectrum can thus be written as,

$$
\Omega(f) h^{2}=\Omega_{\mathrm{coll}}(f) h^{2}+\Omega_{\mathrm{sw}}(f) h^{2}+\Omega_{\mathrm{turb}}(f) h^{2} .
$$

Although much uncertainty remains about the precise form of these spectra, all three are controlled by four thermal parameters, which can be computed by first principles $[328,334]$ : the latent heat released during the transition (conventionally normalized by the radiation energy density), denoted $\alpha$, the nucleation rate (conventionally normalized to the Hubble parameter) $\beta / H$, the temperature at which the transition occurs $T_{*}$ and the velocity of the bubble wall $v_{w}$. The collision term is expected to be subdominant for transitions associated with a broken gauge group
[335]. The sound wave contribution to the power spectrum is [334] and [336]

$$
h^{2} \Omega_{\mathrm{sw}}=8.5 \times 10^{-6}\left(\frac{100}{g_{*}}\right)^{-1 / 3} \Gamma^{2} \bar{U}_{f}^{4}\left(\frac{\beta}{H}\right)^{-1} v_{w} S_{\mathrm{sw}}(f),
$$

where $\bar{U}_{f}^{2} \sim(3 / 4) \kappa_{f} \alpha_{T}$ is the rms fluid velocity, $\Gamma \sim 4 / 3$ is the adiabatic index, $\kappa_{f}$ is the efficiency of converting the latent heat into gravitational waves and $g_{*}$ the number of relativistic degrees of freedom. The frequency dependence is captured by the spectral state

$$
\begin{array}{r}
S_{\mathrm{sw}}=\left(\frac{f}{f_{\mathrm{sw}}}\right)^{3}\left(\frac{7}{4+3\left(\frac{f}{f_{\mathrm{sw}}}\right)^{2}}\right)^{7 / 2}, \\
f_{\mathrm{sw}}=8.9 \times 10^{-7} \mathrm{~Hz} \frac{1}{v_{w}}\left(\frac{\beta}{H}\right)\left(\frac{T_{*}}{\mathrm{Gev}}\right)\left(\frac{g_{*}}{100}\right)^{1 / 6} .
\end{array}
$$

The other notable, albeit sub-dominant, contribution is the contribution from magneto-hydrodynamic turbulence in the plasma. The power spectrum from this contribution is given by [337],

$$
\begin{aligned}
h^{2} \Omega_{\text {turb }}=3.354 \times 10^{-4} & \left(\frac{\beta}{H}\right)^{-1}\left(\frac{\kappa \epsilon \alpha}{(1+\alpha}\right)^{3 / 2} \\
& \left(\frac{100}{g^{*}}\right)^{1 / 3} v_{w} S_{\text {turb }}(f),
\end{aligned}
$$

where $\epsilon$ is the fraction of the energy that contributes to turbulence, typically taken to be in the range $(0.05,0.1)$ [334]. In this case the spectral form is a function of two time scales,

$$
\begin{array}{r}
S_{\text {turb }}=\frac{\left(f / f_{\text {turb }}\right)^{3}}{\left[1+\left(f / f_{\text {turb }}\right)\right]^{11 / 3}\left(1+\frac{8 \pi f}{h_{*}}\right)}, \\
f_{\text {turb }}=27 \mu \mathrm{Hz} \frac{1}{v_{w}}\left(\frac{T_{N}}{100 \mathrm{GeV}}\right) \frac{\beta}{H}\left(\frac{g^{*}}{100}\right)^{1 / 6},
\end{array}
$$

where $h^{*}$ is the Hubble rate at the transition temperature ${ }^{9}$.

For a single scalar field transition, without a tree-level barrier between the true and the false vacuum, $\beta / H$ tends to be $O\left(10^{3}\right)$ or greater [232]. The transition temperature is the same order of magnitude as the mass of the scalar. Therefore, transitions with scalar masses $O\left(10^{5}\right) \mathrm{GeV}$ can be probed by ground-based interferometers such as the Einstein Telescope [338], Kagra [339] and cosmic explorer [340], whereas space-based LISA will probe transitions at the electroweak scale [334]. The former can be more directly related to studies of GUTs-we show benchmark examples for a Pati-Salam phase transition are shown in Figure 7. The visibility of the spectrum tends to grow with the ratio $v / m$, the gauge coupling constant $g$, the rank of the (sub) group being broken and the number of other particles acquiring a mass during

\footnotetext{
${ }^{9}$ Note that the existence of two time scales in the spectral form means that the peak amplitude for the turbulence contribution cannot be found simply be setting the frequency to either $h_{*}$ or $f_{\text {turb }}$ in Equation 55.
} 


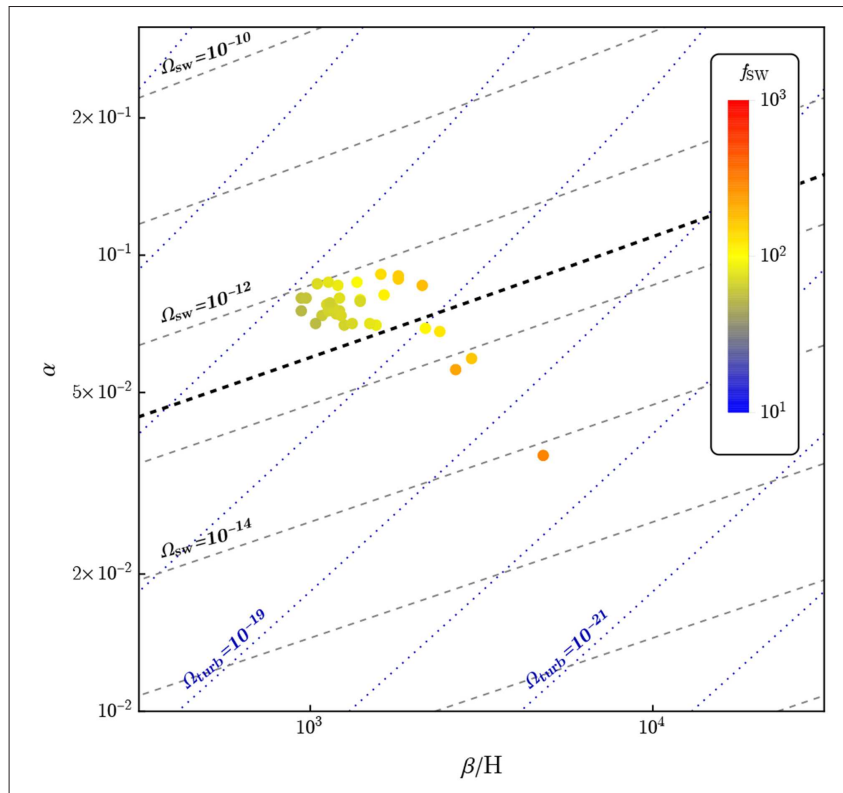

FIGURE 7 | Plane of thermal parameters with contours of constant sound wave (in black) and turbulence (in blue) peak amplitude. The thicker line shows the peak sensitivity of the Einstein telescope [338]. Points denote thermal parameters produced by a Pati-Salam phase transition with a Pati-Salam scale of $M_{P S}=10^{5} \mathrm{GeV}$. The peak frequency for the sound wave spectrum is indicated by the color scaling. Figure taken from Croon et al. [327].

the transition [323]. Furthermore, it was found in Croon et al. [323] that some non-trivial model discrimination is possible if one observes a primordial power spectra due to the increase in visibility as well as moderate correlations between thermal parameters, shown in Figure 8 for $S U(N) / S U(N-1)$ cosets.

If multiple scalar fields are involved in a transition the barrier between the true and false vacuum can persist at zero temperature due to triscalar or non renormalizable operators $[333,343]$. In such a case significantly more supercooling is possible and the transition temperature is no longer confined to be the same order of magnitude as the scalar mass. This implies that $\beta / H$ can be quite small and the latent heat can be large, increasing the visibility of the gravitational wave and reducing the peak frequency. A caveat to this is that recent work found that phase transitions that involve a large amount of supercooling may fail to complete due to the onset of inflation [344]. Regardless, the thermal parameter space in the case of multifield phase transitions is broader, which minimizes model discrimination somewhat, though not completely [323].

\subsection{Baryo-/Leptogenesis}

The existence of a baryon asymmetry in the Universe (BAU) is one of the central problems of modern cosmology [345, 346]. At the same time, the concordance between different measurements of the primordial baryon asymmetry is a triumph of modern cosmology with BBN and CMB limits giving [347, 348]

$$
\eta_{B}=\left\{\begin{array}{cc}
(6.2 \pm 0.4) \times 10^{-10} & \mathrm{BBN} \\
(6.14 \pm 0.03) \times 10^{-10} & \mathrm{CMB}
\end{array}\right.
$$

respectively. Any explanation for the baryon asymmetry must satisfy the three Sakharov conditions [349]

- Baryon number $B$ violation

- $\mathrm{C}$ and $\mathrm{CP}$ violation

- A departure from thermal equilibrium.

Early attempts at generating the BAU focused on $B$ violating decays (for a review see [350]). Such decays typically violate $B+L$ while conserving $B-L$ (for an exception see [351]). For example, $S U(5)$ GUTs are invariant under changes to a global phase conjugate to $B-L$ number, whereas $S O(10)$ has a local $U(1)_{B-L}$ symmetry. However, any primordial $B+L$ asymmetry is washed out by $B+L$ violating electroweak sphalerons. Therefore, only a primordial $B-L$ asymmetry will be preserved unless sphalerons are quenched.

Leptogenesis allows for a $B-L$ violating operator, $m \bar{v}_{R}^{c} v_{R}$, that is also responsible for a light neutrino mass via typeI seesaw mechanism (see section 3.2). CP violating decays of such sterile neutrinos ensure a net $B-L$ asymmetry which electroweak sphalerons convert to a baryon asymmetry. Electroweak baryogenesis by contrast uses the sphalerons themselves to generate a net $B+L$ asymmetry which cannot be washed out before the sphalerons are quenched [345, 346]. More specifically, if the electroweak phase transition is strongly first order, bubbles of electroweak broken phase populate a medium of symmetric phase with sphalerons quenched only inside such bubbles. Particles can have $\mathrm{CP}$ violating interactions with the bubble wall which biases the sphalerons to produce a net $B+L$ asymmetry. Some of this asymmetry is swept up in the expanding bubble wall where it is preserved.

GUTs are only relevant to electroweak baryogenesis if the GUT model motivates some light BSM states. Recent work on electroweak baryogenesis in the $E_{6}$-SSM utilized three generations of singlet superfields to motivate a CPV source involving singlino-Higgsino interactions with the bubble wall [331].

A feature of leptogenesis during GUTs is the possibility of new $\mathrm{CP}$ violating decay channels due to the presence of leptoquarks $[352,353]$. This allows a lower minimum mass for the lightest sterile neutrino than in the minimal scenario [236 ${ }^{10}$. Much of the recent focus on baryogenesis within GUTs involves leptogenesis with some intriguing concordance achieved in the case of $S U(5)$ [355] and SO(10) GUTs [271, 278, 356-360]. A generic feature of $S O(10)$ GUTs is normal ordering of neutrino masses and a negative Dirac phase [361], both of which are favored by current observational limits [360]. Furthermore, many GUTs, including $S O(10)$, predict a Dirac neutrino mass matrix that is not too different from the up quark mass matrix and $S O(10)$ leptogenesis also achieves agreement in the currently observed atmospheric mixing angle in the first octant. Realistic models with two right-handed neutrinos can emerge in $\Delta(27) \times S O(10)$ models and $A 4 \times S U(5)$ supersymmetric models [362, 363]. The third right-handed neutrino can either decouple because it is very heavy or because its Yukawa coupling is very small. The ${ }^{10}$ This limit of course is for the non resonant regime. In case of resonant
leptogenesis the masses of the sterile neutrinos can be very low [354]. 

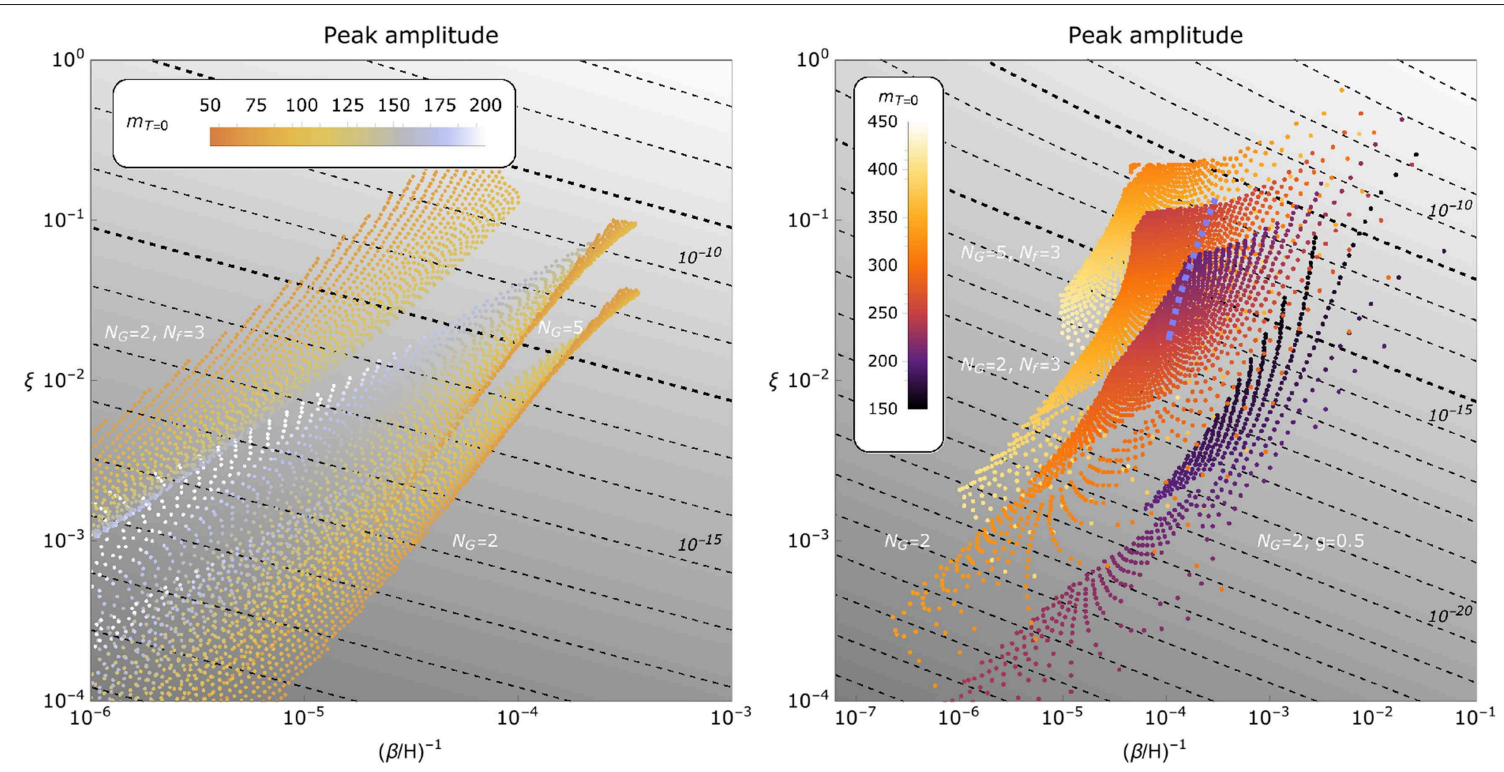

FIGURE 8 | Thermal parameters from a renormalizable potential for a scalar field in the fundamental representation of $S U\left(N_{G}\right)$ which gets broken to $S U\left(N_{G}-1\right)$ (left) Right panel is the same aside from the inclusion of a non-renormalizable sextet term in the potential and the gauge coupling constant is fixed to unity. In the above $N_{G}$ denotes the order of the group, $N_{f}$ is the number of fermions in the fundamental representation that are strongly coupled to the scalar field, in direct analogy with the SM save that the Yukawa couplings are set to unity. Note that $\xi$ is the ratio of latent heat to radiation energy density more commonly denoted $\alpha$. Contours of constant peak amplitude for the sound wave spectrum are shown with the darker line corresponding to LISA sensitivity range for a power law spectrum that has been integrated over frequency [341]. Note in the above $v_{w}=0.5$ for the left plot and $v_{w}=1$ for the right plot using the efficiency terms in Espinosa et al. [342]. Figure taken from Croon et al. [323].

latter case implies a stable particle that can play the role of dark matter [364].

\section{DIRECT COLLIDER SEARCHES FOR GUTS}

\subsection{Searches for Supersymmetry}

As we have seen in section 3.1 supersymmetry plays a rather important role on many unified theories and motivates the unification of gauge couplings at large scales. However, both its solution to the hierarchy problem and gauge coupling unification often rely on a light sparticle spectrum, around or below the $\mathrm{TeV}$ scale. Thus, searches for supersymmetric particles has been part of the research programme in collider physics for the last few decades, from searches at LEP and the Tevatron, to the recent results of the $\mathrm{LHC}$, and it is still part of the proposed physics programme for future colliders, e.g., CLIC, ILC, or VLHC [365-368].

In R-parity conserving SUSY the lightest supersymmetric particle (LSP) is stable. This has strong consequences for SUSY searches, for the LSP will escape the collider in the form of missing transverse energy $(\mathrm{MET})^{11}$ [369]. In addition, R-parity requires that sparticles are pair-produced in colliders, hence the different searches for supersymmetry are classified according

\footnotetext{
${ }^{11}$ In cases where a charged next-to-lightest SUSY particle (NSLP) is stable at detector timescales, no clear MET signal is produced, since the NLSP will decay to the LSP outside the detector.
}

to the particle that is produced in pairs. The production cross sections of the different sparticle species are rather different and often determine the exclusion or detection power of a particular channel. For instance, the strongest exclusion limits at the LHC across the sparticle spectrum are on first and second generation squarks and gluinos which, as can be seen in Figure 9, have the largest production cross sections [370, 371].

Squarks and gluinos are produced in pairs at the LHC in the combinations $\tilde{g} \tilde{g}, \tilde{q} \tilde{q}$ and $\tilde{q} \tilde{g}$ and their main decay channels are $\tilde{q} \rightarrow q \tilde{\chi}_{1}^{0}$ and $\tilde{g} \rightarrow q \bar{q} \tilde{\chi}_{1}^{0}$, with the neutralino LSP escaping the collider. Hence the typical signature for these processes has multiple jets and large missing energy. The decay topologies for these signatures are depicted in Figure 10. Other decay modes for squark and gluinos involve the production of charginos or heavier neutralinos, $\tilde{q} \rightarrow q \tilde{\chi}_{2}^{0}, \tilde{q} \rightarrow q^{\prime} \tilde{\chi}_{1}^{ \pm}, \tilde{g} \rightarrow q \bar{q} \tilde{\chi}_{2}^{0}$ and $\tilde{g} \rightarrow q \bar{q} \tilde{\chi}_{1}^{ \pm}$, which then decay to $W$ and $Z$ bosons and $\tilde{\chi}_{1}^{0}$. The final state signatures depend on the decay modes of the gauge bosons, and can have (0-4) leptons, jets and MET. ATLAS and CMS have reported results from the last run of the LHC at $13 \mathrm{TeV}$ and $36 \mathrm{fb}^{-1}$ for searches with jets and MET final states [372-374], with one lepton, jets and MET [375-377], same and opposite-sign dilepton pairs, jets and MET [378, 379], two or three leptons, jets and MET [380, 381], 3rd generation squarks (with and without Higgs reconstruction) and MET [382-384] and hadronic $\tau$ decays, jets and MET [385], among others. These searches set a lower limit for a range of simplified models on the mass of the gluino of $m_{\tilde{g}} \gtrsim 2.1 \mathrm{TeV}$ and the mass of the first and second generation squarks of $m_{\tilde{q}} \gtrsim 1.5 \mathrm{TeV}$. 
The next strongest production cross section is that of stop and sbottom pairs. The main decay channel for stops and sbottoms is $\tilde{t} \rightarrow t \tilde{\chi}_{1}^{0}$ and $\tilde{b} \rightarrow b \tilde{\chi}_{1}^{0}$, respectively. This topology is similar to the decay of first and second generation squarks, with the added complexity that neither $t$ or $b$ produce a clean jet, but rather have many decay channels that can result in numerous jets, leptons and, of course, MET. Secondary decay channels for $\tilde{t}$ and $\tilde{b}$ involve decays to a chargino, $\tilde{t} \rightarrow b \tilde{\chi}_{1}^{ \pm}$and $\tilde{b} \rightarrow t \tilde{\chi}_{1}^{ \pm}$, with subsequent decays involving $W$ bosons, or decays into a heavy neutralino, $\tilde{t} \rightarrow t \tilde{\chi}_{2}^{0}$ and $\tilde{b} \rightarrow b \tilde{\chi}_{2}^{0}$, which in turn decays into a $Z$ or a Higgs boson and $\tilde{\chi}_{1}^{0}$. The latest searches of the LHC experiments for pair-produced stops and sbottoms target final states with jets and MET [374, 386, 387], b-jets and $\operatorname{MET}[388,389]$, one lepton, jets and $\operatorname{MET}[390,391]$, two and three leptons, jets and MET [379-381, 392, 393] and final states with a $h$ or a $Z$ boson and MET [394], among others. These searches exclude masses of stops and sbottoms up to $m_{\tilde{t}} \sim 1 \mathrm{TeV}$ and $m_{\tilde{b}} \sim 900 \mathrm{GeV}$ for some simplified models.

In the cases where the colored sector of a supersymmetric model has large masses, the direct production of chargino, neutralino and slepton pairs dominate. A pair of directly produced sleptons decay typically like $\tilde{l} \rightarrow l \tilde{\chi}_{1}^{0}$. Neutralinos and charginos are produced in pairs in a number of different

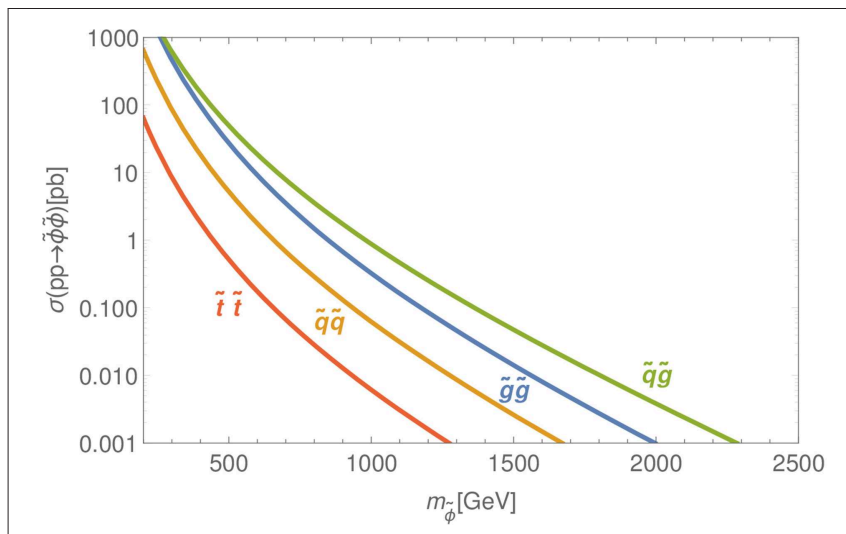

FIGURE 9 | Pair production cross sections as a function of the mass of sparticle $\tilde{\phi}$ at center of mass energy $\sqrt{s}=13 \mathrm{TeV}$. combinations, the most commonly studied of which are $\tilde{\chi}_{2}^{0} \tilde{\chi}_{1}^{ \pm}$ and $\tilde{\chi}_{1}^{ \pm} \tilde{\chi}_{1}^{ \pm}$. The decays of heavy neutralinos and charginos produce $W, Z$ or Higgs bosons and the lightest neutralino. Further decay of $W$ and $Z$ sets the final states targeted by ATLAS and CMS searches, such as the final state with two leptons and MET [395-397], many leptons and MET [397-400], leptons, jets and MET [393], taus and MET [401, 402], and b-jets plus MET [403], among others. Due to their low production cross sections, the exclusion limits on slepton masses from direct production are quite weak and they only reach up to around $m_{\tilde{l}} \sim$ $500 \mathrm{GeV}$. Stronger limits on slepton masses can be inferred from neutralino/chargino production with sleptons in the cascade, reaching up to $\sim 850 \mathrm{GeV}$. The limits on electroweakinos (neutralinos and charginos) are very sensitive to the parameter choices for the simplified model analyses performed by the experiments, hence the exclusion limits on $\tilde{\chi}_{2}^{0}$ and $\tilde{\chi}_{1}^{ \pm}$vary from search to search and from signal region to signal region, roughly in the range $m_{\tilde{\chi}_{2}^{0}}, m_{\tilde{\chi}_{1}^{ \pm}} \in(500 \mathrm{GeV}, 1.1 \mathrm{TeV})$. Similarly the limits on the lightest neutralino varies in the range $m_{\tilde{\chi}_{1}^{0}} \in(200,700)$ $\mathrm{GeV}$. In addition, there is a hint of an excess in one of the two and three lepton final state analyses by the ATLAS collaboration in the low $m_{\tilde{\chi}_{1}^{0}}$ region, with a reported significance of 2 and $3 \sigma$ in the $2 l$ and $3 l$ channels, respectively [399].

In addition to sparticle searches, SUSY can also be probed through searches for heavy and charged Higgs bosons. The MSSM predicts the existence of two CP-even scalars, $h$ and $H$, one CP-odd pseudoscalar, $A$, and a charged scalar $H^{ \pm}$. The lightest CP-even scalar, $h$, is said to be "SM-like," as its mass and couplings are aligned with the Higgs boson discovered at the LHC $[4,5]$, the so called alignment limit. Neutral heavy Higgses can be produced at the LHC in the same manner as the SM Higgs, that is by gluon fusion, vector boson fusion (VBF) and associated production, with a $t$ and/or $b$ quark. Thus, the same mechanisms that lead to the discovery of the SM Higgs are employed to set exclusion limits on heavier neutral (pseudo)scalars, including signatures where $H$ is produced in resonance and decays into two light Higgs bosons $p p \rightarrow H \rightarrow h h$. The final states targeted by these exotic Higgs searches consist of 2-4 leptons, jets and MET from on- and offshell $W$ and $Z$ bosons [404, 405], two leptons and MET [406, 407], final states with four b-jets [408, 409] or two b-jets and $W W[410], \gamma \gamma[411,412], \tau \tau[413,414], \mu \mu$ [415], or $t \bar{t}$ [416]
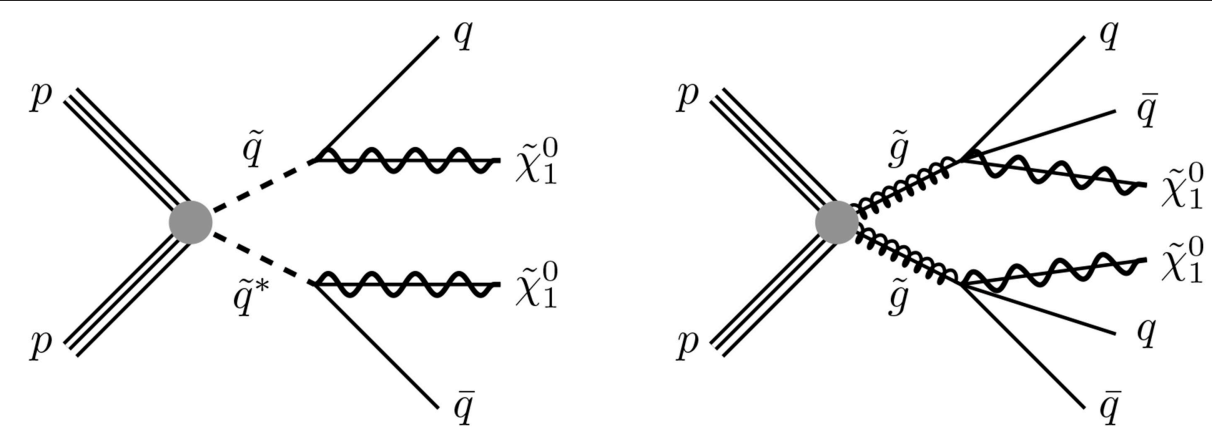

FIGURE 10 | Most simple topologies for the production of squarks and gluinos at the LHC with decays to jets + MET. 
decays, ditau final states $[417,418]$ and diphotons, with and without associated $W$ bosons [419-421]. Charged Higgs bosons, $H^{ \pm}$can typically be produced with associated resonant and nonresonant top-quark production. Their main decay channels are $H^{ \pm} \rightarrow W^{ \pm} Z[422,423], H^{ \pm} \rightarrow t(c) b[416,424]$ and $H^{ \pm} \rightarrow$ $\tau^{ \pm} v[416,425]$. Since no excess has been found for either heavy $H$ or $H^{ \pm}$, the experiments set upper limits that strongly depend on the production cross section and, in turn, on $\tan \beta$. For $H$ the limits range from $m_{H}<400 \mathrm{GeV}$ for $\tan \beta=2$ and production cross sections limits of $0.1 \mathrm{pb}$ for larger masses. For $H^{ \pm}$with $m_{H^{ \pm}}<160 \mathrm{GeV}$ excluded for all values of $\tan \beta$ and $m_{H^{ \pm}}<1.1$ $\mathrm{TeV}$ excluded for $\tan \beta=60$.

Many unified theories automatically preserve $R$-parity, such as left-right symmetric or Pati-Salam models, as well as supergroups of them, $S O(10)$ or $E_{6}$. This is because they contain a gauged $U(1)_{B-L}$ subgroup which effectively makes the LSP stable [76]. Other models, such as $S U(5)$, may have $R$-parity violating (RPV) interactions, though in general they will be suppresed since they can lead to rapid proton decay. Since the LSP is no longer stable, collider signatures of RPV typically contain multiple leptons [400], multiple jets [426-429] or both [430, 431] in the final state. These searches often impose strong upper limits on sparticle masses that range from $150 \mathrm{GeV}$ to a few $\mathrm{TeV}$ for $\tilde{t}$, depending on the channel, and from 1 to 2 $\mathrm{TeV}$, for $\tilde{g}$.

If the LSP is metastable or the lightest chargino and neutralino are almost degenerate, they can live long enough to leave a displaced vertex or a disappearing track on the detector. Detailed searches have been performed by ATLAS [432-436] and CMS [437-439] to search for these long-lived particles, and they have reached exclusion limits comparable to those of the detailed searches above.

Most of the searches described above assumed a neutralino LSP, which is typically the case in gravity mediated SUSY breaking. In gauge mediated SUSY breaking (GSMB) and general gauge mediation (GGM) the LSP is actually a nearly massless gravitino. In these cases new decay channels are open with photons [440-443], Z's [400, 403], Higgses $[400,403,444]$, and $\tau s[385,445]$ in the final state (see Figure 11).

Although the LHC results are the most recent and, for the most part, they supersede the results of previous collider experiments, such as those at the Tevatron, some experimental limits from LEP still remain relevant today. In particular for models with a significant production of neutralino/chargino or slepton pairs, the limits from ALEPH [446, 447], OPAL [448, 449], L3 [450, 451], and DELPHI [452] on sleptons and electroweakino masses are rather pertinent, as they are largely model independent.

Many of the SUSY searches above are performed using simplified models, e.g., ATLAS jets and MET search assumes a 50-50 split between the decay modes of gluinos [372]. Therefore, the mass and cross section limits obtained are weakened in more complicated models. In order to assess the relevance of many of these exclusion limits on several popular SUSY models, a full global fit of the parameter space of the model is required. Several of these fits have been performed for the CMSSM, NUMH1 and NUMH2 [453-459], phenomenological MSSM models [460462], SUSY GUT models [463] and electroweak-sector MSSM models [116], by the Zfitter [464] ${ }^{12}$, SuperBayes $[465,466]^{13}$, Fittino $[467]^{14}$, MasterCode $[468]^{15}$, and GAMBIT $[469-476]^{16}$ collaborations.

\subsection{Collider Searches for Leptoquarks}

Leptoquarks (LQs) are associated either with the vector (spin 1) particles that correspond to the gauge bosons of the unified gauge group or they can be scalars (spin 0) and belong to a Higgs sector of a unified theory. Vector LQ mass is typically of the order of the unification scale and can only be accessible directly at colliders if the unification scale is low enough (e.g., Pati-Salam models). Scalar representations can also contain light fields, most notably the SM Higgs, but come at the cost of severe fine tuning, as discussed in the section 2 on the example of doublet-triplet splitting problem in 5 -dim. representation of $S U(5)$. In non-supersymmetric unified models the presence of light colored scalars tends to aid unification (see e.g., [477]). Another important difference is that the scalar LQ interactions can be analyzed without specifying the concrete GUT completion in the ultraviolet. On the other hand, vector (gauge) LQs are sensitive to the mass generation mechanism that is specified in the ultraviolet. Therefore, effective vector LQ models are not renormalizable [478]. Furthermore, even the couplings of vector LQs to the SM gauge sector are not completely fixed by the gauge quantum numbers [479, 480].

Altogether there are six scalar and six vector leptoquarks, listed in Table 1, that couple to the SM matter at the renormalizable level [480-482]. The fermionic number $F \equiv$ $3 B+L$ of leptoquarks that do not couple to diquarks $(\phi q q)$ and are potentially $B$ and $L$ conserving, must be $F=0$, whereas LQs with $|F|=2$ in general destabilize the proton via $B-L$ conserving decays.

The most important phenomenological characteristic of light LQs (of mass of the order few $\mathrm{TeV}$ ) is their color triplet nature allowing them to be produced in pairs via strong interactions in a largely model independent manner. In this section we will focus on the on-shell production of LQs at $p p$ colliders, since the current mass constraints are dominated by LHC searches. For specific signatures of LQ production in colliders with alternative initial states (see [480]). Pair production of leptoquarks is model independent for the $g g \rightarrow$ LQLQ partonic process, while the parton level process $q \bar{q} \rightarrow$ LQLQ is affected also by the $t$-channel lepton exchange diagram (bottom right diagram in Figure 12) that introduces some model dependence when the leptoquark flavor couplings are non-negligible. The partonic cross sections for pair production at leading order

\footnotetext{
12 zfitter.desy.de/

${ }^{13}$ www.ft.uam.es/personal/rruiz/superbayes

14 flcwiki.desy.de/Fittino

${ }^{15}$ cern.ch/mastercode/

${ }^{16}$ gambit.hepforge.org
} 

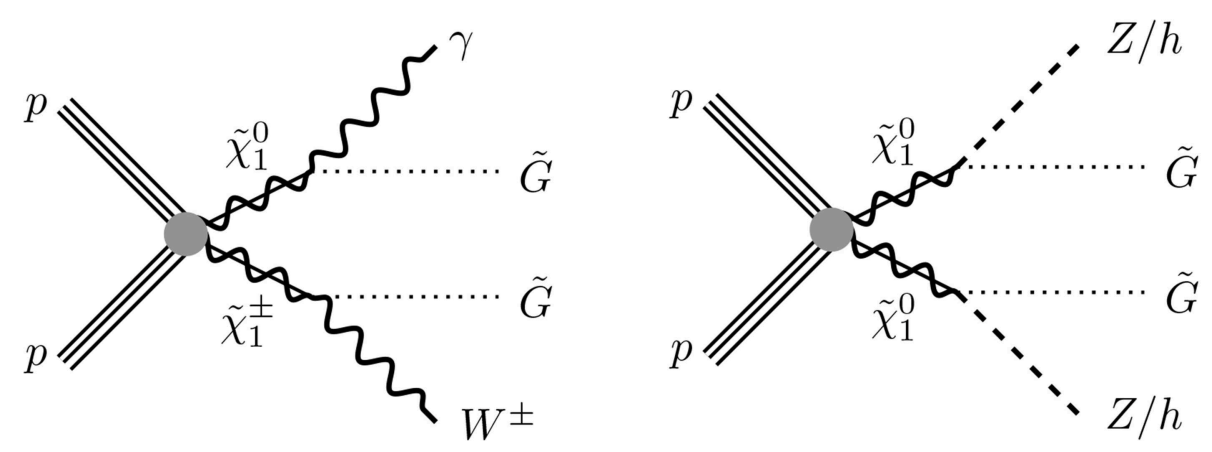

FIGURE 11 | Topologies for SUSY searches with gravitino LSP and $\gamma$ and $h / Z$ final states.

TABLE 1 | List of scalar and vector LQs.

\begin{tabular}{lccc}
\hline LQ symbol & {$[\mathbf{S U}(\mathbf{3}), \boldsymbol{S} U(\mathbf{2}), \boldsymbol{U}(\mathbf{1})]$} & Spin & $\boldsymbol{F}$ \\
\hline$S_{3}$ & $(\overline{\mathbf{3}}, \mathbf{3}, 1 / 3)$ & 0 & -2 \\
$\tilde{S}_{1}$ & $(\overline{\mathbf{3}}, \mathbf{1}, 4 / 3)$ & 0 & -2 \\
$S_{1}$ & $(\overline{\mathbf{3}}, \mathbf{1}, 1 / 3)$ & 0 & -2 \\
$\bar{S}_{1}$ & $(\overline{\mathbf{3}}, \mathbf{1},-2 / 3)$ & 0 & -2 \\
$R_{2}$ & $(\mathbf{3}, \mathbf{2}, 7 / 6)$ & 0 & 0 \\
$\tilde{R}_{2}$ & $(\mathbf{3}, \mathbf{2}, 1 / 6)$ & 0 & 0 \\
$U_{3}$ & $(\mathbf{3}, \mathbf{3}, 2 / 3)$ & 1 & 0 \\
$\tilde{U}_{1}$ & $(\mathbf{3}, \mathbf{1}, 5 / 3)$ & 1 & 0 \\
$U_{1}$ & $(\mathbf{3}, \mathbf{1}, 2 / 3)$ & 1 & 0 \\
$\bar{U}_{1}$ & $(\mathbf{3}, \mathbf{1},-1 / 3)$ & 1 & 0 \\
$V_{2}$ & $(\overline{\mathbf{3}}, \mathbf{2}, 5 / 6)$ & 1 & -2 \\
$\tilde{V}_{2}$ & $(\overline{\mathbf{3}}, \mathbf{2},-1 / 6)$ & 1 & -2 \\
\hline
\end{tabular}

are [483-488]:

$$
\begin{aligned}
& \hat{\sigma}(g g \rightarrow \phi \bar{\phi}) \\
& =\frac{\alpha_{3}^{2} \pi}{96 \hat{s}}\left[\beta\left(41-31 \beta^{2}\right)+\left(18 \beta^{2}-\beta^{4}-17\right) \log \frac{1+\beta}{1-\beta}\right], \\
& \hat{\sigma}(q \bar{q} \rightarrow \phi \bar{\phi})=\frac{2 \alpha_{3}^{2} \pi}{27 \hat{s}} \beta^{3},
\end{aligned}
$$

where $\hat{s}$ is the partonic center-of-mass energy squared, $\alpha_{3}$ is the strong coupling constant, and $\beta=\sqrt{1-4 m_{\phi}^{2} / \hat{s}}$. A model independent study of weak doublet scalar LQs at the LHC and the interplay with low energy flavor processes was performed in [489], where it was also shown that searches for single LQ production could be more sensitive in the regime of large Yukawas and/or LQ masses. An analysis of pair and single production, along with the corresponding UFO model files LQ_NLO, both for scalar and a vector leptoquark has been presented in Doršner and Greljo [490].

On the other hand, single leptoquark production at $p p$ colliders is always model dependent (Figure 13). Single leptoquark searches are more effective at larger LQ masses [489].

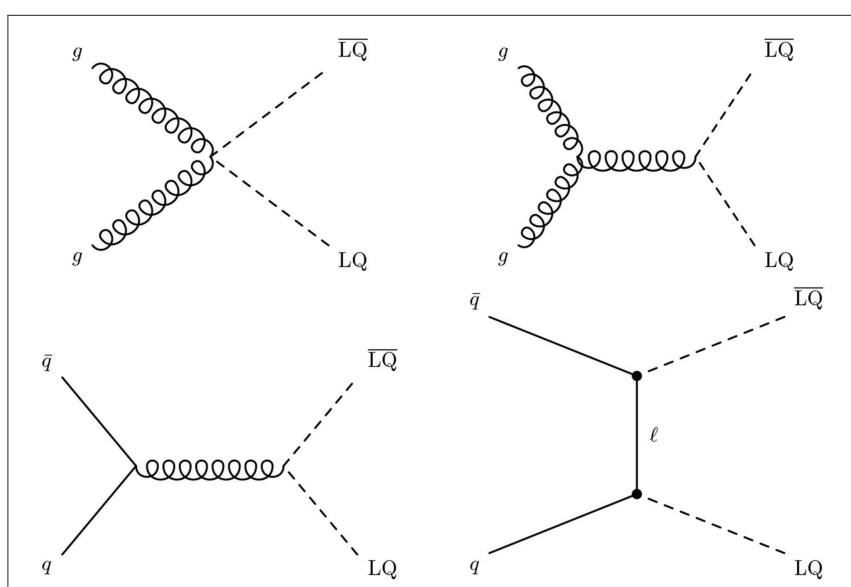

FIGURE 12 | Representative diagrams for leptoquark pair production at $p p$ colliders. Dots denote the LQ- $q-\ell$ coupling.

On the decay side of the process, experimental searches for pair and single LQ production are targeting a resonance in the $j \ell$ channel. The decay width of a scalar leptoquark into a lepton-quark final state is given by [490] and [491]:

$$
\Gamma(\phi \rightarrow q \ell)=\frac{\left|y_{q}\right|^{2} m_{\phi}^{2}}{16 \pi}\left[1+\frac{\alpha_{s}}{\pi}\left(\frac{9}{2}-\frac{4 \pi^{2}}{9}\right)\right] .
$$

Current bounds from dedicated leptoquark pair production have been commonly extracted in the framework that assumed LQ coupling only to a single generation of SM fermions, whereas realistic LQ scenarios could posses richer flavor structure [480, 492]. The experimental upper bounds are given for the product of cross section and the LQ branching fraction probability $\beta^{2}$, where $\beta$ is the probability for LQs to decay to a final state with charged leptons. There have been numerous analyses performed at the LHC for leptoquarks being either of 1st [493], 2nd [494], or 3rd [495-499] generation. More recent studies, motivated by the observed lepton universality violation in $B$-meson decays, allow also for cross-generational couplings, e.g., [500]. Finally, also single LQ production 


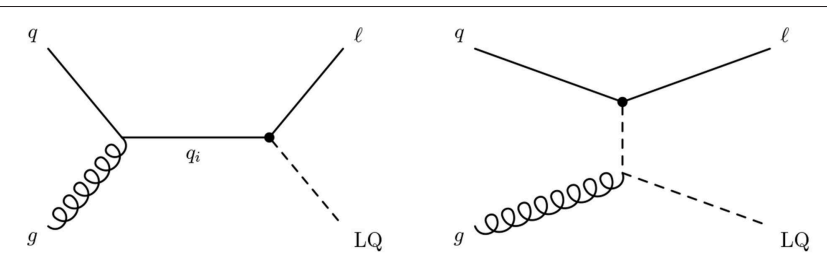

FIGURE 13 | Representative diagrams for single leptoquark production at $p p$ colliders. Dots denote the LQ- $q-\ell$ coupling.

channels are being studied [501]. To conclude, the current lower bounds on leptoquark masses from direct searches at the LHC range from several $100 \mathrm{GeV}$ to above the $\mathrm{TeV}$ scale, where the exact bound depends on the size of the flavor couplings.

\subsection{Other Exotic Searches}

Many unified theories include heavy sterile neutrinos that contribute to the mass of the light neutrinos via type-I seesaw mechanism (see section 3.2). These are often associated with the symmetry breaking of a left-right sector of the theory, and thus they are expected to be heavier than the EW scale. Direct searches for heavy neutrinos at colliders often target a decay channel where final state has two same-sign leptons, via $s$ or $t$-channel production of a gauge boson that can be left or right handed [502]. Figure 14 shows the Feynman diagram for the golden channel for heavy neutrino searches, $p p \rightarrow$ $W \rightarrow N l \rightarrow W l l \rightarrow l l j j$. The ATLAS and CMS experiments at the LHC have performed searches for heavy neutrinos in LR models with masses $M_{N} \approx(20,1,600) \mathrm{GeV}$ and have imposed strong limits on the couplings between active and sterile neutrinos [503-505].

Other searches for sterile neutrinos are performed in beam dump experiments [506], where the neutrinos are produced in semileptonic decays of mesons, with masses below $2 \mathrm{GeV}$ [507-510]. For intermediate masses below the $Z$ resonance, the strongest limits come from the decay $Z$ bosons by the LEP experiments DELPHI and L3 [511, 512].

In addition to singlet fermions and colored leptoquarks, the LHC looks for heavy colorless vector bosons as part of their exotic searches programme. Charged $W^{\prime}$ and neutral $Z^{\prime}$ vector bosons are predicted in a number of GUT frameworks and they can often live at low scales, which positions them within the reach of colliders. Clear examples of this are the left-right symmetric models described in section 4.1, that predict light $W_{R}$ and $Z_{R}$ bosons, or the light $Z_{N}$ appearing in $\mathrm{E}_{6} \mathrm{SSM}$ models .

These states are produced at $p p$ colliders through DrellYan processes $p p \rightarrow W^{\prime} / Z^{\prime}$ and subsequently decay into leptons or jets. One of the most targeted processes for $W^{\prime}$ involve the decay into heavy neutrinos, as in Figure 14, with two same or opposite sign leptons (depending on the Majorana or Dirac nature of the heavy neutrinos) and jets [503, 504]. These searches often use a simplified model where $g_{R}=$ $g_{L}$ and $M_{N}=M_{W}^{\prime} / 2$ resulting in high exclusion limits with $M_{W^{\prime}} \gtrsim 4.5 \mathrm{TeV}$, but it has been shown that these

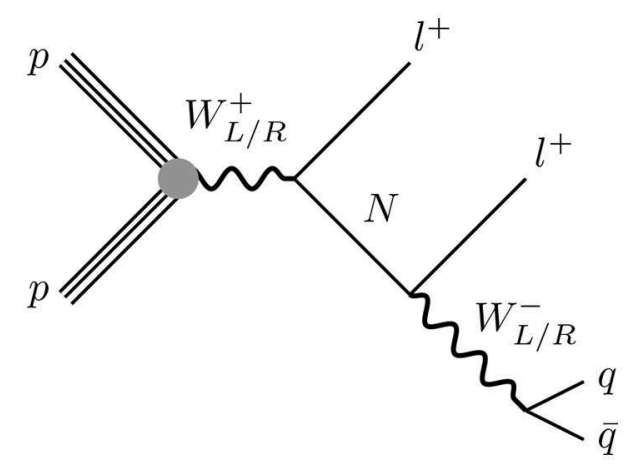

FIGURE 14 | Diagram for direct searches of neutrinos via $W_{L / R}$ production.

limits weaken somewhat in more general models [173-175]. CMS also reported a search for $W^{\prime}$ where the vector boson decays to $\tau \bar{\nu}_{\tau}$, the $\tau$ decaying hadronically [513], with slightly weaker limits.

Narrow resonance searches for $Z^{\prime}$ have been performed by ATLAS and CMS, targeting final states with two opposite-sign leptons. These searches have yielded model-dependent exclusion limits on $M_{Z^{\prime}}$. For $E_{6}$-inspired $Z^{\prime}$, the limits vary around $M_{Z^{\prime}} \gtrsim$ $(3,3.5) \mathrm{TeV}$, whereas for LR models they are moderately stronger $M_{Z^{\prime}} \gtrsim 4 \mathrm{TeV}[514,515]$.

Lastly, GUTs predict a plethora of different scalar states that can be observed at the LHC if they are light enough, e.g., $\Delta_{L, R}$ in LR symmetric models. Searches for neutral and singly charged scalar bosons are identical to the searches for supersymmetric Higgs bosons in section 6.1, so we will not repeat them here. Doubly-charged scalars, such as the $\delta_{L}^{ \pm \pm} \in$ $\Delta_{L}$ in LR models, have been studied by ATLAS and CMS in multilepton final states [516, 517], diboson final states [518] and in long-lived particle studies [519] with model-dependent limits below $1 \mathrm{TeV}$.

\section{PRECISION TESTS OF UNIFICATION}

\subsection{Proton Decay}

Unified theories may contain gauge or scalar bosons that mediate transitions between leptons and quarks. These transitions violate baryon $B$ and lepton $L$ number separately and hence can cause the rapid decay of nucleons [520-529].

In the language of Effective Field Theory, nucleon decay transitions are generated by higher dimensional operators suppressed by the mass of the heavy mediator. The most relevant contribution to nucleon decay comes from dimension6 operators of the form $q q q l$, mediated either by a gauge or scalar boson. In SUSY GUTs, however, dimension 4 and 5 operators can appear, involving $R$-parity violating interactions and mixing among sfermions, respectively. Assuming the conservation of $R$ parity and minimal flavor violation $(\mathrm{MFV})$ in the sfermion sector, however, dimension 4 and 5 contributions can be made negligible [530-532]. Therefore, dimension 6 operators dominate the contributions 
to nucleon decay. These are, in general, model dependent, so calculating the decay width requires knowledge of the flavor structure at the GUT scale and varies among the different decay channels [533, 534]. However, with some simplifying assumptions one can approximate the decay width of the proton as [535]

$$
\Gamma_{p} \approx \alpha_{G U T}^{2} \frac{m_{p}^{5}}{M_{G U T}^{4}}
$$

There are several decay channels for the proton and neutron, each with a different experimental bound. The most stringent of them, known as the gold channel for proton decay is $p \rightarrow e^{+} \pi^{0}$, whose parton-level diagrams can be seen in Figure 15, and with a lower limit on the half-life, set by the Super-Kamiokande, of $\tau>1.6 \times 10^{34}$ years [43]. Other processes with slighly lower bounds are $\tau(p \rightarrow$ $\left.\mu^{+} \pi^{0}\right)>7.7 \times 10^{33}$ years [43], $\tau\left(p \rightarrow v K^{+}\right)>5.9 \times$ $10^{33}$ years [536] and $\tau\left(n n \rightarrow e^{ \pm} \mu^{\mp}\right)>4.4 \times 10^{33}$ years [537].

The next generation of experiments for nucleon decay has already been proposed. Hyper Kamiokande will take the place of Super-K and has a projected sensitivity in the golden channel $p \rightarrow e^{+} \pi^{0}$ of $\sim 10^{35}$ years [538]. The Deep Underground Neutrino Experiment (DUNE) [539] expects to improve the limit on $p \rightarrow v K^{+}$to $\sim 3 \times 10^{34}$ years. These increased limits will probe unified teories at the highest scales and could be the smoking gun for them. In case of a positive signal from either of these experiments, precision calculations of proton decay processes with controlled uncertainties will become invaluable $[84,540]$.

\subsection{Flavor Phenomenology of Light Leptoquarks}

Several flavor couplings of scalar leptoquarks to leptons and quarks, which are in general connected to the GUT contractions of scalar and fermionic representations, allow their virtual effects to be tested in low-energy flavor observables. Such are the decays of hadrons or leptons, precision observables at LEP, and static properties of particles such as dipole moments. On the high- $p_{T}$ front, the LHC is also becoming competitive as a flavor probe for virtual effects of particles that are too massive to be produced on-shell.

The correlations between lepton-quark-LQ couplings are determined at low scales by the weak isospin and hypercharge. As an example, consider the weak doublet leptoquark $R_{2}(3,2,7 / 6)$

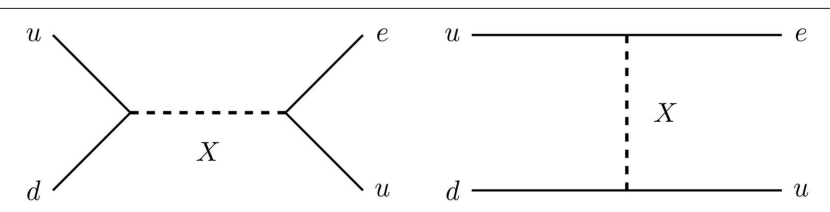

FIGURE 15 | Parton level $s$ and $t$-channel diagrams for the proton decay channel $\mathrm{p} \rightarrow \mathrm{e}^{+} \pi^{0}$ for a gauge or scalar boson mediator $X$. (see Table 1), which can couple to two-types of leptonquark bilinears:

$$
\begin{aligned}
\mathcal{L}= & Y_{R}^{i j} \bar{Q}_{i}^{\prime} \ell_{R j}^{\prime} R_{2}+Y_{L}^{i j} \bar{u}_{R i}^{\prime} \widetilde{R}_{2}^{\dagger} L_{j}^{\prime} \\
= & \left(V Y_{R}\right)^{i j} \bar{u}_{L i} \ell_{R j} R_{2}^{\frac{5}{3}}+Y_{R}^{i j} \bar{d}_{L i} \ell_{R j} R_{2}^{\frac{2}{3}} \\
& +Y_{L}^{i j} \bar{u}_{R i} v_{L j} R_{2}^{\frac{2}{3}}-Y_{L}^{i j} \bar{u}_{R i} \ell_{L j} R_{2}^{\frac{5}{3}} .
\end{aligned}
$$

Here $i, j$ are the flavor indices, primed fields are written in the interaction basis, unprimed fields are in the mass basis, except for the neutrinos which are aligned with charged leptons. There are three important features in the above Lagrangian. First, since $R_{2}$ is a weak doublet it must couple to lefthanded quark doublets, which implies that CKM matrix $V$ relates the couplings of up-type and down-type quarks. Second, $R_{2}$ couples to both chiralities of quarks and leptons, which leads to scalar and/or tensor effective interactions and could lead to enhanced effects in meson mixing amplitudes, dipole moments, and radiative decays [480]. Third, as $F=0$ for $R_{2}$ we cannot construct interaction term with diquark coupling, implying that proton cannot decay via $\Delta(B-L)=0$ process. Generalizing to other LQ states, weak triplets only talk to the left-handed fermions $(2 \otimes 2)$, leading to strictly chiral interaction, whereas singlet LQs can talk to $2 \otimes 2$ and $1 \otimes 1$ fermion bilinears.

Among the flavor constraints, leptoquarks naturally (at treelevel) contribute to semileptonic effective operators at low scales, therefore the most relevant observables are (semi-)leptonic decays of mesons, baryons, or $\tau$ leptons. The most notable charged-current and flavor changing neutral current (FCNC) constraints, and the general framework to address them in leptoquark models, have been spelled out in Leurer [541], Davidson et al. [482], and Doršner et al. [480]. The most constraining are the FCNC observables, where the tree-level LQ contribution can easily stand out of the SM signal, which is 1-loop suppressed in the case of quark FCNC and absent in the case of lepton FCNC. Effective dimension- 6 interactions for 4 lepton or 4 quark operators, which drive the $\ell \rightarrow$ $\ell^{\prime} \ell^{\prime} \ell^{\prime \prime}$ (see section 7.4) and meson mixing processes, occur at one-loop [480]. Therefore, meson mixing is in general not among the strictest constraints on LQs (for $B_{s}$ mixing see e.g., [542]).

\subsection{Lepton Flavor Universality}

Lepton flavor universality (LFU) ratios, defined as ratios between rates for processes that differ only in lepton flavor, are very well suited to test the validity of the SM. The main advantage is that in the Standard Model LFU is respected by all gauge interactions, the only breaking comes from mass splitting among leptons, which leads to efficient cancellation of hadronic and parametric uncertainties in LFU ratios. Recently, two LFU ratios in $B$-meson decays have been observed

$$
R_{D^{(*)}}=\frac{\mathcal{B}\left(B \rightarrow D^{(*)} \tau \bar{\nu}_{\tau}\right)}{\mathcal{B}\left(B \rightarrow D^{(*)} l \bar{\nu}_{l}\right)}, \quad R_{K^{(*)}}=\frac{\mathcal{B}\left(B \rightarrow K^{(*)} \mu \mu\right)}{\mathcal{B}\left(B \rightarrow K^{(*)} e e\right)},
$$


where $l=e, \mu$. Several experiments found that the ratios $R_{D^{(*)}}$ are larger than $R_{D^{(*)}}^{\mathrm{SM}}$. The measurements of $R_{D}[13,543,544]$ differ by $\sim 2 \sigma$ with respect to the SM prediction [545] and by $\sim 3 \sigma$ in the case of $R_{D^{*}}$ [546-548]. Combined significance reaches $4 \sigma$ deviation from the SM [549]. The LHCb experiment has also measured $R_{K^{(*)}}$ LFU ratios, related to the neutral-current process $b \rightarrow s l l$, and found them to be lower than expected in the SM. While $R_{K}$ was measured in a single kinematical region, $q^{2} \in[1.1,6] \mathrm{GeV}^{2}[12], R_{K^{*}}$ was measured also in the ultra-low region $q^{2} \in[0.045,1.1] \mathrm{GeV}^{2}$ [550]. Each of the $R_{K^{(*)}}$ measurements is $\sim 2.5 \sigma$ below the $\mathrm{SM}$ prediction level [551, 552], and furthermore, there are discrepancies in $b \rightarrow$ sll driven decays that are coherent with the deviation in $R_{K}^{(*)}$ if there is $\sim 20 \%$ reduction in the vector Wilson coefficient $C_{9}[553,554]$.

Light leptoquarks are prime candidates to explain one or both of those puzzles. For the $R_{D^{(*)}}$ the effective Lagrangian contains four relevant operators:

$$
\begin{aligned}
\mathcal{L}_{\mathrm{eff}}^{b \rightarrow c \tau} \bar{\nu}_{\tau}=-\frac{4 G_{F}}{\sqrt{2}} & V_{c b}\left[\left(1+g_{V_{L}}\right)\left(\bar{c}_{L} \gamma_{\mu} b_{L}\right)\left(\bar{\tau}_{L} \gamma^{\mu} v_{\tau L}\right)\right. \\
& +g_{S_{L}}(\mu)\left(\bar{c}_{R} b_{L}\right)\left(\bar{\tau}_{R} v_{\tau L}\right)+g_{S_{R}}(\mu)\left(\bar{c}_{L} b_{R}\right)\left(\bar{\tau}_{R} v_{\tau L}\right) \\
& \left.+g_{T}(\mu)\left(\bar{c}_{R} \sigma_{\mu \nu} b_{L}\right)\left(\bar{\tau}_{R} \sigma^{\mu \nu} v_{\tau L}\right)\right] .
\end{aligned}
$$

Model independently it has been shown that $R_{D^{(*)}}$ can be explained either by rescaling the SM semileptonic operator $\left(g_{V_{L}}\right)$, by turning on $g_{T}$, or by particular combinations of scalar and tensor operators, $g_{S_{L}}= \pm 4 g_{T}$ operators, that arise in presence of a non-chiral LQ [555-558]. In order to address $R_{K^{(*)}}$ one has to modify the vector Wilson coefficient $C_{9}$ whereas the axial Wilson coefficient $C_{10}$ may also be present in the effective Lagrangian:

$$
\mathcal{H}_{\mathrm{eff}}^{b \rightarrow s \mu \mu}=-\frac{\alpha G_{F} V_{t b} V_{t s}^{*}}{\sqrt{2} \pi}\left(\bar{s}_{L} \gamma_{\mu} b_{L}\right)\left(\bar{\mu} \gamma^{\mu}\left(C_{9}+C_{10} \gamma^{5}\right) \mu .\right.
$$

Also purely left-handed scenarios with $C_{9}=-C_{10} \approx$ -0.6 , which are characteristic of LQ weak-singlet or triplet exchange, are in good agreement with $R_{K^{(*)}}$ and the global fit of $b \rightarrow s \ell \ell$. Such left-handed leptoquark solutions have been put forward: triplet scalar $S_{3}$, singlet vector $U_{1}$, triplet vector $U_{3}$ [557, 558]. For loop-level explanation of $R_{K^{(*)}}$ one can also invoke singlet $S_{1}$ [559] or doublet $R_{2}$ [560], but at the price of large couplings. There are several proposals with scalar leptoquarks that address $R_{K^{(*)}}$ and/or $R_{D^{(*)}}$ [559, 561-566], some in the context of unified theories such as SU(5) [567-569], left-right symmetry [570], Pati-Salam [571, 572], SO(10) [573] and others [574]. Recently it was realized that a singlet vector leptoquark $U_{1}^{\mu}(3,1,2 / 3)$ generates left-handed interactions and partially resolves both LFU puzzles, in many UV frameworks [575-577], including three-flavor extensions of the Pati-Salam model [578-584].

Finally, moderately large leptoquark couplings dictated by the above LFU anomalies can be also observed in processes with virtual LQ exchange, typically in the $t$-channel, resulting in a final state with at least one charged lepton. Inspired by the abovementioned LFU anomalies, processes with final state leptons have been studied, which can probe LQ scenarios for LFU violation observed in $B$ meson decays [585-588]. In this case, LQ cannot be produced on-shell and the sensitivity does not deteriorate abruptly with rising LQ mass. Instead there is a smooth transition to the effective theory picture, where heavy LQ is integrated out. Thus, among the LQ induced processes the $t$-channel has the best mass reach for LQs and it is thus complementary to pair and single production [489, 490]. Another recent set of observables at the LHC, targeting the LQ scenarios that are well suited to explain the LFU anomalies $R_{D^{(*)}}$, are the searches with a single $\tau$ lepton in the final state $[589]^{17}$. Third generation leptoquarks could also be probed in $t \bar{t}$ final states [591].

\subsection{Lepton Flavor Violation and Dipole Moments}

In the SM with massive neutrinos, lepton flavor violation (LFV) can occur via the mixing in the neutrino sector. It is, however, heavily suppressed due to the GIM mechanism [592], as the rate depends on the neutrino masses resulting in an unobservable prediction of order $10^{-55}$ [593]. Extensions of the SM modify this prediction by introducing additional sources of lepton flavor violation [594-596]. New physics can then be probed by testing the deviations of certain lepton flavor violating processes with respect to the experimental limits.

Charged lepton flavor violating processes are typically of three types: $l_{\alpha}^{-} \rightarrow l_{\beta}^{-} \gamma, l_{\alpha}^{-} \rightarrow l_{\beta}^{-} l_{\gamma}^{-} l_{\delta}^{+}$(with $\alpha \neq \beta$ ) and $\mu-e$ conversion in nuclei [597]. One-loop contributions to the first two processes can occur through a dipole and box diagrams as depicted in Figure 16, with a scalar or vector mediator(s) $X\left(X^{\prime}\right)$ and a SM or exotic fermion(s) $f\left(f^{\prime}\right)$ running in the loop. Contributions to $\mu-e$ conversion follows from the penguin (center) and box (right) diagrams with $l_{\alpha}=\mu, l_{\beta}=e$ and $l_{\gamma, \delta}=q$.

Unified theories often contain a number of exotic states capable of fulfilling the role of $X$ and $f$ in Figure 16, violating lepton flavor either by interactions between the leptons and mediators or by mixing in the leptonic sector. The latter case is realized in GUT models with heavy neutrinos (c.f. left-right models in section 4.1), where the mixing between active and sterile neutrinos enhances the LFV contribution [598-600]. The contribution to the branching ratios of the most constraining LFV processes, $\mu \rightarrow e \gamma, \mu \rightarrow$ eee and $\mu-e$ conversion, in these models, with heavy neutrinos of mass $M_{N_{I}}$, active-sterile mixing $\Theta_{\alpha I}$, a right-handed gauge boson $W_{R}$ and left and righthanded scalar triplets $\delta_{L}$ and $\delta_{R}$, can be written as [601], [602], and [174]

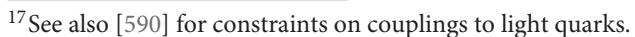



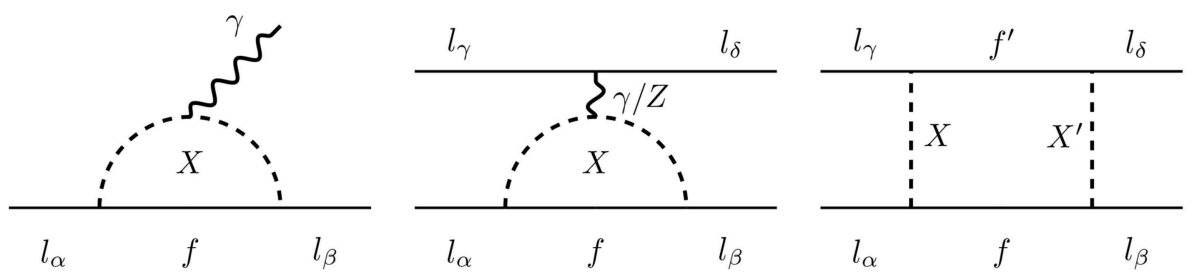

FIGURE 16 | Diagrams contributing to LFV processes, $I_{\alpha}^{-} \rightarrow I_{\beta}^{-} \gamma$ (left) and $I_{\alpha}^{-} \rightarrow I_{\beta}^{-} I_{\gamma}^{-} I_{\delta}^{+}$(center and right) with $X$ and $X^{\prime}$ scalar or vector mediators and $f$ and $f^{\prime}$ fermions.

$$
\begin{aligned}
B R(\mu \rightarrow e \gamma) & \sim 1.5 \times 10^{-7}\left|\Theta_{e I}^{*} \Theta_{\mu I}\right|^{2}\left(\frac{g_{R}}{g_{L}}\right)^{4}\left(\frac{m_{N_{I}}}{m_{W_{R}}}\right)^{4}\left(\frac{1 \mathrm{TeV}}{M_{W_{R}}}\right)^{4}, \\
B R(\mu \rightarrow e e e) & \sim \frac{1}{2}\left|\Theta_{e I}^{*} \Theta_{\mu I}\right|^{2}\left|\Theta_{e I}\right|^{4}\left(\frac{g_{R}}{g_{L}}\right)^{4}\left(\frac{m_{N_{I}}}{m_{W_{R}}}\right)^{4}\left(\frac{M_{W_{R}}^{4}}{M_{\delta_{R}}^{4}}+\frac{M_{W_{R}}^{4}}{M_{\delta_{L}}^{4}}\right), \\
R^{N}(\mu-e) & \sim 0.73 \times 10^{-9} X_{N}\left|\Theta_{e I}^{*} \Theta_{\mu I}\right|^{2}\left(\frac{g_{R}}{g_{L}}\right)^{4}\left(\frac{m_{N_{I}}}{m_{W_{R}}}\right)^{4}\left(\frac{1 \mathrm{TeV}}{M_{\delta_{R}}}\right)^{4}\left(\log \frac{m_{\delta_{R}}^{2}}{m_{\mu}^{2}}\right)^{2} .
\end{aligned}
$$

In supersymmetric GUTs there are many possible sources of lepton flavor violation, parametrised by the mixing in the slepton sector of the MSSM, which has contributions to LFV processes of the type [603]

$$
B R\left(l_{\alpha} \rightarrow l_{\beta} \gamma\right) \approx \frac{48 \pi^{3} \alpha_{\mathrm{em}}}{G_{F}^{2}} \frac{\left|\left(m_{\tilde{L}}^{2}\right)_{i j}\right|^{2}+\left|\left(m_{\tilde{e}}^{2}\right)_{i j}\right|^{2}}{M_{S U S Y}^{8}} B R\left(l_{\alpha} \rightarrow l_{\beta} v_{\alpha} \bar{v}_{\beta}\right) .
$$

Off-diagonal entries in the slepton mass matrices can be the result of non-minimal flavor violating interactions or non-canonical Yukawa textures at the GUT scale, where the soft masses are supposed to unify $[604,605]$. In addition, slepton mixing can be induced in minimal flavor violating (MFV) SUSY via seesaw mechanisms [606-612] or, for Yukawa-unified theories (see section 3.3), it can depend on the CKM matrix at the GUT scale [596]. LFV contributions can also arise in SUSY models where $R$-parity is violated, explicitly or spontaneously, with interaction terms of the type $l_{i} l_{j} \tilde{v}_{k}$ that induce tree-level contributions to $l \rightarrow l l l$ decays and $\mu \rightarrow e$ conversion, as well as new dipole contributions to $l \rightarrow l \gamma$ [613-617].

The anomalous electric, $d_{i}$, and magnetic, $a_{i}$, dipole moments of quarks and leptons follow from processes identical to the diagram on the left in Figure 16, where $l_{\alpha}$ and $l_{\beta}$ have the same flavor. Hence contributions from heavy states running in the loops can have a strong effect that can be tested experimentally. As with LFV, the SM contribution to electric dipole moments (EDMs) is tiny, as it is proportional to the CP-violation phase in the CKM matrix [618, 619]. EDMs have not been observed experimentally, so deviations from the SM prediction due to CPviolation in other sectors is strongly constrained [620]. Other sources of CP violation can appear in neutrino mixing [619], phases in fermion-sfermion couplings [621] or extended Higgs sectors [622]. Anomalous magnetic moments (AMM), on the other hand, have been measured with extreme accuracy. In fact, the precision of both the experimental measurement and theoretical prediction for $a_{\mu}$ has shown a discrepancy of more than 3 standard deviations [34]. New physics contributions have been shown to resolve that tension, particularly in the context of supersymmetry [623].

In the presence of light leptoquarks anomalous dipole moments of leptons or quarks are one-loop processes [480]. A special feature of non-chiral leptoquarks, such as $R_{2}$ with couplings (61), is that both $l_{i j}$ and $r_{i j}$ are non-zero in the interaction Lagrangian $\bar{q}^{i}\left[l_{i j} P_{R}+r_{i j} P_{L}\right] \ell^{j} \phi$ which then leads to the anomalous moment of the muon:

$$
\begin{array}{r}
a_{\mu}=-\frac{3 m_{\mu}^{2}}{8 \pi^{2} m_{\phi}^{2}} \sum_{q}\left[\left(\left|l_{q \mu}\right|^{2}+\left|r_{q \mu}\right|^{2}\right)\left(Q_{\phi} / 4-1 / 6\right)\right. \\
\left.-\frac{m_{q}}{m_{\mu}} \log \frac{m_{q}^{2}}{m_{\phi}^{2}} \operatorname{Re}\left(r_{q \mu}^{*} l_{q \mu}\right)\left(Q_{\phi}-1\right)\right],
\end{array}
$$

where $m_{\phi}$ and $Q_{\phi}$ are the charge and mass of the leptoquark and $q$ is the flavor of the quark in the loop (see the leftmost diagram in Figure 16). Shown is the leading order contribution in $m_{q}$. The first term increases $a_{\mu}$ only when $Q_{\phi}>2 / 3$ and it is present for all scalar LQ states that couple to a muon. The second term is relevant for non-chiral LQs and it is chirally enhanced by $m_{q} / m_{\ell}$, possibly leading to large effects with moderate couplings to $b$ or $t$ quark. Furthermore, the sign of the non-chiral term is adjustable. On the other hand, the same mechanism also enhances dipole LFV transitions, e.g., $\mu \rightarrow e \gamma, \tau \rightarrow \mu \gamma$ [560]. Non-chiral LQs may also generate quark or lepton electric dipole moments at 1-loop [624, 625].

Whichever the mechanism, it is clear that LFV and anomalous dipole moments are predicted by many GUT models, with varying strengths for different processes. The full list of processes and their current experimental upper bounds and measurements can be seen in Table 2, where the experiments that have studied the processes are detailed. Furthermore, new experiments are 
TABLE 2 | Upper bounds at 90\% C.L on LFV processes and EDMs, and measurements of AMMs, along with the experiments that provided them.

\begin{tabular}{|c|c|c|}
\hline Process & Branch. Frac. & Reference \\
\hline$\mu^{-} \rightarrow e^{-} \gamma$ & $4.2 \times 10^{-13}$ & MEG [626] \\
\hline$\tau^{-} \rightarrow e^{-} \gamma$ & $5.4 \times 10^{-8}$ & BaBar [627], Belle [628] \\
\hline$\tau^{-} \rightarrow \mu^{-} \gamma$ & $5.0 \times 10^{-8}$ & BaBar [627], Belle [628] \\
\hline$\mu^{-} \rightarrow e^{-} e^{-} e^{+}$ & $1.0 \times 10^{-12}$ & SINDRUM [629] \\
\hline$\tau^{-} \rightarrow e^{-} e^{-} e^{+}$ & $1.4 \times 10^{-8}$ & BaBar [630], Belle [631] \\
\hline$\tau^{-} \rightarrow \mu^{-} \mu^{-} \mu^{+}$ & $1.2 \times 10^{-8}$ & $\begin{array}{l}\text { ATLAS [632], BaBar [630], } \\
\text { Belle [631], LHCb [633] }\end{array}$ \\
\hline$\tau^{-} \rightarrow \mu^{-} e^{-} e^{+}$ & $1.1 \times 10^{-8}$ & BaBar [630], Belle [631] \\
\hline$\tau^{-} \rightarrow e^{-} e^{-} \mu^{+}$ & $0.84 \times 10^{-8}$ & BaBar [630], Belle [631] \\
\hline$\tau^{-} \rightarrow e^{-} \mu^{-} \mu^{+}$ & $1.6 \times 10^{-8}$ & BaBar [630], Belle [631] \\
\hline$\tau^{-} \rightarrow \mu^{-} \mu^{-} e^{+}$ & $0.98 \times 10^{-8}$ & BaBar [630], Belle [631] \\
\hline$\mu-e(T i)$ & $1.7 \times 10^{-12}$ & SINDRUM II [634] \\
\hline$\mu-e(\mathrm{~Pb})$ & $4.6 \times 10^{-11}$ & SINDRUM II [635] \\
\hline$\mu-e(\mathrm{Au})$ & $8.0 \times 10^{-13}$ & SINDRUM II [636] \\
\hline$d_{e}$ & $1.1 \times 10^{-29} \mathrm{e} \mathrm{cm}$ & ACME ॥ [637] \\
\hline$d_{\mu}$ & $1.9 \times 10^{-19} \mathrm{e} \mathrm{cm}$ & Muon g-2 [638] \\
\hline$d_{\tau}$ & $4.5 \times 10^{-17} \mathrm{e} \mathrm{cm}$ & Belle [639] \\
\hline$a_{e}\left(10^{-13}\right)$ & $11596521809.1 \pm 2.6$ & {$[640]$} \\
\hline$a_{\mu}\left(10^{-10}\right)$ & $11659208.9 \pm 8.7$ & Muon g-2 [641] \\
\hline
\end{tabular}

The HFLAV average is quoted for limits by different experiments [549].

being developed at this moment that will attempt to improve the limits on processes like $\mu \rightarrow$ eee (Mu3e [642]) and $\mu-e$ conversion (COMET [643], Mu2e [644]), with projected limits up to four orders of magnitude stronger than previous studies. Additionally, a new measurement of $a_{\mu}$ has been performed by the Muon g-2 experiment and it is expected to be released soon [645], which may confirm the deviation observed before, and thus further motivate the need of new physics.

\subsection{Neutrinoless Double Beta Decay}

This rare nuclear process corresponding to a simultaneous conversion of two neutrons to two protons and two electrons within the nucleus [646] is of great interest for particle physics, as it clearly does not conserve lepton number, and thus violates the corresponding accidental Abelian global symmetry of the SM. Consequently, a strong experimental effort is being made to observe this unique process. Unfortunately, its observation will be very difficult, as $0 \nu \beta \beta$ decay is expected to be extremely rare. There is a number of experiments, some in operation, other being constructed or planned, attempting to measure the decay. An overview of the major collaborations is shown in Table 3. The current experimental lower limits on its half-life are around $10^{26}$ years $[647,648]$ and the future searches should reach sensitivities by one or two orders of magnitude higher.

As can be shown, the existence of $0 \nu \beta \beta$ decay implies the Majorana nature of neutrinos (and vice versa) [649-651] and as such it represents one of the best probes of this BSM hypothesis. Therefore, in GUT models allowing for Majorana neutrino mass generation $0 \nu \beta \beta$ decay can be in principle always triggered. This, however, does not say anything about the underlying mechanism and the resulting $0 \nu \beta \beta$ decay rate. The prominent standard (mass) mechanism of $0 \nu \beta \beta$ decay assumes a light Majorana neutrino exchange between the two beta-decaying
TABLE 3 | An overview of both current and future major $0 v \beta \beta$ decay searches.

\begin{tabular}{lcccc}
\hline Experiment & Isotope & Status & $\boldsymbol{M}[\mathbf{k g}]$ & $\boldsymbol{T}_{\mathbf{1 / 2}}^{\mathbf{0} \boldsymbol{\beta} \boldsymbol{\beta} \boldsymbol{\beta}}$ limit [y] \\
\hline CUORE & ${ }^{130} \mathrm{Te}$ & running & 200 & $\left(3.5 \times 10^{26}\right)$ \\
EXO-200 & ${ }^{136} \mathrm{Xe}$ & running & 110 & $1.1 \times 10^{25}$ \\
nEXO & ${ }^{136} \mathrm{Xe}$ & R\&D & 5000 & $\left(10^{27}-10^{28}\right)$ \\
GERDA & ${ }^{76} \mathrm{Ge}$ & running & 21.6 & $5.3 \times 10^{25}$ \\
& & in progress & 40 & $\left(\sim 10^{26}\right)$ \\
KamLAND-Zen & ${ }^{136} \mathrm{Xe}$ & running & 383 & $1.1 \times 10^{26}$ \\
& & in progress & 600 & $\left(2 \times 10^{26}\right)$ \\
LEGEND & ${ }^{76} \mathrm{Ge}$ & R\&D & 200 & $\left(\sim 10^{27}\right)$ \\
& & R\&D & 1000 & $\left(\sim 10^{28}\right)$ \\
Majorana Dem. & ${ }^{76 s} \mathrm{Ge}$ & running & 44.1 & $1.9 \times 10^{25}$ \\
NEXT & ${ }^{136} \mathrm{Xe}$ & in progress (demo) & 100 & $\left(5.9 \times 10^{25}\right)$ \\
SNO+ & ${ }^{130} \mathrm{Te}$ & in progress & 1300 & $\left(2 \times 10^{26}\right)$ \\
SuperNEMO & ${ }^{82} \mathrm{Se}\left({ }^{150} \mathrm{Nd}\right)$ & in progress (demo) & 100 & $\left(\sim 10^{26}\right)$ \\
\hline
\end{tabular}

For each experiment the following information is shown: used isotope, operational status, the deployed mass $M$ of the isotope in question and the measured or expected (for experiment in preparation these values are shown in parentheses) sensitivity $T_{1 / 2}^{0 v \beta \beta}$. For some experiments (GERDA, KamLAND-Zen, LEGEND) characteristics of more stages of development are given. In case of SuperNEMO, the primary isotope to be tested is ${ }^{82} \mathrm{Se}$ and in the future the measurement will be repeated with a ${ }^{150} \mathrm{Nd}$ source.

neutrons. In the SM with light massive neutrinos this process can be depicted as shown in Figure 17, left. Besides the standard scenario a number of non-standard mechanisms triggering $0 v \beta \beta$ decay can be constructed. The effective treatment of these exotic mechanisms can be conveniently employed (see e.g., [652-656]).

As for the UV-complete $0 v \beta \beta$ decay mechanisms, a variety of interesting ones can be constructed within GUTs. For instance, in the left-right symmetric models (where, of course, the standard light neutrino exchange is available) one can think of several exotic mechanisms involving exchange of heavy neutrino as well as light and heavy $W$ vector bosons [657]. In the simplest exotic case the light neutrino exchange is substituted by a heavy righthanded neutrino exchange, which means that the involved vector currents and emitted electrons must be also right-handed. Due to the large mass of the propagating neutrino, the interaction can be considered to be contact and we refer to this contribution as to short-range mechanism. Since the right-handed currents are present in left-right symmetric models, it is also possible to draw $0 \nu \beta \beta$ decay mechanisms, in which the neutrino exchange does not violate chirality. This means that the contribution is not proportional to the neutrino mass and the two outgoing electrons are of opposite chiralities. A possible mechanism of this type is depicted in Figure 17, right. As apparent, the diagram involves one right-handed and one left-handed vector current and since the light neutrino propagator is present, one refers to this contribution as to a long-range $0 \nu \beta \beta$ decay mechanism.

Leptoquarks, particles appearing prominently in GUTs, can also trigger non-standard $0 v \beta \beta$ decay contributions. It has been described that this is the case, when different leptoquark multiplets mix via a possible leptoquark-Higgs coupling violating lepton number $[658,659]$. Diagrams of this type of contributions to $0 v \beta \beta$ decay are shown in Figure 18. The specific helicity structure of the effective four-fermion interaction leads to the fact that this contribution can dominate over the standard 

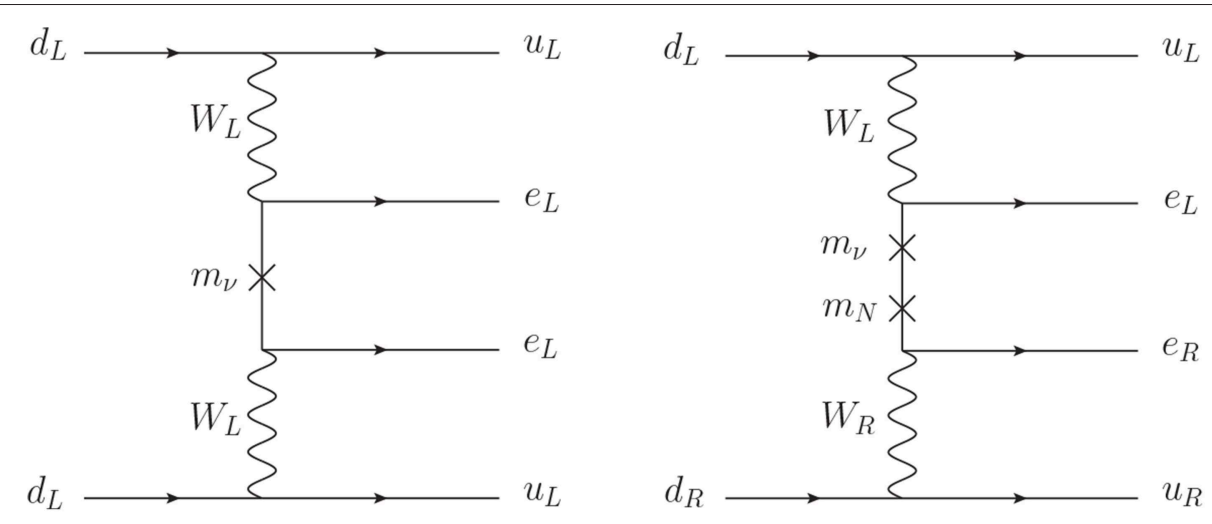

FIGURE 17 | The standard mass mechanism of $0 v \beta \beta$ decay (left) and a non-standard contribution that can be constructed in the left-right symmetric models using vector currents of opposite chiralities (right).
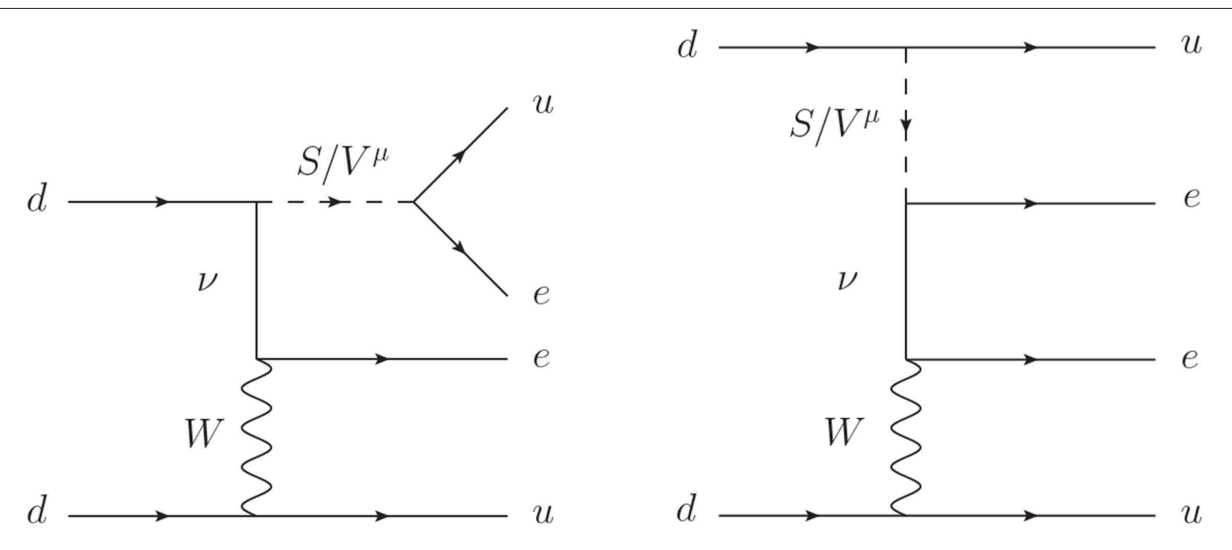

FIGURE 18 | The exotic $0 \nu \beta \beta$ decay mechanisms involving scalar or vector leptoquarks $S, V^{\mu}$.

mass mechanism. The current lower limits on $0 v \beta \beta$ decay half-life then allow to derive the bounds on corresponding leptoquark parameters.

Neutrinoless double beta decay can be triggered also in supersymmetric theories aspiring for grand unification. In the simplest case, if the MSSM with broken $R$ parity is considered, $0 \nu \beta \beta$ decay diagrams involving $R$-parity-violating couplings and supersymmetric mediators can be drawn [660-664]. An example of a supersymmetric $0 \nu \beta \beta$ decay mechanism is depicted in Figure 19. Again, from non-observation of $0 v \beta \beta$ decay it is possible to derive limits on the unknown model parameters.

\section{OUTLOOK AND FUTURE PROSPECTS FOR GUTS}

Among the vast landscape of theories beyond the Standard Model, Grand Unified Theories stand out as appealing candidates. As we have seen, GUTs are a collection of ideas from group theory, supersymmetry, neutrino physics, flavor physics and more, which positions them as some of the most complete and attractive theories in the literature. Indeed they are among the few BSM theories capable of simultaneously affecting the

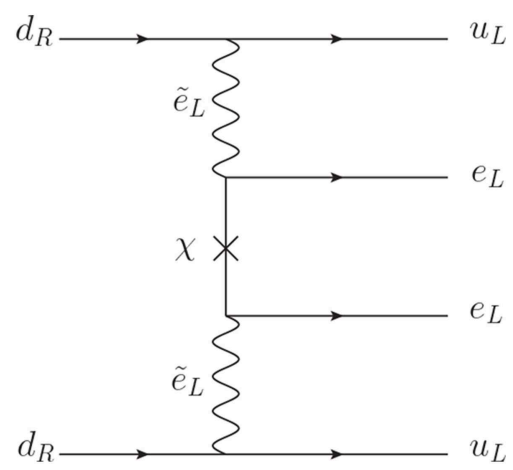

FIGURE 19 | An example of a $0 \nu \beta \beta$ decay mechanism that can be triggered in R-parity-violating MSSM.

highest energy scales, influencing the cosmology of the early Universe, and the low energies, within reach of colliders and terrestrial experiments.

Throughout this review article we have provided a rough sketch of the status of GUTs and some of the associated research 
in the field. We have described the basic principles behind them and their first appearance in the world of particle physics in the late 70s. A lot of effort was spent in the years after and many of the greatest models were designed at that time. Research in GUTs has continued since, focusing either on specific topics within and alongside them, or on particular models that compiled a few advances in the field. We have thus summarized a non-exhaustive selection of topics and models that are at the forefront of research in unified theories, aiming to provide an overview of the current state of the art.

We are fortunate enough to live in a time where experimental searches are abundant and they cover a rather vast range of fronts. The most cutting-edge technologies have been and are being developed to push the boundaries of our current understanding of particle physics and cosmology. Grand unified theories are and will be put under the microscope by many of these experimental advances, which will confirm, constrain or outright exclude some of the existing models.

The recent observation of gravitational wave signatures opens a new window into the history of the Universe, where events and phenomena that ocurred in the early Universe can be observed with gravitational wave detectors. Cosmic phase transitions associated with patterns of symmetry breaking in unified theories are such events, as they can be the source of stochastic gravitational waves that can be observed today. Transition temperatures above the EW scale, typically associated with the breaking of some intermediate step in a GUT model, can be studied by future gravitational wave experiments such as a LISA, the Einstein telescope, Kagra, the Cosmic Explorer, BBO and DEIGO. Also in the cosmological frontier, GUTs can have a serious impact on the inflationary epoch of the Universe, testable in measurements of the $\mathrm{CMB}$, and can contribute to the baryon asymmetry of the Universe, via baryo and leptogenesis.

At the time of writing we have reached the end of the second run of the LHC, with an outstanding recorded integrated luminosity of about $150 \mathrm{fb}^{-1}$. Analyses of the accumulated data, however, are still under way and they will probably spill well into the start run 3 in 2021. Many of the analyses already published have strong consequences for the predictions of unified theories, as are direct searches for supersymmetry, leptoquarks or other exotics at ATLAS and CMS. Upcoming results from ongoing and future analyses of the results from the LHC experiments may strengthen the bounds on light states as predicted by SUSY GUTs and other models, or they might show hints of the existence of new particles, whose relevance for GUTs would need to be determined. Upgraded versions of the LHC (HLLHC, VLHC or FCC) or other future colliders (ILC, CLIC) will

\section{REFERENCES}

1. Glashow S. Partial symmetries of weak interactions. Nucl.Phys. (1961) 22:579-88. doi: 10.1016/0029-5582(61)90469-2

2. Weinberg S. A model of leptons. Phys Rev Lett. (1967) 19:1264.

3. Salam A. Weak and electromagnetic interactions. Conf Proc C. (1968) 680519:367-77.

4. Aad G, Abajyan T, Abbott B, Abdallah J, Khalek SA, Abdelalim AA, et al. Observation of a new particle in the search for the Standard Model certainly boost this programme with increased accuracy and higher energies, which will further probe the low-hanging states predicted by GUTs.

Where colliders search for the low scale predictions of GUTs, precision experiments can explore the intermediate and high scales associated with unification. Nucleon decay limits are often among the strongest probes of fully unified theories and future experiments such as Hyper-Kamiokande and DUNE may set even stronger exclusion limits or perhaps measure signs of proton decay, which would be a smoking gun for GUTs. Furthermore, GUTs can provide contributions to a number of flavor and precision observables, such as LFU, LFV, EDM, AMM, or $0 v \beta \beta$, some of which are in tension with the SM. Confirmation of these flavor anomalies with more collected data by $\mathrm{LHCb}$ and other experiments would be undeniable evidence of the need for new physics models and GUTs are very well suited for that purpose.

To conclude, Grand Unified Theories are still at the vanguard of research in BSM models. They can explain many of the issues of the SM and can accommodate the recent results from the cosmological, precision and collider frontiers with relative ease. Contrary to "simplified" models, GUTs are complete theories that can simultaneously make a large number of testable predictions on the different fronts. Fortunately, these predictions can be explored by upcoming analyses and future experiments, which can set strong exclusion limits in a subset of GUT models. On a more optimistic note, any observation in, for instance, SUSY searches at colliders, gravitational waves signatures or proton decay will stack the odds in favor of some GUT models and will significantly shape the future of the research in particle physics.

\section{AUTHOR CONTRIBUTIONS}

TG has lead the development of this review. All other authors have contributed equally.

\section{ACKNOWLEDGMENTS}

The authors would like to thank P. Athron, F. Deppisch, A. Kvellestad, and Y. Zhang for helpful discussions. TG was partly funded by the Research Council of Norway under FRIPRO project number 230546/F20 and partly supported by the ARC Centre of Excellence for Particle Physics at the Tera-scale, grant CE110001004. NK was supported by the Slovenian Research Agency under the research core funding grant P1-0035 and No. J1-8137. TRIUMF receives federal funding via a contribution agreement with the National Research Council of Canada and the Natural Science and Engineering Research Council of Canada.
Higgs boson with the ATLAS detector at the LHC. Phys Lett. (2012) B716:1-29.

5. Chatrchyan S, Khachatryan V, Sirunyan AM, Tumasyan A, Adam W, Aguilo E, et al. Observation of a new boson at a mass of $125 \mathrm{GeV}$ with the CMS experiment at the LHC. Phys Lett. (2012) B716:30-61. doi: 10.1016/j.physletb. 2012.08.021

6. Higgs PW. Broken symmetries and the masses of gauge bosons. Phys Rev Lett. (1964) 13:508-9. 
7. Englert F, Brout R. Broken symmetry and the mass of gauge vector mesons. Phys Rev Lett. (1964) 13:321-3.

8. Guralnik GS, Hagen CR, Kibble TWB. Global conservation laws and massless particles. Phys Rev Lett. (1964) 13:585-7.

9. Degrassi G, Di Vita S, Elias-Miro J, Espinosa JR, Giudice GF, Isidori G, et al. Higgs mass and vacuum stability in the Standard Model at NNLO. J High Energy Phys. (2012) 08:098.

10. Fukuda $\mathrm{Y}$, Hayakawa T, Ichihara E, Inoue $\mathrm{K}$, Ishihara $\mathrm{K}$, Ishino H, et al. Evidence for oscillation of atmospheric neutrinos. Phys Rev Lett. (1998) 81:1562-7.

11. Ahmad QR, Allen RC, Andersen TC, Anglin JD, Buhler G, Barton JC, et al. Measurement of the rate of $v_{e}+d \rightarrow p+p+e^{-}$interactions produced by ${ }^{8} B$ solar neutrinos at the Sudbury Neutrino Observatory. Phys Rev Lett. (2001) 87:071301. doi: 10.1103/PhysRevLett.87.071301

12. Aaij R, Adeva B, Adinolfi M, Affolder A, Ajaltouni Z, Akar S, et al. Test of lepton universality using $B^{+} \rightarrow K^{+} \ell^{+} \ell^{-}$decays. Phys Rev Lett. (2014) 113:151601. doi: 10.1103/PhysRevLett.113.151601

13. Aaij R, Adeva B, Adinolfi M, Affolder A, Ajaltouni Z, Akar S, et al. Measurement of the ratio of branching fractions $\mathcal{B}\left(\bar{B}^{0} \rightarrow\right.$ $\left.D^{*+} \tau^{-} \bar{v}_{\tau}\right) / \mathcal{B}\left(\bar{B}^{0} \rightarrow D^{*+} \mu^{-} \bar{v}_{\mu}\right)$. Phys Rev Lett. (2015) 115:111803. doi: 10.1103/PhysRevLett.115.111803

14. Aaij R, Adeva B, Adinolfi M, Ajaltouni Z, Akar S, Albrecht J, et al. Measurement of the $B_{s}^{0} \rightarrow \mu^{+} \mu^{-}$branching fraction and effective lifetime and search for $B^{0} \rightarrow \mu^{+} \mu^{-}$decays. Phys Rev Lett. (2017) 118:191801. doi: 10.1103/PhysRevLett.118.191801

15. Georgi H, Glashow SL. Unity of all elementary-particle forces. Phys Rev Lett. (1974) 32:438-41.

16. Pati JC, Salam A. Lepton number as the fourth color. Phys Rev. (1974) D10:275-89.

17. Mohapatra RN, Pati JC. Left-right gauge symmetry and an isoconjugate model of CP violation. Phys Rev. (1975) D11:566-71.

18. Fritzsch $\mathrm{H}$, Minkowski P. Unified interaction of leptons and hadrons. Ann Phys. (1975) 93:193-266.

19. Georgi H. The state of the art-gauge theories. AIP Conf Proc. (1975) 23:57582.

20. Langacker P. Grand unified theories and proton decay. Phys Rept. (1981) $72: 185$.

21. Gonzalo Velasco T. Model Building and Phenomenology in Grand Unified Theories. University Coll. London (2015). Available online at: http://discovery.ucl.ac.uk/id/eprint/1471160

22. Raby S. Supersymmetric grand unified theories. Lect Notes Phys. (2017) 939:1-308. doi: 10.1007/978-3-319-55255-2

23. Nath P. High energy physics and cosmology at the unification frontier: opportunities and challenges in the coming years. Int J Mod Phys. (2018) A33:1830017. doi: 10.1142/S0217751X1830017X

24. Candelas P, Horowitz GT, Strominger A, Witten E. Vacuum configurations for superstrings. Nucl Phys. (1985) B258:46-74.

25. Witten E. Symmetry breaking patterns in superstring models. Nucl Phys. (1985) B258:75. doi: 10.1016/0550-3213(85)90603-0

26. Gursey F, Sikivie P. E(7) as a universal gauge group. Phys Rev Lett. (1976) 36:775.

27. Branco GC, Ferreira PM, Lavoura L, Rebelo MN, Sher M, Silva JP. Theory and phenomenology of two-Higgs-doublet models. Phys Rept. (2012) 516:1102.

28. Georgi H, Quinn HR, Weinberg S. Hierarchy of interactions in unified gauge theories. Phys Rev Lett. (1974) 33:451-4.

29. Buras AJ, Ellis JR, Gaillard MK, Nanopoulos DV. Aspects of the grand unification of strong, weak and electromagnetic interactions. Nucl Phys. (1978) B135:66-92.

30. Chanowitz MS, Ellis JR, Gaillard MK. The price of natural flavor conservation in neutral weak interactions. Nucl Phys. (1977) B128:506-36.

31. Georgi H, Jarlskog C. A new lepton-quark mass relation in a unified theory. Phys Lett. (1979) 86B:297-300.

32. Dimopoulos S, Georgi H. Softly broken supersymmetry and SU(5). Nucl Phys. (1981) B193:150-62.

33. Masiero A, Nanopoulos DV, Tamvakis K, Yanagida T. Naturally massless higgs doublets in supersymmetric SU(5). Phys Lett. (1982) 115B:380-4.
34. Tanabashi M, Hagiwara K, Hikasa K, Nakamura K, Sumino Y, Takahashi F, et al. Review of particle physics. Phys Rev. (2018) D98:030001. doi: 10.1088/1674-1137/40/10/100001

35. Grinstein B. A supersymmetric SU(5) gauge theory with no gauge hierarchy problem. Nucl Phys. (1982) B206:387.

36. Hisano J, Moroi T, Tobe K, Yanagida T. Suppression of proton decay in the missing partner model for supersymmetric SU(5) GUT. Phys Lett. (1995) B342:138-44.

37. Antusch S, de Medeiros Varzielas I, Maurer V, Sluka C, Spinrath M. Towards predictive flavour models in SUSY SU(5) GUTs with doublet-triplet splitting. J High Energy Phys. (2014) 09:141. doi: 10.1007/ JHEP09(2014)141

38. Dorsner I, Fileviez Perez P, Gonzalez Felipe R. Phenomenological and cosmological aspects of a minimal GUT scenario. Nucl Phys. (2006) B747:312-27. doi: 10.1016/j.nuclphysb.2006.05.006

39. Bajc B, Senjanovic G. Seesaw at LHC. J High Energy Phys. (2007) 08:014. doi: 10.1088/1126-6708/2007/08/014

40. Fileviez Perez P. Renormalizable adjoint SU(5). Phys Lett. (2007) B654:189_ 93. doi: 10.1016/j.physletb.2007.07.075

41. Zee A. A theory of lepton number violation, neutrino majorana mass, and oscillation. Phys Lett. (1980) 93B:389.

42. Fileviez Perez P, Murgui C. Renormalizable SU(5) unification. Phys Rev. D. (2016) 94:075014. doi: 10.1103/PhysRevD.94.075014

43. Abe K, Haga Y, Hayato Y, M Ikeda KI, Kameda J, et al. Search for proton decay via $p \rightarrow e^{+} \pi^{0}$ and $p \rightarrow \mu^{+} \pi^{0}$ in 0.31 megaton years exposure of the super-kamiokande water cherenkov detector. Phys Rev D. (2017) 95:012004. doi: 10.1103/PhysRevD.95.012004

44. Dorsner I, Fileviez Perez P. Unification without supersymmetry: neutrino mass, proton decay and light leptoquarks. Nucl Phys. (2005) B723:53-76. doi: 10.1016/j.nuclphysb.2005.06.016

45. Dorsner I, Fileviez Perez P. Unification versus proton decay in SU(5). Phys Lett. (2006) B642:248-52. doi: 10.1016/j.nuclphysb.2005.06.016

46. Fornal B, Grinstein B. Grand unified theory with a stable proton. (2018) Int J Mod Phys. A33:1844013. doi: 10.1142/S0217751X1844013X

47. Fileviez Pérez P, Gross A, Murgui C. Seesaw scale, unification, and proton decay. Phys Rev D. (2018) 98:035032. doi: 10.1103/PhysRevD.98.035032

48. Weinberg S. Implications of dynamical symmetry breaking. Phys Rev . (1976) D13:974-96. doi: 10.1103/PhysRevD.13.974

49. Martin SP. A supersymmetry primer. (1997) 18:1-98. doi: 10.1142/9789812839657 0001

50. Nanopoulos DV, Tamvakis K. SUSY GUTS: 4 - GUTS: 3. Phys Lett. (1982) 113B:151-8. doi: 10.1016/0370-2693(82)90413-0

51. Dorsner I, Fileviez Perez P, Rodrigo G. On unification and nucleon decay in supersymmetric grand unified theories based on SU(5). Phys Lett. (2007) B649:197-205. doi: 10.1016/j.physletb. 2007.03.062

52. 't Hooft G. Magnetic monopoles in unified gauge theories. Nucl Phys. (1974) B79:276-84. doi: 10.1016/0550-3213(74)90486-6

53. Witten E. Mass hierarchies in supersymmetric theories. Phys Lett. (1981) 105B:267. doi: 10.1016/0370-2693(81)90885-6

54. Dimopoulos S, Georgi H. Solution of the Gauge Hierarchy Problem. Phys Lett. (1982) 117B:287-90. doi: 10.1016/0370-2693(82)90720-1

55. De Rujula A, Georgi H, Glashow SL. FLAVOR GONIOMETRY BY PROTON DECAY. Phys Rev Lett. (1980) 45:413. doi: 10.1103/PhysRevLett.45.413

56. Barr SM. A new symmetry breaking pattern for $\mathrm{SO}(10)$ and proton decay. Phys Lett. (1982) B112:219. doi: 10.1016/0370-2693(82) 90966-2

57. Derendinger JP, Kim JE, Nanopoulos DV. Anti-SU(5). Phys Lett. (1984) B139:170. doi: 10.1016/0370-2693(84)91238-3

58. Antoniadis I, Ellis JR, Hagelin JS, Nanopoulos DV. Supersymmetric flipped SU(5) revitalized. Phys Lett. (1987) B194:231. doi: 10.1016/0370-2693(87)90533-8

59. Ellis JR, Hagelin JS, Kelley S, Nanopoulos DV. Aspects of the flipped unification of strong, weak and electromagnetic interactions. Nucl Phys. (1988) B311:1. doi: 10.1016/0550-3213(88)90141-1

60. Campbell BA, Ellis JR, Hagelin JS, Nanopoulos DV, Ticciati R. Flipped SU(5) from manifold compactification of the ten-dimensional heterotic string. Phys Lett. (1987) B198:200-4. doi: 10.1016/0370-2693(87)91496-1 
61. Antoniadis I, Ellis JR, Hagelin JS, Nanopoulos DV. GUT model building with fermionic four-dimensional strings. Phys Lett. (1988) B205:459-65. doi: 10.1016/0370-2693(88)90978-1

62. Senjanovic G, Mohapatra RN. Exact left-right symmetry and spontaneous violation of parity. Phys Rev. (1975) D12:1502. doi: 10.1103/PhysRevD.12.1502

63. Mohapatra RN, Pati JC. A natural left-right symmetry. Phys Rev. (1975) D11:2558. doi: 10.1103/PhysRevD.11.2558

64. Smirnov AD. The minimal quark-lepton symmetry model and the limit on Z-prime mass. Phys Lett. (1995) B346:297-302. doi: 10.1016/0370-2693(95)00015-D

65. Fileviez Perez P, Wise MB. Low scale quark-lepton unification. Phys Rev D. (2013) 88:057703. doi: 10.1103/PhysRevD.88.057703

66. Mohapatra RN, Senjanovic G. Neutrino mass and spontaneous parity violation. Phys Rev Lett. (1980) 44:912. doi: 10.1103/PhysRevLett.44.912

67. Mohapatra RN, Senjanovic G. Neutrino masses and mixings in gauge models with spontaneous parity violation. Phys Rev. (1981) D23:165. doi: 10.1103/PhysRevD.23.165

68. Mohapatra RN, Marshak RE. Local B-L symmetry of electroweak interactions, majorana neutrinos and neutron oscillations. Phys Rev Lett. (1980) 44:1316-9. doi: 10.1103/PhysRevLett.44.1316

69. Lindner M, Weiser M. Gauge coupling unification in left-right symmetric models. Phys Lett. (1996) B383:405-14. doi: 10.1016/0370 -2693(96)00775-7

70. Aulakh CS, Bajc B, Melfo A, Rasin A, Senjanovic G. SO(10) theory of R-parity and neutrino mass. Nucl Phys. (2001) B597:89-109. doi: 10.1016/S0550-3213(00)00721-5

71. Senjanovic G. Spontaneous breakdown of parity in a class of gauge theories. Nucl Phys. (1979) B153:334-64. doi: 10.1016/0550-3213(79)90604-7

72. Antoniadis I, Leontaris GK. A SUPERSYMMETRIC SU(4) x O(4) MODEL. Phys Lett. (1989) B216:333-5. doi: 10.1016/0370-2693(89)91125-8

73. Kuchimanchi R, Mohapatra RN. No parity violation without R-parity violation. Phys Rev. (1993) D48:4352-60. doi: 10.1103/PhysRevD.48.4352

74. Cvetic M. Effects of the parity-odd singlet on the $N=1$ supergravity theory within the left-right symmetric model. Phys Lett. (1985) 164B:55-60. doi: 10.1016/0370-2693(85)90030-9

75. Aulakh CS, Benakli K, Senjanovic G. Reconciling supersymmetry and left-right symmetry. Phys Rev Lett. (1997) 79:2188-91. doi: 10.1103/PhysRevLett.79.2188

76. Aulakh CS, Melfo A, Senjanovic G. Minimal supersymmetric left-right model. Phys Rev. (1998) D57:4174-8. doi: 10.1103/PhysRevD.57.4174

77. Aulakh CS, Melfo A, Rasin A, Senjanovic G. Supersymmetry and large scale left-right symmetry. Phys Rev. (1998) D58:115007. doi: 10.1103/PhysRevD.58.115007

78. Georgi H, Glashow SL. Gauge theories without anomalies. Phys Rev. (1972) D6:429. doi: 10.1103/PhysRevD.6.429

79. Adler SL. Axial vector vertex in spinor electrodynamics. Phys Rev. (1969) 177:2426-38. doi: 10.1103/PhysRev.177.2426

80. Mohapatra RN, Sakita B. $\mathrm{SO}(2 \mathrm{n})$ grand unification in an $\mathrm{SU}(\mathrm{N})$ basis. Phys Rev. (1980) D21:1062. doi: 10.1103/PhysRevD.21.1062

81. Bertolini S, Di Luzio L, Malinsky M. The quantum vacuum of the minimal SO(10) GUT. J Phys Conf Ser. (2010) 259:012098. doi: 10.1088/1742-6596/259/1/012098

82. Bertolini S, Di Luzio L, Malinsky M. Seesaw scale in the minimal renormalizable SO(10) grand unification. Phys Rev. (2012) D85:095014. doi: 10.1103/PhysRevD.85.095014

83. Bertolini S, Di Luzio L, Malinsky M. Light color octet scalars in the minimal SO(10) grand unification. Phys Rev. (2013) D87:085020. doi: 10.1103/PhysRevD.87.085020

84. Kolesova H, Malinsky M. Proton lifetime in the minimal SO(10) GUT and its implications for the LHC. Phys Rev. (2014) D90:115001. doi: 10.1103/PhysRevD.90.115001

85. Graf L, Malinsky M, Mede T, Susic V. One-loop pseudo-Goldstone masses in the minimal SO(10) Higgs model. Phys Rev D. (2017) 95:075007. doi: 10.1103/PhysRevD.95.075007

86. Bertolini S, Schwetz T, Malinsky M. Fermion masses and mixings in SO(10) models and the neutrino challenge to SUSY GUTs. Phys Rev. (2006) D73:115012. doi: 10.1103/PhysRevD.73.115012
87. Aulakh CS, Garg SK. MSGUT : From bloom to doom. Nucl Phys. (2006) B757:47-78. doi: 10.1016/j.nuclphysb.2006.07.030

88. Aulakh CS, Girdhar A. SO(10) a la Pati-Salam. Int J Mod Phys. (2005) A20:865-94. doi: 10.1142/S0217751X0502001X

89. Anastaze G, Derendinger JP, Buccella F. INTERMEDIATE SYMMETRIES IN THE SO(10) MODEL WITH $(16+16)+45$ HIGGSES. $Z$ Phys. (1983) C20:269-73. doi: 10.1007/BF01574861

90. Clark TE, Kuo TK, Nakagawa N. A SO(10) SUPERSYMMETRIC GRAND UNIFIED THEORY. Phys Lett. (1982) 115B:26-8. doi: 10.1016/0370-2693(82)90507-X

91. Aulakh CS, Mohapatra RN. Implications of supersymmetric SO(10) grand unification. Phys Rev. (1983) D28:217. doi: 10.1103/PhysRevD.28.217

92. Tamvakis K. FLIPPED SO(10). Phys Lett. (1988) B201:95-100. doi: 10.1016/0370-2693(88)90087-1

93. Reig M, Valle JWF, Vaquera-Araujo CA, Wilczek F. A Model of Comprehensive Unification. Phys Lett. (2017) B774:667-70. doi: 10.1016/j.physletb.2017.10.038

94. Langacker P, Wang J. U(1)-prime symmetry breaking in supersymmetric E(6) models. Phys Rev. (1998) D58:115010. doi: 10.1103/PhysRevD.58.115010

95. Gursey F, Ramond P, Sikivie P. A universal gauge theory model based on E6. Phys Lett. (1976) 60B:177-80. doi: 10.1016/0370-2693(76)90417-2

96. Achiman Y, Stech B. Quark lepton symmetry and mass scales in an E6 unified gauge model. Phys Lett. (1978) 77B:389-93. doi: 10.1016/0370-2693(78)90584-1

97. Shafi Q. E(6) as a unifying gauge symmetry. Phys Lett. (1978) 79B:301-3. doi: 10.1016/0370-2693(78)90248-4

98. Mohapatra RN, Valle JWF. Neutrino mass and baryon number nonconservation in superstring models. Phys Rev. (1986) D34:1642. doi: 10.1103/PhysRevD.34.1642

99. Buccella F, Miele G. SO(10) from supersymmetric E(6). Phys Lett. (1987) B189:115-7. doi: 10.1016/0370-2693(87)91280-9

100. Hosotani Y. Dynamical mass generation by compact extra dimensions. Phys Lett. (1983) 126B:309-13. doi: 10.1016/0370-2693(83)90170-3

101. Hewett JL, Rizzo TG. Low-energy phenomenology of superstring inspired E(6) models. Phys Rept. (1989) 183:193. doi: 10.1016/0370-1573(89) 90071-9

102. London D, Rosner JL. Extra gauge bosons in E(6). Phys Rev. (1986) D34:1530. doi: 10.1103/PhysRevD.34.1530

103. Dimopoulos S, Raby S, Wilczek F. Supersymmetry and the scale of unification. Phys Rev. (1981) D24:1681-3. doi: 10.1103/PhysRevD.24.1681

104. Witten E. Dynamical breaking of supersymmetry. Nucl Phys. (1981) B188:513. doi: 10.1016/0550-3213(81)90006-7

105. Ibanez LE, Ross GG. SU(2)-L x U(1) symmetry breaking as a radiative effect of supersymmetry breaking in guts. Phys Lett. (1982) 110B:215-20. doi: 10.1016/0370-2693(82)91239-4

106. Ellis JR, Nanopoulos DV, Tamvakis K. Grand unification in simple supergravity. Phys Lett. (1983) 121B:123-9. doi: 10.1016/0370-2693(83)90900-0

107. Farrar GR, Fayet P. Phenomenology of the production, decay, and detection of new hadronic states associated with supersymmetry. Phys Lett. (1978) 76B:575-9. doi: 10.1016/0370-2693(78)90858-4

108. Ellis JR, Hagelin JS, Nanopoulos DV, Olive KA, Srednicki M. Supersymmetric relics from the big bang. Nucl Phys. (1984) B238:453-76. doi: 10.1016/0550-3213(84)90461-9

109. Jungman G, Kamionkowski M, Griest K. Supersymmetric dark matter. Phys Rept. (1996) 267:195-373. doi: 10.1016/0370-1573(95) 00058-5

110. Ellis JR, Kelley S, Nanopoulos DV. Probing the desert using gauge coupling unification. Phys Lett. (1991) B260:131-7. doi: 10.1016/0370-2693(91)90980-5

111. Martin SP, Vaughn MT. Two loop renormalization group equations for soft supersymmetry breaking couplings. Phys Rev. (1994) D50:2282. doi: 10.1103/PhysRevD.50.2282

112. Hall LJ. Grand unification of effective gauge theories. Nucl Phys. (1981) B178:75-124. doi: 10.1016/0550-3213(81)90498-3

113. Weinberg S. Effective gauge theories. Phys Lett. (1980) 91B:51-5. doi: 10.1016/0370-2693(80)90660-7 
114. Giudice GF, Romanino A. Split supersymmetry. Nucl Phys. (2004) B699:6589. doi: 10.1016/j.nuclphysb.2004.08.001

115. Arkani-Hamed N, Dimopoulos S. Supersymmetric unification without low energy supersymmetry and signatures for fine-tuning at the LHC. J High Energy Phys. (2005) 06:073. doi: 10.1088/1126-6708/2005/06/073

116. Athron P, Balázs C, Buckley A, Cornell JM, Danninger M, Farmer B, et al. Combined collider constraints on neutralinos and charginos. (2018). doi: 10.1140/epjc/s10052-019-6837-x

117. Ellis SAR, Wells JD. Visualizing gauge unification with high-scale thresholds. Phys Rev. (2015) D91:075016. doi: 10.1103/PhysRevD.91.075016

118. Pontecorvo B. Neutrino experiments and the problem of conservation of leptonic charge. Sov Phys JETP. (1968) 26:984-8. doi: 10.1016/B978-0-12-395657-6.50020-3

119. Schechter J, Valle JWF. Neutrino masses in SU(2) x U(1) theories. Phys Rev. (1980) D22:2227. doi: 10.1103/PhysRevD.22.2227

120. Ma E. Pathways to naturally small neutrino masses. Phys Rev Lett. (1998) 81:1171-4. doi: 10.1103/PhysRevLett.81.1171

121. Minkowski P. $\mu \rightarrow e \gamma$ at a rate of one out of $10^{9}$ muon decays? Phys Lett. (1977) 67B:421-8. doi: 10.1016/0370-2693(77)90435-X

122. Yanagida T. HORIZONTAL SYMMETRY AND MASSES OF NEUTRINOS. Conf Proc. (1979) C7902131:95-9.

123. Gell-Mann M, Ramond P, Slansky R. Complex spinors and unified theories. Conf Proc. (1979) C790927:315-21.

124. Magg M, Wetterich C. Neutrino mass problem and gauge hierarchy. Phys Lett. (1980) 94B:61-4. doi: 10.1016/0370-2693(80)90825-4

125. Lazarides G, Shafi Q, Wetterich C. Proton lifetime and fermion masses in an SO(10) model. Nucl Phys. (1981) B181:287-300. doi: 10.1016/0550-3213(81)90354-0

126. Goh HS, Mohapatra RN, Nasri S. SO(10) symmetry breaking and type II seesaw. Phys Rev. (2004) D70:075022. doi: 10.1103/PhysRevD.70.075022

127. Mohapatra RN, Parida MK. Type II seesaw dominance in nonsupersymmetric and split susy $\mathrm{SO}(10)$ and proton life time. Phys Rev. (2011) D84:095021. doi: 10.1103/PhysRevD.84.095021

128. Foot R, Lew H, He XG, Joshi GC. Seesaw neutrino masses induced by a triplet of leptons. Z Phys. (1989) C44:441. doi: 10.1007/BF01415558

129. Fileviez Perez P. Supersymmetric adjoint SU(5). Phys Rev. (2007) D76:071701. doi: 10.1103/PhysRevD.76.071701

130. Gonzalez-Garcia MC, Valle JWF. Fast decaying neutrinos and observable flavor violation in a new class of majoron models. Phys Lett. (1989) B216:360-6. doi: 10.1016/0370-2693(89)91131-3

131. 't Hooft G. Naturalness, chiral symmetry, and spontaneous chiral symmetry breaking. NATO Sci Ser B. (1980) 59:135-57. doi: 10.1007/978-1-4684-7571-5_9

132. Malinsky M, Romao JC, Valle JWF. Novel supersymmetric SO(10) seesaw mechanism. Phys Rev Lett. (2005) 95:161801. doi: 10.1103/PhysRevLett.95.161801

133. Hirsch M, Valle JWF. Supersymmetric origin of neutrino mass. New J Phys. (2004) 6:76. doi: 10.1088/1367-2630/6/1/076

134. Babu KS. Model of 'Calculable' majorana neutrino masses. Phys Lett. (1988) B203:132-6. doi: 10.1016/0370-2693(88)91584-5

135. Witten E. Neutrino masses in the minimal O(10) theory. Phys Lett. (1980) 91B:81-4. doi: 10.1016/0370-2693(80)90666-8

136. Leontaris GK, Vergados JD. The Seesaw mechanism in string models. Phys Lett. (1991) B258:111-7. doi: 10.1016/0370-2693(91)91217-J

137. Arbeláez Rodríguez $C$, Kolešová $H$, Malinský M. Witten's mechanism in the flipped SU(5) unification. Phys Rev D. (2014) 89:055003. doi: 10.1103/PhysRevD.89.055003

138. Harries D, Malinský M, Zdráhal M. Witten's loop in the minimal flipped SU(5) unification revisited. Phys Rev D. (2018) 98:095015. doi: 10.1103/PhysRevD.98.095015

139. Giveon A, Hall LJ, Sarid U. SU(5) unification revisited. Phys Lett. (1991) B271:138-44. doi: 10.1016/0370-2693(91)91289-8

140. Antusch S, King SF, Spinrath M. GUT predictions for quark-lepton Yukawa coupling ratios with messenger masses from non-singlets. Phys Rev D. (2014) 89:055027. doi: 10.1103/PhysRevD.89.055027

141. Ellis JR, Gaillard MK. Fermion masses and higgs representations in SU(5). Phys Lett. (1979) 88B:315-9. doi: 10.1016/0370-2693(79)90476-3
142. Kelley S, Lopez JL, Nanopoulos DV. Yukawa unification. Phys Lett. (1992) B274:387-92. doi: 10.1016/0370-2693(92)92003-Y

143. Ananthanarayan B, Lazarides G, Shafi Q. Top mass prediction from supersymmetric guts. Phys Rev. (1991) D44:1613-5. doi: 10.1103/PhysRevD.44.1613

144. Antusch S, Spinrath M. Quark and lepton masses at the GUT scale including SUSY threshold corrections. Phys Rev. (2008) D78:075020. doi: 10.1103/PhysRevD.78.075020

145. Antusch S, Spinrath M. New GUT predictions for quark and lepton mass ratios confronted with phenomenology. Phys Rev. (2009) D79:095004. doi: 10.1103/PhysRevD.79.095004

146. Babu KS, Kolda CF. Signatures of supersymmetry and Yukawa unification in Higgs decays. Phys Lett. (1999) B451:77-85. doi: 10.1016/S0370-2693(99)00204-X

147. Olechowski M, Pokorski S. Electroweak symmetry breaking with nonuniversal scalar soft terms and large tan beta solutions. Phys Lett. (1995) B344:201-10. doi: 10.1016/0370-2693(94)01571-S

148. Blazek T, Dermisek R, Raby S. Yukawa unification in $\mathrm{SO}(10)$. Phys Rev. (2002) D65:115004. doi: 10.1103/PhysRevD.65. 115004

149. Hall LJ, Rattazzi R, Sarid U. The Top quark mass in supersymmetric SO(10) unification. Phys Rev. (1994) D50:7048-65. doi: 10.1103/PhysRevD.50.7048

150. King SF, Oliveira M. Yukawa unification as a window into the soft supersymmetry breaking Lagrangian. Phys Rev. (2001) D63:015010. doi: 10.1103/PhysRevD.63.015010

151. Baer H, Raza S, Shafi Q. A heavier gluino from t-b-t Yukawa-unified SUSY. Phys Lett. (2012) B712:250-4. doi: 10.1016/j.physletb.2012.04.075

152. Langacker P, Polonsky N. The Bottom mass prediction in supersymmetric grand unification: Uncertainties and constraints. Phys Rev. (1994) D49:1454-67. doi: 10.1103/PhysRevD.49.1454

153. Langacker P, Polonsky N. Implications of Yukawa unification for the Higgs sector in supersymmetric grand unified models. Phys Rev. (1994) D50:2199217. doi: 10.1103/PhysRevD.50.2199

154. Baer H, Gogoladze I, Mustafayev A, Raza S, Shafi Q. Sparticle mass spectra from SU(5) SUSY GUT models with $b-\tau$ Yukawa coupling unification. $J$ High Energy Phys. (2012) 03:047. doi: 10.1007/JHEP03(2012)047

155. Gomez ME, Lazarides G, Pallis C. Yukawa quasi-unification. Nucl Phys. (2002) B638:165-85. doi: 10.1016/S0550-3213(02)00483-2

156. Gogoladze I, Khalid R, Raza S, Shafi Q. $t-b-\tau$ Yukawa unification for $\mu<0$ with a sub-TeV sparticle spectrum. J High Energy Phys. (2010) 12:055. doi: 10.1007/JHEP12(2010)055

157. Bajc B, Senjanovic G, Vissani F. b - tau unification and large atmospheric mixing: a Case for noncanonical seesaw. Phys Rev Lett. (2003) 90:051802. doi: 10.1103/PhysRevLett.90.051802

158. Anderson G, Raby S, Dimopoulos S, Hall LJ, Starkman GD. A Systematic SO(10) operator analysis for fermion masses. Phys Rev. (1994) D49:3660-90. doi: 10.1103/PhysRevD.49.3660

159. Altarelli G, Feruglio F. Discrete flavor symmetries and models of neutrino mixing. Rev Mod Phys. (2010) 82:2701-29. doi: 10.1103/RevModPhys.82.2701

160. Ishimori H, Kobayashi T, Ohki H, Shimizu Y, Okada H, Tanimoto M. Nonabelian discrete symmetries in particle physics. Prog Theor Phys Suppl. (2010) 183:1-163. doi: 10.1143/PTPS.183.1

161. Grimus W, Ludl PO. Finite flavour groups of fermions. J Phys. (2012) A45:233001. doi: 10.1088/1751-8113/45/23/233001

162. King SF, Luhn C, Stuart AJ. A grand Delta(96) x SU(5) flavour model. Nucl Phys. (2013) B867:203-35. doi: 10.1016/j.nuclphysb.2012.09.021

163. de Anda FJ, King SF. SU(3) $\times S O(10)$ in 6d. J High Energy Phys. (2018) 10:128. doi: 10.1007/JHEP10(2018)128

164. Arbeláez C, Hirsch M, Malinský M, Romão JC. LHC-scale leftright symmetry and unification. Phys Rev. (2014) D89:035002. doi: 10.1103/PhysRevD.89.035002

165. Deppisch FF, Gonzalo TE, Graf L. Surveying the SO(10) model landscape: the left-right symmetric case. Phys Rev. (2017) D96:055003. doi: 10.1103/PhysRevD.96.055003

166. Senjanovic G. Seesaw at LHC through left-right symmetry. Int J Mod Phys. (2011) A26:1469-91. doi: 10.1142/S0217751X1105302X 
167. Nemevsek M, Nesti F, Senjanovic G, Zhang Y. First limits on leftright symmetry scale from LHC data. Phys Rev. (2011) D83:115014. doi: 10.1103/PhysRevD.83.115014

168. Han T, Lewis I, Ruiz R, Si Zg. Lepton number violation and $W^{\prime}$ chiral couplings at the LHC. Phys Rev. (2013) D87:035011. doi: 10.1103/PhysRevD.87.039906

169. Das SP, Deppisch FF, Kittel O, Valle JWF. Heavy neutrinos and lepton flavour violation in left-right symmetric models at the LHC. Phys Rev. (2012) D86:055006. doi: 10.1103/PhysRevD.86.055006

170. Bambhaniya G, Chakrabortty J, Gluza J, Kordiaczynska M, Szafron R. Leftright symmetry and the charged higgs bosons at the LHC. J High Energy Phys. (2014) 05:033. doi: 10.1007/JHEP05(2014)033

171. Chen CY, Dev PSB, Mohapatra RN. Probing heavy-light neutrino mixing in left-right seesaw models at the LHC. Phys Rev D. (2013) 88:033014. doi: 10.1103/PhysRevD.88.033014

172. Lee CH, Bhupal Dev PS, Mohapatra RN. Natural TeV-scale left-right seesaw mechanism for neutrinos and experimental tests. Phys Rev D. (2013) 88:093010. doi: 10.1103/PhysRevD.88.093010

173. Deppisch FF, Gonzalo TE, Patra S, Sahu N, Sarkar U. Signal of righthanded charged gauge bosons at the LHC? Phys Rev D. (2014) 90:053014. doi: 10.1103/PhysRevD.90.053014

174. Deppisch FF, Gonzalo TE, Patra S, Sahu N, Sarkar U. Double beta decay, lepton flavor violation, and collider signatures of left-right symmetric models with spontaneous D-parity breaking. Phys Rev D. (2015) 91:015018. doi: 10.1103/PhysRevD.91.015018

175. Deppisch FF, Graf L, Kulkarni S, Patra S, Rodejohann W, Sahu N, et al. Reconciling the $2 \mathrm{TeV}$ excesses at the LHC in a linear seesaw left-right model. Phys Rev D. (2016) 93:013011. doi: 10.1103/PhysRevD.93.013011

176. Bhupal Dev PS, Mohapatra RN. Unified explanation of the eejj, diboson and dijet resonances at the LHC. Phys Rev Lett. (2015) 115:181803. doi: 10.1103/PhysRevLett.115.181803

177. Mitra M, Ruiz R, Scott DJ, Spannowsky M. Neutrino jets from high-mass $W_{R}$ gauge bosons in TeV-Scale left-right symmetric models. Phys Rev D. (2016) 94:095016. doi: 10.1103/PhysRevD.94.095016

178. Helo JC, Li H, Neill NA, Ramsey-Musolf M, Vasquez JC. Probing neutrino Dirac Mass in Left-Right Symmetric Models at the LHC and Next Generation Colliders. Phys Rev D. (2018) 99:055042. doi: 10.1103/PhysRevD.99.055042

179. Mohapatra RN. Mechanism for understanding small neutrino mass in superstring theories. Phys Rev Lett. (1986) 56:561-3. doi: 10.1103/PhysRevLett.56.561

180. Dev PSB, Mohapatra RN. TeV scale inverse seesaw in SO(10) and leptonic non-unitarity effects. Phys Rev. (2010) D81:013001. doi: 10.1103/PhysRevD.81.013001

181. Brdar V, Smirnov AY. Low scale left-right symmetry and naturally small neutrino mass. J High Energy Phys. (2018) 1902:045. doi: 10.1007/JHEP02(2019)045

182. Akhmedov EK, Lindner M, Schnapka E, Valle JWF. Left-right symmetry breaking in NJL approach. Phys Lett. (1996) B368:270-80. doi: 10.1016/0370-2693(95)01504-3

183. Akhmedov EK, Lindner M, Schnapka E, Valle JWF. Dynamical left-right symmetry breaking. Phys Rev. (1996) D53:2752-80. doi: 10.1103/PhysRevD.53.2752

184. Fileviez Perez P. Type III Seesaw and Left-Right Symmetry. J High Energy Phys. (2009) 03:142. doi: 10.1088/1126-6708/2009/03/142

185. Duerr M, Fileviez Perez P, Lindner M. Left-right symmetric theory with light sterile neutrinos. Phys Rev D. (2013) 88:051701. doi: 10.1103/PhysRevD.88.051701

186. Fileviez Perez P, Murgui C, Ohmer S. Simple left-right theory: lepton number violation at the LHC. Phys Rev D. (2016) 94:051701. doi: 10.1103/PhysRevD.94.051701

187. Babu KS, Mohapatra RN. Predictive neutrino spectrum in minimal SO(10) grand unification. Phys Rev Lett. (1993) 70:2845-8. doi: 10.1103/PhysRevLett.70.2845

188. Albright $\mathrm{CH}$, Barr SM. Construction of a minimal Higgs SO(10) SUSY GUT model. Phys Rev. (2000) D62:093008. doi: 10.1103/PhysRevD. 62.093008
189. Fukuyama T, Okada N. Neutrino oscillation data versus minimal supersymmetric SO(10) model. J High Energy Phys. (2002) 11:011. doi: 10.1088/1126-6708/2002/11/011

190. Goh HS, Mohapatra RN, Ng SP. Minimal SUSY SO(10) model and predictions for neutrino mixings and leptonic CP violation. Phys Rev. (2003) D68:115008. doi: 10.1103/PhysRevD.68.115008

191. Goh HS, Mohapatra RN, Ng SP. Minimal SUSY SO(10), b tau unification and large neutrino mixings. Phys Lett. (2003) B570:215-21. doi: 10.1016/j.physletb.2003.08.011

192. Bertolini S, Frigerio M, Malinsky M. Fermion masses in SUSY SO(10) with type II seesaw: a Non-minimal predictive scenario. Phys Rev. (2004) D70:095002. doi: 10.1103/PhysRevD.70.095002

193. Matsuda K, Koide Y, Fukuyama T. Can the SO(10) model with two Higgs doublets reproduce the observed fermion masses? Phys Rev. (2001) D64:053015. doi: 10.1103/PhysRevD.64.053015

194. Matsuda K, Koide Y, Fukuyama T, Nishiura H. How far can the SO(10) two Higgs model describe the observed neutrino masses and mixings? Phys Rev. (2002) D65:033008. doi: 10.1103/PhysRevD.65.033008

195. Babu KS, Macesanu C. Neutrino masses and mixings in a minimal SO(10) model. Phys Rev. (2005) D72:115003. doi: 10.1103/PhysRevD.72.115003

196. Dueck A, Rodejohann W. Fits to SO(10) Grand Unified Models. J High Energy Phys. (2013) 09:024. doi: 10.1007/JHEP09(2013)024

197. Deppisch T, Schacht S, Spinrath M. Confronting SUSY SO(10) with updated lattice and neutrino data. J High Energy Phys. (2019) 01:005. doi: 10.1007/JHEP01(2019)005

198. Aulakh CS, Bajc B, Melfo A, Senjanovic G, Vissani F. The Minimal supersymmetric grand unified theory. Phys Lett. (2004) B588:196-202. doi: 10.1016/j.physletb.2004.03.031

199. Aulakh CS, Girdhar A. SO(10) MSGUT: Spectra, couplings and threshold effects. Nucl Phys. (2005) B711:275-313. doi: 10.1016/j.nuclphysb.2005.01.008

200. Bajc B, Melfo A, Senjanovic G, Vissani F. The Minimal supersymmetric grand unified theory. 1. Symmetry breaking and the particle spectrum. Phys Rev. (2004) D70:035007. doi: 10.1103/PhysRevD.70.035007

201. Bajc B, Melfo A, Senjanovic G, Vissani F. Fermion mass relations in a supersymmetric $\mathrm{SO}(10)$ theory. Phys Lett. (2006) B634:272-7. doi: 10.1063/1.2149692

202. Bajc B, Dorsner I, Nemevsek M. Minimal SO(10) splits supersymmetry. J High Energy Phys. (2008) 11:007. doi: 10.1088/1126-6708/2008/11/007

203. De Romeri V, Hirsch M, Malinsky M. Soft masses in SUSY SO(10) GUTs with low intermediate scales. Phys Rev. (2011) D84:053012. doi: 10.1103/PhysRevD.84.053012

204. Deppisch FF, Desai N, Gonzalo TE. Compressed and Split Spectra in Minimal SUSY SO(10). Frontin Phys. (2014) 2:27. doi: 10.3389/fphy.2014.00027

205. Poh Z, Raby S. Yukawa Unification in an SO(10) SUSY GUT: SUSY on the Edge. Phys Rev D. (2015) 92:015017. doi: 10.1103/PhysRevD.92.015017

206. Ellis SAR, Gherghetta T, Kaneta K, Olive KA. New Weak-Scale Physics from SO(10) with High-Scale Supersymmetry. Phys Rev D. (2018) 98:055009. doi: 10.1103/PhysRevD.98.055009

207. Dutta B, Mimura Y, Mohapatra RN. Suppressing proton decay in the minimal SO(10) model. Phys Rev Lett. (2005) 94:091804. doi: 10.1103/PhysRevLett.94.091804

208. Aulakh CS, Garg SK. The new minimal supersymmetric GUT: spectra, RG analysis and fermion fits. Nucl Phys. (2012) B857:101-42. doi: 10.1016/j.nuclphysb.2011.12.003

209. Fukuyama T, Ilakovac A, Kikuchi T, Meljanac S, Okada N. SO(10) group theory for the unified model building. J Math Phys. (2005) 46:033505. doi: 10.1063/1.1847709

210. Babu KS, Bajc B, Saad S. Resurrecting minimal yukawa sector of SUSY SO(10). J High Energy Phys. (2018) 10:135. doi: 10.1007/JHEP10(2018)135

211. Bhupal Dev PS, Mohapatra RN. Electroweak symmetry breaking and proton decay in SO(10) SUSY-GUT with TeV W(R). Phys Rev D. (2010) 82:035014. doi: 10.1103/PhysRevD.82.035014

212. Anandakrishnan A, Bryant BC, Raby S, Wingerter A. LHC phenomenology of SO(10) models with Yukawa Unification. Phys Rev D. (2013) 88:075002. doi: 10.1103/PhysRevD.88.075002 
213. Baer H, Kraml S, Sekmen S, Summy H. Dark matter allowed scenarios for Yukawa-unified SO(10) SUSY GUTs. J High Energy Phys. (2008) 03:056. doi: 10.1088/1126-6708/2008/03/056

214. Goh HS, Mohapatra RN, Nasri S, Ng SP. Proton decay in a minimal SUSY SO(10) model for neutrino mixings. Phys Lett. (2004) B587:105-16. doi: 10.1016/j.physletb.2004.02.063

215. Mohapatra RN, Severson M. Leptonic CP violation and proton decay in SUSY SO(10). J High Energy Phys. (2018) 09:119. doi: 10.1007/JHEP09(2018)119

216. Masiero A, Vempati SK, Vives O. Seesaw and lepton flavor violation in SUSY SO(10). Nucl Phys. (2003) B649:189-204. doi: 10.1016/S0550-3213(02)01031-3

217. Dermisek R, Raby S, Roszkowski L, Ruiz de Austri R. Dark matter and $\mathrm{B}(\mathrm{s}) \rightarrow \mathrm{mu}+\mathrm{mu}-$ with minimal $\mathrm{SO}(10)$ soft SUSY breaking II. J High Energy Phys. (2005) 09:029. doi: 10.1088/1126-6708/2005/ 09/029

218. Babu KS, He XG, Pakvasa S. Neutrino masses and proton decay modes in $\mathrm{SU}(3) \mathrm{X} \mathrm{SU}(3) \mathrm{X} \mathrm{SU}(3)$ trinification. Phys Rev. (1986) D33:763. doi: 10.1103/PhysRevD.33.763

219. Sayre J, Wiesenfeldt S, Willenbrock S. Minimal trinification. Phys Rev. (2006) D73:035013. doi: 10.1103/PhysRevD.73.035013

220. Stech B. The mass of the Higgs boson in the trinification subgroup of E6. Phys Rev. (2012) D86:055003. doi: 10.1103/PhysRevD.86.055003

221. Willenbrock S. Triplicated trinification. Phys Lett. (2003) B561:130-4. doi: 10.1016/S0370-2693(03)00419-2

222. Wang MY, Carlson ED. The Breaking of the $\mathrm{SU}(3) * * 3$ gauge group. arXiv:hep-ph/9302215 (1992).

223. Cauet C, Pas H, Wiesenfeldt S. Trinification, the hierarchy problem and inverse seesaw neutrino masses. Phys Rev. (2011) D83:093008. doi: 10.1103/PhysRevD.83.093008

224. Dvali GR, Shafi Q. Gauge hierarchy in $\mathrm{SU}(3)(\mathrm{C}) \times \mathrm{SU}(3)(\mathrm{L}) \times \mathrm{SU}(3)-$ $\mathrm{R}$ and low-energy implications. Phys Lett. (1994) B326:258-63. doi: 10.1016/0370-2693(94)91319-6

225. Carone CD. Tri-N-ification. Phys Rev. (2005) D71:075013. doi: 10.1103/PhysRevD.71.075013

226. Nath P, Arnowitt RL. Symmetry breaking in three generation calabi-yau manifolds. Phys Rev. (1989) D39:2006.

227. Maekawa N, Shafi Q. Supersymmetric $\mathrm{SU}^{(3)} 3$ unification with anomalous U(1)(a) gauge symmetry. Prog Theor Phys. (2003) 109:279-93. doi: 10.1143/PTP.109.279

228. Hetzel J, Stech B. Low-energy phenomenology of trinification: an effective left-right-symmetric model. Phys Rev. (2015) D91:055026. doi: 10.1103/PhysRevD.91.055026

229. Stech B. Trinification Phenomenology and the structure of Higgs Bosons. J High Energy Phys. (2014) 08:139. doi: 10.1007/JHEP08(2014)139

230. Camargo-Molina JE, Morais AP, Pasechnik R, Wessén J. On a radiative origin of the Standard Model from Trinification. J High Energy Phys. (2016) 09:129. doi: 10.1007/JHEP09(2016)129

231. Camargo-Molina JE, Morais AP, Ordell A, Pasechnik R, Sampaio MOP, Wessén J. Reviving trinification models through an E6 extended supersymmetric GUT. Phys Rev. (2017) D95:075031. doi: 10.1103/PhysRevD.95.075031

232. Camargo-Molina JE, Morais AP, Ordell A, Pasechnik R, Wessén J. Scale hierarchies, symmetry breaking and particle spectra in SU(3)family extended SUSY trinification. Phys Rev. (2019) D99:035041. doi: 10.1103/PhysRevD.99.035041

233. King SF, Moretti S, Nevzorov R. Theory and phenomenology of an exceptional supersymmetric standard model. Phys Rev. (2006) D73:035009. doi: 10.1103/PhysRevD.73.035009

234. King SF, Moretti S, Nevzorov R. Exceptional supersymmetric standard model. Phys Lett. (2006) B634:278-84. doi: 10.1016/j.physletb.2005. 12.070

235. Athron P, Hall JP, Howl R, King SF, Miller DJ, Moretti S, et al. Aspects of the exceptional supersymmetric standard model. Nucl Phys Proc Suppl. (2010) 200-202:120-9. doi: 10.1016/j.nuclphysbps.2010.02.074

236. King SF, Luo R, Miller DJ, Nevzorov R. Leptogenesis in the exceptional supersymmetric standard model: flavour dependent lepton asymmetries.
J High Energy Phys. (2008) 12:042. doi: 10.1088/1126-6708/2008/ $12 / 042$

237. Nevzorov R. Leptogenesis as an origin of hot dark matter and baryon asymmetry in the $E_{6}$ inspired SUSY models. Phys Lett. (2018) B779:223-9. doi: $10.1016 /$ j.physletb.2018.02.020

238. Nevzorov R. $E_{6}$ inspired supersymmetric models with exact custodial symmetry. Phys Rev. (2013) D87:015029. doi: 10.1103/PhysRevD.87.015029

239. Nevzorov R. Quasifixed point scenarios and the Higgs mass in the E6 inspired supersymmetric models. Phys Rev. (2014) D89:055010. doi: 10.1103/PhysRevD.89.055010

240. Howl R, King SF. Planck scale unification in a supersymmetric standard model. Phys Lett. (2007) B652:331-7. doi: 10.1016/j.physletb.2007.07.035

241. Athron P, King SF, Miller DJ, Moretti S, Nevzorov R. The constrained exceptional supersymmetric standard model. Phys Rev. (2009) D80:035009. doi: 10.1103/PhysRevD.80.035009

242. Athron P, King SF, Miller DJ, Moretti S, Nevzorov R. Predictions of the constrained exceptional supersymmetric standard model. Phys Lett. (2009) B681:448-56. doi: 10.1016/j.physletb.2009.10.051

243. Athron P, King SF, Miller DJ, Moretti S, Nevzorov R. Constrained Exceptional Supersymmetric Standard Model with a Higgs Near 125 GeV. Phys Rev. (2012) D86:095003. doi: 10.1103/PhysRevD.86. 095003

244. Athron P, Mühlleitner M, Nevzorov R, Williams AG. Non-Standard Higgs Decays in U(1) Extensions of the MSSM. J High Energy Phys. (2015) 01:153. doi: 10.1007/JHEP01(2015)153

245. Athron P, King SF, Miller DJ, Moretti S, Nevzorov R. LHC signatures of the constrained exceptional supersymmetric standard model. Phys Rev. (2011) D84:055006. doi: 10.1103/PhysRevD. 84.055006

246. Hall JP, King SF. Neutralino dark matter with inert higgsinos and singlinos. J High Energy Phys. (2009) 08:088. doi: 10.1088/1126-6708/2009/08/088

247. Hall JP, King SF. Bino dark matter and big bang nucleosynthesis in the constrained $E_{6}$ SSM with massless inert singlinos. J High Energy Phys. (2011) 06:006. doi: 10.1007/JHEP06(2011)006

248. Hall JP, King SF, Nevzorov R, Pakvasa S, Sher M, Nevzorov R, et al. Novel Higgs Decays and Dark Matter in the E(6)SSM. Phys Rev. (2011) D83:075013. doi: 10.1103/PhysRevD.83.075013

249. Athron P, Harries D, Nevzorov R, Williams AG. E6 Inspired SUSY benchmarks, dark matter relic density and a $125 \mathrm{GeV}$ Higgs. Phys Lett. (2016) B760:19-25. doi: 10.1016/j.physletb.2016.06.040

250. Athron P, Harries D, Nevzorov R, Williams AG. Dark matter in a constrained $\mathrm{E}_{6}$ inspired SUSY model. J High Energy Phys. (2016) 12:128. doi: 10.1007/JHEP12(2016)128

251. Rocher J, Sakellariadou M. Supersymmetric grand unified theories and cosmology. J Cosmol Astropart Phys. (2005) 0503:004. doi: 10.1088/1475-7516/2005/03/004

252. Lyth $\mathrm{DH}$. What would we learn by detecting a gravitational wave signal in the cosmic microwave background anisotropy? Phys Rev Lett. (1997) 78:1861-3. doi: 10.1103/PhysRevLett.78.1861

253. Ade PAR, Aghanim N, Arnaud M, Arroja F, Ashdown M, Aumont J, et al. Planck 2015 results. XX. Constraints on inflation. Astron Astrophys. (2016) 594:A20. doi: 10.1051/0004-6361/201525898

254. Akrami Y, Arroja F, Ashdown M, Aumont J, Baccigalupi C, Ballardini M, et al. Planck 2018 results. X. Constraints on inflation. arXiv:1807.06211 (2018).

255. Coleman SR, Weinberg EJ. Radiative corrections as the origin of spontaneous symmetry breaking. Phys Rev. (1973) D7:1888-910. doi: 10.1103/PhysRevD.7.1888

256. Croon D, Sanz V. Saving natural inflation. J Cosmol Astropart Phys. (2015) 1502:008. doi: 10.1088/1475-7516/2015/02/008

257. Croon D, Sanz V, Setford J. Goldstone inflation. J High Energy Phys. (2015) 10:020. doi: 10.1007/JHEP10(2015)020

258. Croon D, Sanz V, Tarrant ERM. Reheating with a composite Higgs boson. Phys Rev. (2016) D94:045010. doi: 10.1103/PhysRevD.94.045010

259. Shafi Q, Vilenkin A. Inflation with SU(5). Phys Rev Lett. (1984) 52:691-4. doi: 10.1103/PhysRevLett.52.691

260. Barenboim G, Chun EJ, Lee HM. Coleman-Weinberg Inflation in light of Planck. Phys Lett. (2014) B730:81-88. doi: 10.1016/j.physletb.2014.01.039 
261. Okada N, Senoguz VN, Shafi Q. The observational status of simple inflationary models: an update. Turk J Phys. (2016) 40:150-62. doi: $10.3906 /$ fiz-1505-7

262. Cerioni A, Finelli F, Tronconi A, Venturi G. Inflation and reheating in induced gravity. Phys Lett. (2009) B681:383-6. doi: 10.1016/j.physletb.2009.10.066

263. Panotopoulos G. Nonminimal GUT inflation after Planck results. Phys Rev. (2014) D89:047301. doi: 10.1103/PhysRevD.89.047301

264. Karam A, Pappas T, Tamvakis K. Nonminimal coleman-weinberg inflation with an $R^{2}$ term. J Cosmol Astropar Phys. (2018) 2019:006. doi: 10.1088/1475-7516/2019/02/006

265. Ellis J, Nanopoulos DV, Olive KA. No-Scale supergravity realization of the starobinsky model of inflation. Phys Rev Lett. (2013) 111:111301. doi: 10.1103/PhysRevLett.111.129902

266. Starobinsky AA. A new type of isotropic cosmological models without singularity. Phys Lett. (1980) B91:99-102. doi: 10.1016/0370-2693(80)90670-X

267. Whitt B. Fourth order gravity as general relativity plus matter. Phys Lett. (1984) 145B:176-8. doi: 10.1016/0370-2693(84)90332-0

268. Croon D, Ellis J, Mavromatos NE. Wess-zumino inflation in light of planck. Phys Lett. (2013) B724:165-9. doi: 10.1016/j.physletb.2013.06.016

269. Ellis J, Nanopoulos DV, Olive KA. A no-scale supergravity framework for sub-Planckian physics. Phys Rev. (2014) D89:043502. doi: 10.1103/PhysRevD.89.043502

270. Ellis J, Gonzalo TE, Harz J, Huang WC. Flipped GUT Inflation. J Cosmol Astropart Phys. (2015) 1503:039. doi: 10.1088/1475-7516/2015/03/039

271. Ellis J, Garcia MAG, Nagata N, Nanopoulos DV, Olive KA. Starobinskylike inflation and neutrino masses in a No-Scale $\mathrm{SO}(10)$ model. J Cosmol Astropart Phys. (2016) 1611:018. doi: 10.1088/1475-7516/2016/ $11 / 018$

272. Gonzalo TE, Heurtier L, Moursy A. Sneutrino driven GUT inflation in supergravity. J High Energy Phys. (2017) 06:109. doi: 10.1007/JHEP06(2017)109

273. Ellis J, He HJ, Xianyu ZZ. New higgs inflation in a No-Scale supersymmetric SU(5) GUT. Phys Rev. (2015) D91:021302. doi: 10.1103/PhysRevD.91.021302

274. Ellis J, He HJ, Xianyu ZZ. Higgs inflation, reheating and gravitino production in No-Scale supersymmetric GUTs. J Cosmol Astropart Phys. (2016) 1608:068. doi: 10.1088/1475-7516/2016/08/068

275. Hertzberg MP, Wilczek F. Inflation driven by unification energy. Phys Rev. (2017) D95:063516. doi: 10.1103/PhysRevD.95.063516

276. Rehman MU, Shafi Q, Wickman JR. GUT inflation and proton decay after WMAP5. Phys Rev. (2008) D78:123516. doi: 10.1103/PhysRevD.78.123516

277. Boucenna SM, Morisi S, Shafi Q, Valle JWF. Inflation and majoron dark matter in the seesaw mechanism. Phys Rev. (2014) D90:055023. doi: 10.1103/PhysRevD.90.055023

278. Sravan Kumar K, Vargas Moniz P. Conformal GUT inflation, proton lifetime and non-thermal leptogenesis. arXiv:1806.09032 (2018).

279. Kibble TWB. Topology of cosmic domains and strings. J Phys. (1976) A9:1387-98. doi: 10.1088/0305-4470/9/8/029

280. Kibble TWB. Some implications of a cosmological phase transition. Phys Rept. (1980) 67:183. doi: 10.1016/0370-1573(80)90091-5

281. Jeannerot R, Rocher J, Sakellariadou M. How generic is cosmic string formation in SUSY GUTs. Phys Rev. (2003) D68:103514. doi: 10.1103/PhysRevD.68.103514

282. Achucarro A, Martins CJAP. Cosmic strings. In: Meyers, RA editor. Encyclopedia of Complexity and Systems Science. New York, NY: Springer (2009). doi: 10.1007/978-0-387-30440-3_107

283. Vilenkin A. Cosmological density fluctuations produced by vacuum strings. Phys Rev Lett. (1981) 46:1169-72. doi: 10.1103/PhysRevLett.46.1169

284. Brandenberger RH, Turok N. Fluctuations from cosmic strings and the microwave background. Phys Rev. (1986) D33:2182. doi: 10.1103/PhysRevD.33.2182

285. Gott JR III. Gravitational lensing effects of vacuum strings: exact solutions. Astrophys J. (1985) 288:422-7. doi: 10.1086/162808

286. Kaiser N, Stebbins A. Microwave anisotropy due to cosmic strings. Nature. (1984) 310:391-3. doi: 10.1038/310391a0
287. Ade PAR, Aghanim N, Arnaud M, Ashdown M, Aumont J, Baccigalupi C, et al. Planck 2015 results. XIII. Cosmological parameters. Astron Astrophys. (2016) 594:A13. doi: 10.1051/0004-6361/201525830

288. Lizarraga J, Urrestilla J, Daverio D, Hindmarsh M, Kunz M. New CMB constraints for Abelian Higgs cosmic strings. J Cosmol Astropart Phys. (2016) 1610:042. doi: 10.1088/1475-7516/2016/10/042

289. Vilenkin A. Gravitational radiation from cosmic strings. Phys Lett. (1981) 107B:47-50. doi: 10.1016/0370-2693(81)91144-8

290. Vachaspati T, Vilenkin A. Gravitational radiation from cosmic strings. Phys Rev. (1985) D31:3052. doi: 10.1103/PhysRevD.31.3052

291. Caldwell RR, Allen B. Cosmological constraints on cosmic string gravitational radiation. Phys Rev. (1992) D45:3447-68. doi: 10.1103/PhysRevD.45.3447

292. Siemens X, Mandic V, Creighton J. Gravitational wave stochastic background from cosmic (super)strings. Phys Rev Lett. (2007) 98:111101. doi: 10.1103/PhysRevLett.98.111101

293. Sanidas SA, Battye RA, Stappers BW. Projected constraints on the cosmic (super)string tension with future gravitational wave detection experiments. Astrophys J. (2013) 764:108. doi: 10.1088/0004-637X/764/ $1 / 108$

294. Damour T, Vilenkin A. Gravitational wave bursts from cosmic strings. Phys Rev Lett. (2000) 85:3761-4. doi: 10.1103/PhysRevLett.85.3761

295. Damour T, Vilenkin A. Gravitational wave bursts from cusps and kinks on cosmic strings. Phys Rev. (2001) D64:064008. doi: 10.1103/PhysRevD.64.064008

296. Damour T, Vilenkin A. Gravitational radiation from cosmic (super)strings: bursts, stochastic background, and observational windows. Phys Rev. (2005) D71:063510. doi: 10.1103/PhysRevD.71.063510

297. Siemens X, Creighton J, Maor I, Ray Majumder S, Cannon K, Read J. Gravitational wave bursts from cosmic (super)strings: quantitative analysis and constraints. Phys Rev. (2006) D73:105001. doi: 10.1103/PhysRevD.73.105001

298. Olum KD, Blanco-Pillado JJ. Field theory simulation of Abelian Higgs cosmic string cusps. Phys Rev. (1999) D60:023503. doi: 10.1103/PhysRevD.60.023503

299. Aasi J, Abadie J, Abbott BP, Abbott R, Abbott T, Abernathy $\mathrm{MR}$, et al. Constraints on cosmic strings from the LIGO-Virgo gravitational-wave detectors. Phys Rev Lett. (2014) 112:131101. doi: 10.1103/PhysRevLett.112.131101

300. Sanidas SA, Battye RA, Stappers BW. Constraints on cosmic string tension imposed by the limit on the stochastic gravitational wave background from the European Pulsar Timing Array. Phys Rev. (2012) D85:122003. doi: 10.1103/PhysRevD.85.122003

301. Abbott BP, Abbott R, Abbott TD, Acernese F, Ackley K, Adams $\mathrm{C}$, et al. Constraints on cosmic strings using data from the first Advanced LIGO observing run. Phys Rev. (2018) D97:102002. doi: $10.1103 /$ PhysRevD.97.102002

302. Blanco-Pillado JJ, Olum KD, Siemens X. New limits on cosmic strings from gravitational wave observation. Phys Lett. (2018) B778:392-6. doi: 10.1016/j.physletb.2018.01.050

303. Lorenz L, Ringeval C, Sakellariadou M. Cosmic string loop distribution on all length scales and at any redshift. J Cosmol Astropart Phys. (2010) 1010:003. doi: 10.1088/1475-7516/2010/10/003

304. Ringeval C, Sakellariadou M, Bouchet F. Cosmological evolution of cosmic string loops. J Cosmol Astropart Phys. (2007) 0702:023. doi: 10.1088/1475-7516/2007/02/023

305. Ringeval C, Suyama T. Stochastic gravitational waves from cosmic string loops in scaling. J Cosmol Astropart Phys. (2017) 1712:027. doi: 10.1088/1475-7516/2017/12/027

306. Blanco-Pillado JJ, Olum KD, Shlaer B. The number of cosmic string loops. Phys Rev. (2014) D89:023512. doi: 10.1103/PhysRevD.89.023512

307. Blanco-Pillado JJ, Olum KD. Stochastic gravitational wave background from smoothed cosmic string loops. Phys Rev. (2017) D96:104046. doi: 10.1103/PhysRevD.96.104046

308. Martins CJAP, Shellard EPS. Fractal properties and small-scale structure of cosmic string networks. Phys Rev. (2006) D73:043515. doi: 10.1103/PhysRevD.73.043515 
309. Hindmarsh M, Stuckey S, Bevis N. Abelian higgs cosmic strings: small scale structure and loops. Phys Rev. (2009) D79:123504. doi: 10.1103/PhysRevD.79.123504

310. Hindmarsh M, Rummukainen K, Tenkanen TVI, Weir DJ. Improving cosmic string network simulations. Phys Rev. (2014) D90:043539. doi: 10.1103/PhysRevD.90.043539

311. Hindmarsh M, Lizarraga J, Urrestilla J, Daverio D, Kunz M. Scaling from gauge and scalar radiation in Abelian Higgs string networks. Phys Rev. (2017) D96:023525. doi: 10.1103/PhysRevD.96.023525

312. Brandenberger RH. On the decay of cosmic string loops. Nucl Phys. (1987) B293:812-28. doi: 10.1016/0550-3213(87)90092-7

313. Hindmarsh M, Rummukainen K, Weir DJ. Numerical simulations of necklaces in SU(2) gauge-Higgs field theory. Phys Rev. (2017) D95:063520. doi: 10.1103/PhysRevD.95.063520

314. Hindmarsh M, Rummukainen K, Weir DJ. New solutions for non-Abelian cosmic strings. Phys Rev Lett. (2016) 117:251601. doi: 10.1103/PhysRevLett.117.251601

315. Lopez-Eiguren A, Urrestilla J, Achúcarro A, Avgoustidis A, Martins CJAP. Evolution of Semilocal String Networks: II. Velocity estimators. Phys Rev. (2017) D96:023526. doi: 10.1103/PhysRevD.96.023526

316. Figueroa DG, Hindmarsh M, Urrestilla J. Exact scale-invariant background of gravitational waves from cosmic defects. Phys Rev Lett. (2013) 110:101302. doi: 10.1103/PhysRevLett.110.101302

317. Patel HH, Ramsey-Musolf MJ, Wise MB. Color Breaking in the early universe. Phys Rev. (2013) D88:015003. doi: 10.1103/PhysRevD.88. 015003

318. Ramsey-Musolf MJ, Winslow P, White G. Color breaking baryogenesis. Phys Rev. (2018) D97:123509. doi: 10.1103/PhysRevD.97.123509

319. Long AJ, Tesi A, Wang LT. Baryogenesis at a lepton-number-breaking phase transition. J High Energy Phys. (2017) 10:095. doi: 10.1007/JHEP10(2017)095

320. Schmitz K. The B-L Phase Transition: Implications for Cosmology and Neutrinos. Hamburg U. (2012). Available online at: http://wwwlibrary.desy.de/cgi-bin/showprep.pl?thesis12-039

321. Buchmuller W, Domcke V, Schmitz K. Spontaneous B-L breaking as the origin of the hot early universe. Nucl Phys. (2012) B862:587-632. doi: 10.1016/j.nuclphysb.2012.05.001

322. Buchmüller W, Domcke V, Kamada K, Schmitz K. The gravitational wave spectrum from cosmological $B-L$ breaking. J Cosmol Astropart Phys. (2013) 1310:003. doi: 10.1088/1475-7516/2013/10/003

323. Croon D, Sanz V, White G. Model discrimination in gravitational wave spectra from dark phase transitions. J High Energy Phys. (2018) 08:203. doi: 10.1007/JHEP08(2018)203

324. Schwaller P. Gravitational waves from a dark phase transition. Phys Rev Lett. (2015) 115:181101. doi: 10.1103/PhysRevLett.115.181101

325. Breitbach M, Kopp J, Madge E, Opferkuch T, Schwaller P. Dark, cold, and noisy: constraining secluded hidden sectors with gravitational waves. arXiv:1811.11175 (2018).

326. Baldes I, Garcia-Cely C. Strong gravitational radiation from a simple dark matter model. arXiv:1809.01198 (2018).

327. Croon D, Gonzalo TE, White G. Gravitational waves from a pati-salam phase transition. J High Energy Phys. (2019) 02:083. doi: 10.1007/JHEP02(2019)083

328. Mazumdar A, White G. Cosmic phase transitions: their applications and experimental signatures. J High Energy Phys. (2018) doi: 10.1088/1361-6633/ab1f55. [Epub ahead of print].

329. Caprini C, Figueroa DG. Cosmological backgrounds of gravitational waves. Class Quant Grav. (2018) 35:163001. doi: 10.1088/1361-6382/aac608

330. Weir DJ. Gravitational waves from a first order electroweak phase transition: a brief review. Phil Trans Roy Soc Lond. (2018) A376:20170126. doi: $10.1098 /$ rsta.2017.0126

331. Chao W. Electroweak baryogenesis in the exceptional supersymmetric standard model. J Cosmol Astropart Phys. (2015) 1508:055. doi: 10.1088/1475-7516/2015/08/055

332. Chiang CW, Ramsey-Musolf MJ, Senaha E. Standard model with a complex scalar singlet: cosmological implications and theoretical considerations. Phys Rev. (2018) D97:015005. doi: 10.1103/PhysRevD.97. 015005

333. Profumo S, Ramsey-Musolf MJ, Wainwright CL, Winslow P. Singletcatalyzed electroweak phase transitions and precision Higgs boson studies. Phys Rev. (2015) D91:035018. doi: 10.1103/PhysRevD.91 035018

334. Caprini C, Hindmarsh M, Huber S, Konstandin T, Kozaczuk J, Nardini G, et al. Science with the space-based interferometer eLISA. II: gravitational waves from cosmological phase transitions. J Cosmol Astropart Phys. (2016) 1604:001. doi: 10.1088/1475-7516/2016/04/001

335. Bodeker D, Moore GD. Electroweak bubble wall speed limit. J Cosmol Astropart Phys. (2017) 1705:025. doi: 10.1088/1475-7516/2017/ $05 / 025$

336. Hindmarsh M, Huber SJ, Rummukainen K, Weir DJ. Shape of the acoustic gravitational wave power spectrum from a first order phase transition. Phys Rev. (2017) D96:103520. doi: 10.1103/PhysRevD.96.103520

337. Caprini C, Durrer R, Servant G. The stochastic gravitational wave background from turbulence and magnetic fields generated by a firstorder phase transition. J Cosmol Astropart Phys. (2009) 0912:024. doi: 10.1088/1475-7516/2009/12/024

338. Punturo M, Abernathy M, Acernese F, Allen B, Andersson N, Arun K, et al. The einstein telescope: a third-generation gravitational wave observatory. Class Quant Grav. (2010) 27:194002. doi: 10.1088/0264-9381/27/19/194002

339. Akutsu T, Ando M, Arai K, Arai Y, Araki S, Araya A, et al. KAGRA: 2.5 Generation interferometric gravitational wave detector. Nat Astron. (2018) 3:35-40. doi: 10.1038/s41550-018-0658-y

340. Abbott BP, Abbott R, Abbott TD, Abernathy MR, Ackley K, Adams C, et al. Exploring the Sensitivity of Next Generation Gravitational Wave Detectors. Class Quant Grav. (2017) 34:044001. doi: 10.1088/1361-6382/aa51f4

341. Thrane E, Romano JD. Sensitivity curves for searches for gravitational-wave backgrounds. Phys Rev. (2013) D88:124032. doi: 10.1103/PhysRevD.88.124032

342. Espinosa JR, Konstandin T, No JM, Servant G. Energy budget of cosmological first-order phase transitions. J Cosmol Astropart Phys. (2010) 1006:028. doi: 10.1088/1475-7516/2010/06/028

343. Grojean C, Servant G, Wells JD. First-order electroweak phase transition in the standard model with a low cutoff. Phys Rev. (2005) D71:036001. doi: 10.1103/PhysRevD.71.036001

344. Ellis J, Lewicki M, No JM. On the maximal strength of a firstorder electroweak phase transition and its gravitational wave signal. J Cosmol Astropart Phys. (2018) 04:003. doi: 10.1088/1475-7516/2019/ 04/003

345. White GA. A Pedagogical Introduction to Electroweak Baryogenesis. IOP Concise Physics. Morgan \& Claypool (2016). doi: 10.1088/978-1-6817-4457-5ch1

346. Morrissey DE, Ramsey-Musolf MJ. Electroweak baryogenesis. New J Phys. (2012) 14:125003. doi: 10.1088/1367-2630/14/12/125003

347. Aghanim N, Akrami Y, Ashdown M, Aumont J, Baccigalupi C, Ballardini M, et al. Planck 2018 results. VI. Cosmological parameters. (2018) .

348. Riemer-Sørensen S, Jenssen ES. Nucleosynthesis predictions and high-precision deuterium measurements. Universe. (2017) 3:44. doi: 10.3390/universe3020044

349. Sakharov AD. Violation of CP Invariance, C asymmetry, and baryon asymmetry of the universe. Pisma Zh Eksp Teor Fiz. (1967) 5:32-5.

350. Riotto A. Theories of baryogenesis. In: Proceedings, Summer School in Highenergy physics and cosmology: Trieste, Italy, June 29-July 17, 1998 Trieste (1998). p. 326-436.

351. Gu PH, Sarkar U. SO(10) GUT baryogenesis. Phys Lett. (2008) B663:80-2. doi: 10.1016/j.physletb.2008.04.001

352. Gehrlein J, Petcov ST, Spinrath M, Zhang X. Leptogenesis in an SU(5) $\times \mathrm{A}_{5}$ golden ratio flavour model. Nucl Phys. (2015) B896:311-29. doi: 10.1016/j.nuclphysb.2015.08.019

353. Falcone D, Tramontano F. Leptogenesis with $\mathrm{SU}(5)$ inspired mass matrices. Phys Lett. (2001) B506:1-6. doi: 10.1016/S0370-2693(01)00403-8

354. Pilaftsis A, Underwood TEJ. Resonant leptogenesis. Nucl Phys. (2004) B692:303-45. doi: 10.1016/j.nuclphysb.2004.05.029

355. Blanchet S, Fileviez Perez P. Baryogenesis via leptogenesis in adjoint SU(5). J Cosmol Astropart Phys. (2008) 0808:037. doi: 10.1088/1475-7516/2008/08/037

356. Chianese M, Di Bari P. Strong thermal SO(10)-inspired leptogenesis in the light of recent results from long-baseline neutrino experiments. J High Energy Phys. (2018) 05:073. doi: 10.1007/JHEP05(2018)073 
357. Di Bari P, Re Fiorentin M. A full analytic solution of $S O(10)$ inspired leptogenesis. J High Energy Phys. (2017) 10:029. doi: 10.1007/JHEP10(2017)029

358. Di Bari P, Re Fiorentin M. Supersymmetric $S O(10)$-inspired leptogenesis and a new $\mathrm{N}_{2}$-dominated scenario. J Cosmol Astropart Phys. (2016) 1603:039. doi: 10.1088/1475-7516/2016/03/039

359. Di Bari P, Marzola L, Re Fiorentin M. Decrypting SO(10)inspired leptogenesis. Nucl Phys. (2015) B893:122-57. doi: 10.1016/j.nuclphysb.2015.02.005

360. Di Bari P, Marzola L. SO(10)-inspired solution to the problem of the initial conditions in leptogenesis. Nucl Phys. (2013) B877:719-51. doi: 10.1016/j.nuclphysb.2013.10.027

361. Esteban I, Gonzalez-Garcia MC, Hernandez-Cabezudo A, Maltoni M, Schwetz T. Global analysis of three-flavour neutrino oscillations: synergies and tensions in the determination of $\theta_{2} 3, \delta_{C} P$, and the mass ordering. J High Energy Phys. (2018) 01:106. doi: 10.1007/JHEP01(2019)106

362. Björkeroth F, de Anda FJ, de Medeiros Varzielas I, King SF. Leptogenesis in a $\Delta(27) \times S O(10)$ SUSY GUT. J High Energy Phys. (2017) 01:077. doi: 10.1007/JHEP01(2017)077

363. Björkeroth F, de Anda FJ, de Medeiros Varzielas I, King SF. Towards a complete $\mathrm{A}_{4} \times \mathrm{SU}(5)$ SUSY GUT. J High Energy Phys. (2015) 06:141. doi: 10.1007/JHEP06(2015)141

364. Anisimov A, Di Bari P. Cold dark matter from heavy right-handed neutrino mixing. Phys Rev. (2009) D80:073017. doi: 10.1103/PhysRevD.80.073017

365. De Roeck A, Ellis J, Grojean C, Heinemeyer S, Jakobs K, Weiglein G, et al. From the LHC to Future Colliders. Eur Phys J. (2010) C66:525-83. doi: 10.1140/epjc/s10052-010-1244-3

366. Aarons G, Djouadi A, Lykken J, Moenig K, Okada Y, Oreglia M, et al. International Linear Collider. Reference Design Report. Vol. 2. physics at the ILC. arXiv:0709.1893 (2007).

367. Baer H, Barklow T, Fujii K, Gao Y, Hoang A, Kanemura S, et al. The International Linear Collider. Technical Design Report. Vol. 2. Physics. (2013). doi: 10.2172/1095028

368. Linssen L, Miyamoto A, Stanitzki M, Weerts H. Physics and Detectors at CLIC: CLIC Conceptual Design Report. arXiv:1202.5940 (2012).

369. Haber HE, Kane GL. The search for supersymmetry: probing physics beyond the standard model. Phys Rept. (1985) 117:75-263. doi: 10.1016/0370-1573(85)90051-1

370. Borschensky C, Krämer M, Kulesza A, Mangano M, Padhi S, Plehn $\mathrm{T}$, et al. Squark and gluino production cross sections in pp collisions at $\sqrt{s}=13,14,33$ and $100 \mathrm{TeV}$. Eur Phys J. (2014) C74:3174. doi: 10.1140/epjc/s10052-014-3174-y

371. Beenakker W, Borschensky C, Krämer M, Kulesza A, Laenen E. NNLLfast: predictions for coloured supersymmetric particle production at the LHC with threshold and Coulomb resummation. J High Energy Phys. (2016) 12:133. doi: 10.1007/JHEP12(2016)133

372. Aaboud M, Abbott B, Abdinov O, Abeloos B, Abidi SH, AbouZeid O, et al. Search for squarks and gluinos in final states with jets and missing transverse momentum using $36 \mathrm{fb}^{-1}$ of $\sqrt{s}=13 \mathrm{TeV}$ pp collision data with the ATLAS detector. Phys Rev. (2018) D97:112001. doi: 10.1103/PhysRevD.97.112001

373. Sirunyan AM, Tumasyan A, Adam W, Ambrogi F, Asilar E, Bergauer $\mathrm{T}$, et al. Search for natural and split supersymmetry in proton-proton collisions at $\sqrt{s}=13 \mathrm{TeV}$ in final states with jets and missing transverse momentum. J High Energy Phys. (2018) 05:025. doi: 10.1007/ JHEP05(2018)025

374. Sirunyan AM, Tumasyan A, Adam W, Ambrogi F, Asilar E, Bergauer T, et al. Search for supersymmetry in multijet events with missing transverse momentum in proton-proton collisions at $13 \mathrm{TeV}$. Phys Rev. (2017) D96:032003. doi: 10.1103/PhysRevD.96.032003

375. Aaboud M, Aad G, Abbott B, Abdinov O, Abeloos B, Abidi SH, et al. Search for squarks and gluinos in events with an isolated lepton, jets, and missing transverse momentum at $\sqrt{s}=13 \mathrm{TeV}$ with the ATLAS detector. Phys Rev. (2017) D96:112010. doi: 10.1103/PhysRevD.96.112010

376. Sirunyan AM, Tumasyan A, Adam W, Ambrogi F, Asilar E, Bergauer T, et al. Search for supersymmetry in events with one lepton and multiple jets exploiting the angular correlation between the lepton and the missing transverse momentum in proton-proton collisions at $\sqrt{s}=13 \mathrm{TeV}$. Phys Lett. (2018) B780:384-409. doi: 10.1016/j.physletb.2018.03.028
377. Sirunyan AM, Tumasyan A, Adam W, Ambrogi F, Asilar E, Bergauer T, et al. Search for Supersymmetry in $p p$ Collisions at $\sqrt{s}=13 \mathrm{TeV}$ in the SingleLepton Final State Using the Sum of Masses of Large-Radius Jets. Phys Rev Lett. (2017) 119:151802. doi: 10.1103/PhysRevLett.119.151802

378. Aaboud M, Georges Aad (Marseille C, Abdinov O, Abeloos B, Abidi SH, et al. Search for new phenomena using the invariant mass distribution of same-flavour opposite-sign dilepton pairs in events with missing transverse momentum in $\sqrt{s}=13 \mathrm{TeV}$ pp collisions with the ATLAS detector. Eur Phys J. (2018) C78:625. doi: 10.1140/epjc/s10052-018-6081-9

379. Sirunyan AM, Tumasyan A, Adam W, Ambrogi F, Asilar E, Bergauer T, et al. Search for top squarks and dark matter particles in opposite-charge dilepton final states at $\sqrt{s}=13 \mathrm{TeV}$. Phys Rev. (2018) D97:032009. doi: 10.1103/PhysRevD.97.032009

380. Aaboud M, Aad G, Abbott B, Abdinov O, Abeloos B, Abidi SH, et al. Search for supersymmetry in final states with two same-sign or three leptons and jets using $36 \mathrm{fb}^{-1}$ of $\sqrt{s}=13 \mathrm{TeV} p p$ collision data with the ATLAS detector. J High Energy Phys. (2017) 09:084. doi: 10.1007/JHEP09 (2017)084

381. Sirunyan AM, Tumasyan A, Adam W, Ambrogi F, Asilar E, Bergauer T, et al. Search for supersymmetry in events with at least three electrons or muons, jets, and missing transverse momentum in proton-proton collisions at $\sqrt{s}=13 \mathrm{TeV}$. J High Energy Phys. (2018) 02:067. doi: 10.1007/jhep02 (2018)067

382. Aaboud M, Aad G, Abbott B, Abdinov O, Abeloos B, Abidi SH, et al. Search for supersymmetry in final states with missing transverse momentum and multiple $b$-jets in proton-proton collisions at $\sqrt{s}=13 \mathrm{TeV}$ with the ATLAS detector. J High Energy Phys. (2018) 06:107. doi: 10.1007/JHEP06 (2018) 107

383. Sirunyan AM, Tumasyan A, Adam W, Ambrogi F, Asilar E, Bergauer $\mathrm{T}$, et al. Search for physics beyond the standard model in events with high-momentum higgs bosons and missing transverse momentum in proton-proton collisions at $13 \mathrm{TeV}$. Phys Rev Lett. (2018) 120:241801. doi: 10.1103/PhysRevLett.120.241801

384. Sirunyan AM, Tumasyan A, Adam W, Ambrogi F, Asilar E, Bergauer $\mathrm{T}$, et al. Search for supersymmetry in proton-proton collisions at $13 \mathrm{TeV}$ using identified top quarks. Phys Rev. (2018) D97:012007. doi: 10.1103/PhysRevD.97.012007

385. Aaboud M, Aad G, Abbott B, Abdinov O, Abeloos B, Abhayasinghe DK, et al. Search for squarks and gluinos in final states with hadronically decaying $\tau$-leptons, jets, and missing transverse momentum using $p p$ collisions at $\sqrt{s}=13 \mathrm{TeV}$ with the ATLAS detector. Phys Rev. (2018) 99:012009. doi: 10.1103/PhysRevD.99.012009

386. Aaboud M, Aad G, Abbott B, Abdinov O, Abeloos B, Abidi SH, et al. Search for a scalar partner of the top quark in the jets plus missing transverse momentum final state at $\sqrt{s}=13 \mathrm{TeV}$ with the ATLAS detector. J High Energy Phys. (2017) 12:085. doi: 10.1007/JHEP12(2017)085

387. Sirunyan AM, Tumasyan A, Adam W, Ambrogi F, Asilar E, Bergauer T, et al. Search for top squark pair production in pp collisions at $\sqrt{s}=$ $13 \mathrm{TeV}$ using single lepton events. J High Energy Phys. (2017) 10:019. doi: 10.1007/JHEP10(2017)019

388. Aaboud M, Aad G, Abbott B, Abdinov O, Abeloos B, Abidi SH, et al. Search for supersymmetry in events with $b$-tagged jets and missing transverse momentum in $p p$ collisions at $\sqrt{s}=13 \mathrm{TeV}$ with the ATLAS detector. J High Energy Phys. (2017) 11:195. doi: 10.1007/JHEP11(2017)195

389. Sirunyan AM, Tumasyan A, Adam W, Ambrogi F, Asilar E, Bergauer $\mathrm{T}$, et al. Search for the pair production of third-generation squarks with two-body decays to a bottom or charm quark and a neutralino in proton-proton collisions at $\sqrt{s}=13 \mathrm{TeV}$. Phys Lett. (2018) B778:263-91. doi: 10.1016/. physletb.2018.01.012

390. Aaboud M, Aad G, Abbott B, Abdinov O, Abeloos B, Abidi SH, et al. Search for top-squark pair production in final states with one lepton, jets, and missing transverse momentum using $36 \mathrm{fb}^{-1}$ of $\sqrt{s}=13 \mathrm{TeV}$ pp collision data with the ATLAS detector. J High Energy Phys. (2018) 06:108. doi: 10.1007/JHEP09(2018)050

391. Sirunyan AM, Tumasyan A, Adam W, Ambrogi F, Asilar E, Bergauer T, et al. Search for top squarks decaying via four-body or chargino-mediated modes in single-lepton final states in proton-proton collisions at $\sqrt{s}=13 \mathrm{TeV}$. $J$ High Energy Phys. (2018) 09:065. doi: 10.1007/JHEP09(2018)065 
392. Aaboud M, Aad G, Abbott B, Abdinov O, Abeloos B, Abidi SH, et al. Search for direct top squark pair production in final states with two leptons in $\sqrt{s}=13 \mathrm{TeV} p p$ collisions with the ATLAS detector. Eur Phys J. (2017) C77:898.

393. Sirunyan AM, Tumasyan A, Adam W, Ambrogi F, Asilar E, Bergauer T, et al. Searches for pair production of charginos and top squarks in final states with two oppositely charged leptons in proton-proton collisions at $\sqrt{s}=13 \mathrm{TeV}$. J High Energy Phys. (2018) 11:079. doi: 10.1007/JHEP11(2018)079

394. Aaboud M, Aad G, Abbott B, Abdinov O, Abeloos B, Abidi SH, et al. Search for direct top squark pair production in events with a Higgs or $Z$ boson, and missing transverse momentum in $\sqrt{s}=13 \mathrm{TeV} p p$ collisions with the ATLAS detector. J High Energy Phys. (2017) 08:006. doi: 10.1007/JHEP11 (2017) 195

395. Aaboud M, Aad G, Abbott B, Abdinov O, Abeloos B, Abidi SH, et al. Search for electroweak production of supersymmetric states in scenarios with compressed mass spectra at $\sqrt{s}=13 \mathrm{TeV}$ with the ATLAS detector. Phys Rev. (2018) D97:052010. doi: 10.1103/PhysRevD.97.052010

396. Sirunyan AM, Tumasyan A, Adam W, Ambrogi F, Asilar E, Bergauer $\mathrm{T}$, et al. Search for supersymmetric partners of electrons and muons in proton-proton collisions at $\sqrt{s}=13 \mathrm{TeV}$. Phys Lett. (2018) B790:140-166. doi: 10.1016/j.physletb.2019.01.005

397. Sirunyan AM, Tumasyan A, Adam W, Ambrogi F, Asilar E, Bergauer T, et al. Combined search for electroweak production of charginos and neutralinos in proton-proton collisions at $\sqrt{s}=13 \mathrm{TeV}$. J High Energy Phys. (2018) 03:160. doi: 10.1007/JHEP03(2018)160

398. Aaboud M, Aad G, Abbott B, Abdinov O, Abeloos B, Abidi SH, et al. Search for electroweak production of supersymmetric particles in final states with two or three leptons at $\sqrt{s}=13 \mathrm{TeV}$ with the ATLAS detector. (2018) C78:995. doi: 10.1140/epjc/s10052-018-6423-7

399. Aaboud M, Aad G, Abbott B, Abdinov O, Abeloos B, Abhayasinghe DK, et al. Search for chargino-neutralino production using recursive jigsaw reconstruction in final states with two or three charged leptons in protonproton collisions at $\sqrt{s}=13 \mathrm{TeV}$ with the ATLAS detector. Phys Rev. (2018) D98:092012. doi: 10.1103/PhysRevD.98.092012

400. Aaboud M, Aad G, Abbott B, Abdinov O, Abeloos B, Abidi SH, et al. Search for supersymmetry in events with four or more leptons in $\sqrt{s}=13 \mathrm{TeV} p p$ collisions with ATLAS. Phys Rev. (2018) D98:032009. doi: 10.1103/PhysRevD.98.032009

401. Aaboud M, Aad G, Abbott B, Abdinov O, Abeloos B, Abidi SH, et al. Search for the direct production of charginos and neutralinos in final states with tau leptons in $\sqrt{s}=13 \mathrm{TeV} p p$ collisions with the ATLAS detector. Eur Phys J. (2018) C78:154. doi: 10.1140/epjc/s10052-018-5583-9

402. Sirunyan AM, Tumasyan A, Adam W, Ambrogi F, Asilar E, Bergauer T, et al. Search for supersymmetry in events with a $\tau$ lepton pair and missing transverse momentum in proton-proton collisions at $\sqrt{s}=13 \mathrm{TeV}$. J High Energy Phys. (2018) 11:151. doi: 10.1007/JHEP11(2018)151

403. Aaboud M, Aad G, Abbott B, Abdinov O, Abeloos B, Abidi SH, et al. Search for pair production of higgsinos in final states with at least three $b$-tagged jets in $\sqrt{s}=13 \mathrm{TeV} p p$ collisions using the ATLAS detector. Phys Rev. (2018) D98:092002. doi: 10.1140/epjc/s10052-018-6457-x

404. Aaboud M, Aad G, Abbott B, Abdinov O, Abeloos B, Abhayasinghe DK, et al. Search for Higgs boson pair production in the $W W^{(*)} W W^{(*)}$ decay channel using ATLAS data recorded at $\sqrt{s}=13 \mathrm{TeV}$. arXiv:1811.11028 (2018).

405. Aaboud M, Aad G, Abbott B, Abdinov O, Abeloos B, Abidi SH, et al. Search for heavy ZZ resonances in the $\ell^{+} \ell^{-} \ell^{+} \ell^{-}$and $\ell^{+} \ell^{-} \nu \bar{v}$ final states using proton-proton collisions at $\sqrt{s}=13 \mathrm{TeV}$ with the ATLAS detector. Eur Phys J. (2018) C78:293. doi: 10.1140/epjc/s10052-018-5686-3

406. Aaboud M, Aad G, Abbott B, Abdinov O, Abeloos B, Abidi SH, et al, Search for heavy resonances decaying into $W W$ in the ev $\mu \nu$ final state in pp collisions at $\sqrt{s}=13 \mathrm{TeV}$ with the ATLAS detector. Eur Phys J. (2018) C78:24. doi: 10.1140/epjc/s10052-017-5491-4

407. Sirunyan AM, Tumasyan A, Adam W, Ambrogi F, Asilar E, Bergauer T, et al. A search for pair production of new light bosons decaying into muons in proton-proton collisions at $13 \mathrm{TeV}$. arXiv:1812.00380 (2018).

408. Aaboud M, Aad G, Abbott B, Abdinov O, Abeloos B, Abidi SH, et al. Search for pair production of Higgs bosons in the $b \bar{b} b \bar{b}$ final state using protonproton collisions at $\sqrt{s}=13 \mathrm{TeV}$ with the ATLAS detector. J High Energy Phys. (2018) 01:030. doi: 10.1007/JHEP01(2019)030
409. Sirunyan AM, Tumasyan A, Adam W, Ambrogi F, Asilar E, Bergauer T, et al. Search for beyond the standard model Higgs bosons decaying into a $\mathrm{b} \overline{\mathrm{b}}$ pair in pp collisions at $\sqrt{s}=13 \mathrm{TeV}$. J High Energy Phys. (2018) 08:113. doi: 10.1007/JHEP08(2018)113

410. Aaboud M, Aad G, Abbott B, Abdinov O, Abeloos B, Abhayasinghe DK, et al. Search for Higgs boson pair production in the $b \bar{b} W W^{*}$ decay mode at $\sqrt{s}=13 \mathrm{TeV}$ with the ATLAS detector. J High Energy Phys. (2018) 04:092 . doi: 10.1007/JHEP04(2019)092

411. Aaboud M, Aad G, Abbott B, Abdinov O, Abeloos B, Abhayasinghe DK, et al. Search for Higgs boson pair production in the $\gamma \gamma b \bar{b}$ final state with $13 \mathrm{TeV}$ $p p$ collision data collected by the ATLAS experiment. J High Energy Phys. (2018) 11:040. doi: 10.1007/JHEP11(2018)040

412. Sirunyan AM, Tumasyan A, Adam W, Ambrogi F, Asilar E, Bergauer $\mathrm{T}$, et al. Search for a standard model-like Higgs boson in the mass range between 70 and $110 \mathrm{GeV}$ in the diphoton final state in protonproton collisions at $\sqrt{s}=8$ and $13 \mathrm{TeV}$. Phys Lett. (2018) B793:320-47. doi: 10.1016/j.physletb.2019.03.064

413. Aaboud M, Aad G, Abbott B, Abdinov O, Abeloos B, Abhayasinghe DK, et al. Search for Resonant and Nonresonant Higgs Boson Pair Production in the $b \bar{b} \tau^{+} \tau^{-}$Decay Channel in $p p$ Collisions at $\sqrt{s}=$ $13 \mathrm{TeV}$ with the ATLAS Detector. Phys Rev Lett. (2018) 121:191801. doi: 10.1103/PhysRevLett.121.191801

414. Khachatryan V, Sirunyan AM, Tumasyan A, Wolfgang Adam EA, Bergauer $\mathrm{T}$, et al. Search for light bosons in decays of the $125 \mathrm{GeV}$ Higgs boson in proton-proton collisions at $\sqrt{s}=8 \mathrm{TeV}$. J High Energy Phys. (2017) 10:076. doi: 10.1007/JHEP10(2017)076

415. Sirunyan AM, Tumasyan A, Adam W, Ambrogi F, Asilar E, Bergauer T, et al. Search for a light pseudoscalar Higgs boson produced in association with bottom quarks in pp collisions at $\sqrt{s}=8 \mathrm{TeV}$. J High Energy Phys. (2017) 11:010. doi: 10.1007/JHEP11(2017)010

416. Aaboud M, Aad G, Abbott B, Abdinov O, Abeloos B, Abhayasinghe DK, et al. Search for charged Higgs bosons decaying into top and bottom quarks at $\sqrt{s}=13 \mathrm{TeV}$ with the ATLAS detector. J High Energy Phys. (2018) 11:085. doi: 10.1007/JHEP11(2018)085

417. Aaboud M, Aad G, Abbott B, Abdinov O, Abeloos B, Abidi SH, et al. Search for additional heavy neutral Higgs and gauge bosons in the ditau final state produced in $36 \mathrm{fb}^{-1}$ of pp collisions at $\sqrt{s}=13 \mathrm{TeV}$ with the ATLAS detector. J High Energy Phys. (2018) 01:055. doi: 10.1007/JHEP11 (2018)051

418. Sirunyan AM, Tumasyan A, Adam W, Ambrogi F, Asilar E, Bergauer T, et al. Search for additional neutral MSSM Higgs bosons in the $\tau \tau$ final state in proton-proton collisions at $\sqrt{s}=13 \mathrm{TeV}$. J High Energy Phys. (2018) 09:007. doi: 10.1007/JHEP09(2018)007

419. Aaboud M, Aad G, Abbott B, Abdinov O, Abeloos B, Abidi SH, et al. Search for new phenomena in high-mass diphoton final states using $37 \mathrm{fb}^{-1}$ of proton-proton collisions collected at $\sqrt{s}=13 \mathrm{TeV}$ with the ATLAS detector. Phys Lett. (2017) B775:105-25. doi: 10.1016/j.physletb.2017.10.039

420. Aaboud M, Aad G, Abbott B, Abdinov O, Abeloos B, Abhayasinghe DK, et al. Search for Higgs boson pair production in the $\gamma \gamma W W^{*}$ channel using $p p$ collision data recorded at $\sqrt{s}=13 \mathrm{TeV}$ with the ATLAS detector. Eur Phys J. (2018) C78:1007. doi: 10.1103/PhysRevD.98.092002

421. Aaboud M, Aad G, Abbott B, Abdinov O, Abeloos B, Abhayasinghe DK, et al. A search for pairs of highly collimated photon-jets in $p p$ collisions at $\sqrt{s}=13 \mathrm{TeV}$ with the ATLAS detector. Phys Rev. (2018) D99:012008. doi: 10.1103/PhysRevD.99.012008

422. Aad G, Abbott B, Abdallah J, Abdinov O, Aben R, Abolins M, et al. Search for a charged higgs boson produced in the vector-boson fusion mode with decay $H^{ \pm} \rightarrow W^{ \pm} Z$ Using $p p$ collisions at $\sqrt{s}=8 \mathrm{TeV}$ with the ATLAS experiment. Phys Rev Lett. (2015) 114:231801. doi: 10.1103/PhysRevLett.114.231801

423. Sirunyan AM, Tumasyan A, Adam W, Ambrogi F, Asilar E, Bergauer T, et al. Search for Charged Higgs Bosons Produced via Vector Boson Fusion and Decaying into a Pair of $W$ and $Z$ Bosons Using $p p$ Collisions at $\sqrt{s}=13 \mathrm{TeV}$. Phys Rev Lett. (2017) 119:141802. doi: 10.1103/PhysRevLett.119.141802

424. Sirunyan AM, Tumasyan A, Adam W, Ambrogi F, Asilar E, Bergauer T, et al. Search for a charged Higgs boson decaying to charm and bottom quarks in proton-proton collisions at $\sqrt{s}=8 \mathrm{TeV}$. J High Energy Phys. (2018) 11:115. doi: 10.1007/JHEP11(2018)115 
425. Aaboud M, Aad G, Abbott B, Abdallah J, Abdinov O, Abeloos B, et al. Search for charged Higgs bosons produced in association with a top quark and decaying via $H^{ \pm} \rightarrow \tau v$ using $p p$ collision data recorded at $\sqrt{s}=13 \mathrm{TeV}$ by the ATLAS detector. Phys Lett. (2016) B759:555-74. doi: 10.1016/j.physletb.2016.06.017

426. Aaboud M, Aad G, Abbott B, Abdinov O, Abeloos B, Abidi SH, et al. Search for R-parity-violating supersymmetric particles in multi-jet final states produced in $p$ - $p$ collisions at $\sqrt{s}=13 \mathrm{TeV}$ using the ATLAS detector at the LHC. Phys Lett. (2018) B785:136-58. doi: 10.1016/j.physletb.2018. 08.021

427. Aaboud M, Aad G, Abbott B, Abdinov O, Abeloos B, Abidi SH, et al. Search for new phenomena in a lepton plus high jet multiplicity final state with the ATLAS experiment using $\sqrt{s}=13 \mathrm{TeV}$ proton-proton collision data. J High Energy Phys. (2017) 09:088. doi: 10.1007/JHEP09(2017)088

428. Aaboud M, Aad G, Abbott B, Abdinov O, Abeloos B, Abidi SH, et al. A search for pair-produced resonances in four-jet final states at $\sqrt{s}=13 \mathrm{TeV}$ with the ATLAS detector. Eur Phys J. (2018) C78:250. doi: 10.1140/epjc/s10052-018-5693-4

429. Sirunyan AM, Tumasyan A, Adam W, Ambrogi F, Asilar E, Bergauer $\mathrm{T}$, et al. Search for $R$-parity violating supersymmetry in pp collisions at $\sqrt{s}=13 \mathrm{TeV}$ using b jets in a final state with a single lepton, many jets, and high sum of large-radius jet masses. Phys Lett. (2018) B783:114-39. doi: 10.1016/j.physletb.2018.06.028

430. Aaboud M, Aad G, Abbott B, Abdinov O, Abeloos B, Abidi SH, et al. Search for B-L R -parity-violating top squarks in $\sqrt{s}=13 \mathrm{TeV}$ pp collisions with the ATLAS experiment. Phys Rev. (2018) D97:032003. doi: 10.1103/PhysRevD.97.032003

431. Sirunyan AM, Tumasyan A, Adam W, Ambrogi F, Asilar E, Bergauer T, et al. Search for resonant production of second-generation sleptons with samesign dimuon events in proton-proton collisions at $\sqrt{s}=13 \mathrm{TeV}$. Eur Phys J. (2018) C79:305. doi: 10.1140/epjc/s10052-019-6800-x

432. Aaboud M, Aad G, Abbott B, Abdinov O, Abeloos B, Abhayasinghe DK, et al. Search for long-lived particles produced in $p p$ collisions at $\sqrt{s}=13 \mathrm{TeV}$ that decay into displaced hadronic jets in the ATLAS muon spectrometer. Phys Rev. (2018) D99:052005. doi: 10.1103/PhysRevD.99.052005

433. Aaboud M, Aad G, Abbott B, Abdinov O, Abeloos B, Abhayasinghe DK, et al. Search for heavy charged long-lived particles in proton-proton collisions at $\sqrt{s}=13 \mathrm{TeV}$ using an ionisation measurement with the ATLAS detector. Phys Lett. (2019) B788:96-116. doi: 10.1016/j.physletb.2018.10.055

434. Aaboud M, Aad G, Abbott B, Abdinov O, Abeloos B, Abidi SH, et al. Search for long-lived, massive particles in events with displaced vertices and missing transverse momentum in $\sqrt{s}=13 \mathrm{TeV} p p$ collisions with the ATLAS detector. Phys Rev. (2018) D97:052012. doi: 10.1103/PhysRevD.97.052012

435. Aaboud M, Aad G, Abbott B, Abdinov O, Abeloos B, Abidi SH, et al. Search for long-lived charginos based on a disappearing-track signature in pp collisions at $\sqrt{s}=13 \mathrm{TeV}$ with the ATLAS detector. J High Energy Phys. (2018) 06:022. doi: 10.1007/JHEP06(2018)022

436. Aaboud M, Aad G, Abbott B, Abdinov O, Abeloos B, Abhayasinghe DK, et al. Search for long-lived particles in final states with displaced dimuon vertices in $p p$ collisions at $\sqrt{s}=13 \mathrm{TeV}$ with the ATLAS detector. Phys Rev D. (2018) 99:012001. doi: 10.1103/PhysRevD.99.012001

437. Sirunyan AM, Tumasyan A, Adam W, Ambrogi F, Asilar E, Bergauer T, et al. Search for new long-lived particles at $\sqrt{s}=13 \mathrm{TeV}$. Phys Lett. (2018) B780:432-54. doi: 10.1016/j.physletb.2018.03.019

438. Sirunyan AM, Tumasyan A, Adam W, Ambrogi F, Asilar E, Bergauer $\mathrm{T}$, et al. Search for long-lived particles decaying into displaced jets in proton-proton collisions at $\sqrt{s}=13 \mathrm{TeV}$. Phys Rev. (2018) D99:032011. doi: 10.1103/PhysRevD.99.032011

439. Sirunyan AM, Tumasyan A, Adam W, Ambrogi F, Asilar E, Bergauer T, et al. Search for long-lived particles with displaced vertices in multijet events in proton-proton collisions at $\sqrt{s}=13 \mathrm{TeV}$. Phys Rev. (2018) D98:092011. doi: 10.1103/PhysRevD.98.092011

440. Sirunyan AM, Tumasyan A, Adam W, Ambrogi F, Asilar E, Bergauer T, et al. Search for gauge-mediated supersymmetry in events with at least one photon and missing transverse momentum in pp collisions at $\sqrt{s}=13 \mathrm{TeV}$. Phys Lett. (2018) B780:118-43. doi: 10.1016/j.physletb.2018.02.045

441. Sirunyan AM, Tumasyan A, Adam W, Ambrogi F, Asilar E, Bergauer $\mathrm{T}$, et al. Search for supersymmetry in events with at least one photon, missing transverse momentum, and large transverse event activity in protonproton collisions at $\sqrt{s}=13 \mathrm{TeV}$. J High Energy Phys. (2017) 12:142. doi: 10.1007/JHEP12(2017)142

442. Aaboud M, Aad G, Abbott B, Abdallah J, Abdinov O, Abeloos B, et al. Search for supersymmetry in a final state containing two photons and missing transverse momentum in $\sqrt{s}=13 \mathrm{TeV} p p$ collisions at the LHC using the ATLAS detector. Eur Phys J. (2016) C76:517. doi: 10.1140/epjc/s10052-016-4344-x

443. Aaboud M, Aad G, Abbott B, Abdinov O, Abeloos B, Abidi SH, et al. Search for photonic signatures of gauge-mediated supersymmetry in 13 $\mathrm{TeV} p p$ collisions with the ATLAS detector. Phys Rev. (2018) D97:092006. doi: 10.1103/PhysRevD.97.092006

444. Sirunyan AM, Tumasyan A, Adam W, Ambrogi F, Asilar E, Bergauer $\mathrm{T}$, et al. Search for Higgsino pair production in $p p$ collisions at $\sqrt{s}=$ $13 \mathrm{TeV}$ in final states with large missing transverse momentum and two Higgs bosons decaying via $H \rightarrow b \bar{b}$. Phys Rev. (2018) D97:032007. doi: 10.1103/PhysRevD.97.032007

445. Aaboud M, Aad G, Abbott B, Abdinov O, Abeloos B, Abidi SH, et al. Search for top squarks decaying to tau sleptons in $p p$ collisions at $\sqrt{s}=13 \mathrm{TeV}$ with the ATLAS detector. Phys Rev. (2018) D98:032008. doi: 10.1103/PhysRevD.98.032008

446. Heister A, Schael S, Barate R, Bruneliere R, Bonis ID, Decamp D, et al. Search for charginos nearly mass degenerate with the lightest neutralino in e+ e- collisions at center-of-mass energies up to 209-GeV. Phys Lett. (2002) B533:223-36. doi: 10.1016/S0370-2693(02)01584-8

447. Heister A, Schael S, Barate R, Bruneliere R, Bonis ID, Decamp D, et al. Absolute lower limits on the masses of selectrons and sneutrinos in the MSSM. Phys Lett. (2002) B544:73-88. doi: 10.1016/S0370-2693(02)02471-1

448. Abbiendi G, Ainsley C, Akesson PF, Alexander G, Allison J, Amaral P, et al. Search for chargino and neutralino production at $s^{(1 / 2)}=192-\mathrm{GeV}$ to 209 GeV at LEP. Eur Phys J. (2004) C35:1-20. doi: 10.1140/epjc/s2004-01758-8

449. Abbiendi G, Ainsley C, Akesson PF, Alexander G, Allison J, Amaral P, et al. Search for anomalous production of dilepton events with missing transverse momentum in e+ e- collisions at $\mathrm{s}^{(1 / 2)}=183-\mathrm{Gev}$ to 209-GeV. Eur Phys J. (2004) C32:453-73. doi: 10.1140/epjc/s2003-01466-y

450. Acciarri M, Achard P, Adriani O, Aguilar-Benitez M, Alcaraz J, Alemanni G, et al. Search for charginos and neutralinos in $e^{+} e^{-}$collisions at $\sqrt{S}=189-$ GeV. Phys Lett. (2000) B472:420-33. doi: 10.1016/S0370-2693(99)01388-X

451. Achard P, Adriani O, Aguilar-Benitez M, Alcaraz J, Alemanni G, Allaby J, et al. Search for scalar leptons and scalar quarks at LEP. Phys Lett. (2004) B580:37-49. doi: 10.1016/j.physletb.2003.10.010

452. Abdallah J, Abreu P, Adam W, Adzic P, Albrecht T, Alderweireld T, et al. Searches for supersymmetric particles in e+ e- collisions up to $208-\mathrm{GeV}$ and interpretation of the results within the MSSM. Eur Phys J. (2003) C31:421-79. doi: 10.1140/epjc/s2003-01355-5

453. Roszkowski L, Ruiz de Austri R, Trotta R, Tsai YLS, Varley TA. Global fits of the Non-Universal Higgs Model. Phys Rev. (2011) D83:015014. doi: 10.1103/PhysRevD.83.015014

454. Bertone G, Cerdeno DG, Fornasa M, Ruiz de Austri R, Strege C, Trotta R. Global fits of the cMSSM including the first LHC and XENON100 data. J Cosmol Astropart Phys. (2012) 1201:015. doi: 10.1088/1475-7516/2012/01/015

455. Bechtle P, Bringmann T, Desch K, Dreiner H, Hamer M, Hensel C, et al. Constrained Supersymmetry after two years of LHC data: a global view with Fittino. J High Energy Phys. (2012) 06:098. doi: 10.1007/JHEP06 (2012)098

456. Buchmueller O, Cavanaugh R, Roeck AD, Dolan MJ, Ellis JR, Flacher H, et al. The CMSSM and NUHM1 after LHC Run 1. Eur Phys J. (2014) C74:2922. doi: 10.1140/epjc/s10052-014-2922-3

457. Buchmueller O, Cavanaugh R, Citron M, Roeck AD, Dolan MJ, Ellis JR, et al. The NUHM2 after LHC Run 1. Eur Phys J. (2014) C74:3212. doi: 10.1140/epjc/s10052-014-3212-9

458. Han C, Hikasa Ki, Wu L, Yang JM, Zhang Y. Status of CMSSM in light of current LHC Run-2 and LUX data. Phys Lett. (2017) B769:470-76. doi: 10.1016/j.physletb.2017.04.026

459. Athron P, Balázs C, Bringmann T, Buckley A, Chrzạszcz M, Conrad J, et al. Global fits of GUT-scale SUSY models with GAMBIT. Eur Phys J. (2017) C77:824. doi: 10.1140/epjc/s10052-017-5167-0 
460. Bagnaschi E, Sakurai K, Borsato M, Buchmueller O, Citron M, Costa JC, et al. Likelihood Analysis of the pMSSM11 in Light of LHC 13-TeV Data. Eur Phys J. (2018) C78:256. doi: 10.1140/epjc/s10052-018-5697-0

461. Athron P, Balázs C, Bringmann T, Buckley A, Chrząszcz M, Conrad J, et al. A global fit of the MSSM with GAMBIT. Eur Phys J. (2017) C77:879. doi: 10.1140/epjc/s10052-017-5196-8

462. Bertone G, Calore F, Caron S, Ruiz R, Kim JS, Trotta R, et al. Global analysis of the pMSSM in light of the Fermi GeV excess: prospects for the LHC RunII and astroparticle experiments. J Cosmol Astropart Phys. (2016) 1604:037. doi: $10.1088 / 1475-7516 / 2016 / 04 / 037$

463. Bagnaschi E, Costa JC, Sakurai K, Borsato M, Buchmueller O, Cavanaugh R, et al. Likelihood Analysis of Supersymmetric SU(5) GUTs. Eur Phys J. (2017) C77:104. doi: 10.1140/epjc/s10052-017-4639-6

464. Arbuzov AB, Awramik M, Czakon M, Freitas A, Grunewald MW, Monig K, et al. ZFITTER: A Semi-analytical program for fermion pair production in e+e- annihilation, from version 6.21 to version 6.42. Comput Phys Commun. (2006) 174:728-58. doi: 10.1016/j.cpc.2005.12.009

465. Ruiz de Austri R, Trotta R, Roszkowski L. A markov chain monte carlo analysis of the CMSSM. J High Energy Phys. (2006) 05:002. doi: 10.1088/1126-6708/2006/05/002

466. Trotta R, Feroz F, Hobson MP, Roszkowski L, Ruiz de Austri R. The Impact of priors and observables on parameter inferences in the Constrained MSSM. J High Energy Phys. (2008) 12:024. doi: 10.1088/1126-6708/2008/ $12 / 024$

467. Bechtle P, Desch K, Wienemann P. Fittino, a program for determining MSSM parameters from collider observables using an iterative method. Comput Phys Commun. (2006) 174:47-70. doi: 10.1016/j.cpc.2005.09.002

468. Buchmueller O, Cavanaugh R, De Roeck A, Ellis JR, Flacher H, Heinemeyer $S$, et al. Likelihood Functions for Supersymmetric Observables in Frequentist Analyses of the CMSSM and NUHM1. Eur Phys J. (2009) C64:391-415. doi: 10.1140/epjc/s10052-009-1159-z

469. Athron P, Balazs C, Bringmann T, Buckley A, Chrzạszcz M, et al. GAMBIT: The Global and Modular Beyond-the-Standard-Model Inference Tool. Eur Phys J. (2017) C77:784. doi: 10.1140/epjc/s10052-017-5321-8

470. Athron P, Balázs C, Dal LA, Edsjö J, Farmer B, Gonzalo TE, et al. SpecBit, DecayBit and PrecisionBit: GAMBIT modules for computing mass spectra, particle decay rates and precision observables. Eur Phys J. (2018) C78:22. doi: 10.1140/epjc/s10052-017-5390-8

471. Bringmann T, Conrad J, Cornell JM, Dal LA, Edsjö J, Farmer B, et al. DarkBit: a GAMBIT module for computing dark matter observables and likelihoods. Eur Phys J. (2017) C77:831. doi: 10.1140/epjc/s10052-017-5155-4

472. Martinez GD, McKay J, Farmer B, Scott P, Roebber E, Putze A, et al. Comparison of statistical sampling methods with ScannerBit, the GAMBIT scanning module. Eur Phys J. (2017) C77:761. doi: 10.1140/epjc/s10052-017-5274-y

473. Balázs C, Buckley A, Dal LA, Farmer B, Jackson P, Krislock A, et al. ColliderBit: a GAMBIT module for the calculation of highenergy collider observables and likelihoods. Eur Phys J. (2017) C77:795. doi: 10.1140/epjc/s10052-017-5285-8

474. Bernlochner FU, Chrzaszcz M, Dal LA, Farmer B, Jackson P, Kvellestad A, et al. FlavBit: A GAMBIT module for computing flavour observables and likelihoods. Eur Phys J. (2017) C77:786. doi: 10.1140/epjc/s10052-017-5157-2

475. Athron P, Balázs C, Bringmann T, Buckley A, Chrzạszcz M, Conrad J, et al. Status of the scalar singlet dark matter model. Eur Phys J. (2017) C77:568. doi: 10.1140/epjc/s10052-0175113-1

476. Athron P, Balázs C, Beniwal A, Bloor S, Camargo-Molina JE, Cornell JM, et al. Global analyses of Higgs portal singlet dark matter models using GAMBIT. Eur Phys J. (2019) C79:38. doi: 10.1140/epjc/s10052-018-6513-6

477. Murayama H, Yanagida T. A viable SU(5) GUT with light leptoquark bosons. Mod Phys Lett. (1992) A7:147-52. doi: 10.1142/S0217732392000070

478. Biggio C, Bordone M, Di Luzio L, Ridolfi G. Massive vectors and loop observables: the $g-2$ case. J High Energy Phys. (2016) 10:002. doi: 10.1007/JHEP10(2016)002

479. Gabrielli E, Marzola L, Raidal M, Veermäe H. Dark matter and spin-1 milli-charged particles. J High Energy Phys. (2015) 08:150. doi: 10.1007/JHEP08(2015)150
480. Doršner I, Fajfer S, Greljo A, Kamenik JF, Košnik N. Physics of leptoquarks in precision experiments and at particle colliders. Phys Rept. (2016) 641:1-68. doi: 10.1016/j.physrep.2016.06.001

481. Buchmuller W, Ruckl R, Wyler D. Leptoquarks in Lepton-Quark Collisions. Phys Lett. (1987) B191:442-8. doi: 10.1016/0370-2693(87)90637-X

482. Davidson S, Bailey DC, Campbell BA. Model independent constraints on leptoquarks from rare processes. Z Phys. (1994) C61:613-44. doi: 10.1007/BF01552629

483. Grifols JA, Mendez A. Pair Production of Colored Hypermesons at Very High-energy. Phys Rev. (1982) D26:324. doi: 10.1103/PhysRevD.26.324

484. Antoniadis I, Baulieu L, Delduc F. Supersymmetric enhancement factor for the one jet cross-section in $p \bar{p}$ Reactions. Z Phys. (1984) C23:119. doi: 10.1007/BF01557587

485. Eichten E, Hinchliffe I, Lane KD, Quigg C. Super Collider Physics. Rev Mod Phys. (1984) 56:579-707. doi: 10.1103/RevModPhys.56.579

486. Altarelli G, Ruckl R. Searching for a Scalar Top at the CERN $p \bar{p}$ Collider. Phys Lett. (1984) 144B:126-32. doi: 10.1016/0370-2693(84)90190-4

487. Dawson S, Eichten E, Quigg C. Search for supersymmetric particles in hadron-hadron collisions. Phys Rev. (1985) D31:1581. doi: 10.1103/PhysRevD.31.1581

488. Blumlein J, Boos E, Kryukov A. Leptoquark pair production in hadronic interactions. Z Phys. (1997) C76:137-53. doi: 10.1007/s0028800 50538

489. Dorsner I, Fajfer S, Greljo A. Cornering Scalar Leptoquarks at LHC. J High Energy Phys. (2014) 10:154. doi: 10.1007/JHEP10(2014)154

490. Doršner I, Greljo A. Leptoquark toolbox for precision collider studies. J High Energy Phys. (2018) 05:126. doi: 10.1007/JHEP05(2018)126

491. Plehn T, Spiesberger H, Spira M, Zerwas PM. Formation and decay of scalar leptoquarks / squarks in e p collisions. Z Phys. (1997) C74:611-4. doi: $10.1007 /$ s002880050426

492. Diaz B, Schmaltz M, Zhong YM. The leptoquark Hunter's guide: Pair production. J High Energy Phys. (2017) 10:097. doi: 10.1007/JHEP10(2017)097

493. Sirunyan AM, Tumasyan A, Adam W, Ambrogi F, Asilar E, Bergauer T, et al. Search for pair production of first-generation scalar leptoquarks at $\sqrt{s}=13$ TeV. Phys Rev. (2018) D99:052002. doi: 10.1103/PhysRevD.99. 052002

494. Aaboud M, Aad G, Abbott B, Abdallah J, Abdinov O, Abeloos B, et al. Search for scalar leptoquarks in pp collisions at $\sqrt{s}=13$ $\mathrm{TeV}$ with the ATLAS experiment. New J Phys. (2016) 18:093016. doi: 10.1088/1367-2630/18/9/093016

495. Aad G, Abbott B, Abdallah J, Abdinov O, Aben R, Abolins M, et al. Searches for scalar leptoquarks in pp collisions at $\sqrt{s}=8 \mathrm{TeV}$ with the ATLAS detector. Eur Phys J. (2016) C76:5. doi: 10.1140/epjc/s10052-0153823-9

496. Khachatryan V, Sirunyan AM, Tumasyan A, Adam W, Asilar E, Bergauer $\mathrm{T}$, et al. Search for heavy neutrinos or third-generation leptoquarks in final states with two hadronically decaying $\tau$ leptons and two jets in protonproton collisions at $\sqrt{s}=13 \mathrm{TeV}$. J High Energy Phys. (2017) 03:077. doi: 10.1007/JHEP03(2017)077

497. Sirunyan AM, Tumasyan A, Adam W, Asilar E, Bergauer T, Brandstetter J, et al. Search for third-generation scalar leptoquarks and heavy righthanded neutrinos in final states with two tau leptons and two jets in protonproton collisions at $\sqrt{s}=13 \mathrm{TeV}$. J High Energy Phys. (2017) 07:121. doi: 10.1007/JHEP07(2017)121

498. Sirunyan AM, Tumasyan A, Adam W, Ambrogi F, Asilar E, Bergauer T, et al. Constraints on models of scalar and vector leptoquarks decaying to a quark and a neutrino at $\sqrt{s}=13 \mathrm{TeV}$. Phys Rev. (2018) D98:032005. doi: 10.1103/PhysRevD.98.032005

499. Sirunyan AM, Tumasyan A, Adam W, Ambrogi F, Asilar E, Bergauer T, et al. Search for third-generation scalar leptoquarks decaying to a top quark and a $\tau$ lepton at $\sqrt{s}=13 \mathrm{TeV}$. Eur Phys J. (2018) C78:707. doi: 10.1140/epjc/s10052-018-6143-z

500. Sirunyan AM, Tumasyan A, Adam W, Ambrogi F, Asilar E, Bergauer T, et al. Search for leptoquarks coupled to third-generation quarks in protonproton collisions at $\sqrt{s}=13 \mathrm{TeV}$. Phys Rev Lett. (2018) 121:241802. doi: 10.1103/PhysRevLett.121.241802 
501. Khachatryan V, Sirunyan AM, Tumasyan A, Adam W, Asilar E, Bergauer $\mathrm{T}$, et al. Search for single production of scalar leptoquarks in protonproton collisions at $\sqrt{s}=8 \mathrm{TeV}$. Phys Rev. (2016) D93:032005. doi: 10.1103/PhysRevD.93.032005

502. Deppisch FF, Bhupal Dev PS, Pilaftsis A. Neutrinos and Collider Physics. New J Phys. (2015) 17:075019. doi: 10.1088/1367-2630/17/7/075019

503. Aaboud M, Aad G, Abbott B, Abdinov O, Abeloos B, Abhayasinghe DK, et al. Search for heavy Majorana or Dirac neutrinos and right-handed $W$ gauge bosons in final states with two charged leptons and two jets at $\sqrt{s}$ = $13 \mathrm{TeV}$ with the ATLAS detector. J High Energy Phys. (2018) 01:016. doi: 10.1007/JHEP01(2019)016

504. Sirunyan AM, Tumasyan A, Adam W, Ambrogi F, Asilar E, Bergauer T, et al. Search for a heavy right-handed $\mathrm{W}$ boson and a heavy neutrino in events with two same-flavor leptons and two jets at $\sqrt{s}=13 \mathrm{TeV}$. J High Energy Phys. (2018) 05:148. doi: 10.1007/JHEP05(2018)148

505. Sirunyan AM, Tumasyan A, Adam W, Ambrogi F, Asilar E, Bergauer T, et al. Search for heavy neutral leptons in events with three charged leptons in proton-proton collisions at $\sqrt{s}=13 \mathrm{TeV}$. Phys Rev Lett. (2018) 120:221801. doi: 10.1103/PhysRevLett.120.221801

506. Drewes M, Garbrecht B. Combining experimental and cosmological constraints on heavy neutrinos. Nucl Phys. (2017) B921:250-315. doi: 10.1016/j.nuclphysb.2017.05.001

507. Bergsma F, Dorenbosch J, Allaby JV, Amaldi U, Barbiellini G, Berger C, et al. A Search for Decays of Heavy Neutrinos in the Mass Range 0.5GeV to 2.8-GeV. Phys Lett. (1986) 166B:473-8. doi: 10.1016/0370-2693(86) 91601-1

508. Bernardi G, Carugno G, Chauveau J, Dicarlo F, Dris M, Dumarchez J, et al. FURTHER LIMITS ON HEAVY NEUTRINO COUPLINGS. Phys Lett. (1988) B203:332-34. doi: 10.1016/0370-2693(88)90563-1

509. Vilain P, Wilquet G, Petrak S, Beyer R, Flegel W, Grote H, et al. Search for heavy isosinglet neutrinos. Phys Lett. (1995) B343:453-58. doi: 10.1016/0370-2693(94)01422-9

510. Vaitaitis A, Drucker RB, Formaggio J, Adams T, Alton A, Avvakumov $\mathrm{S}$, et al. Search for neutral heavy leptons in a high-energy neutrino beam. Phys Rev Lett. (1999) 83:4943-6. doi: 10.1103/PhysRevLett. 83.4943

511. Adriani O, Aguilar-Benitez M, Ahlen S, Alcaraz J, Aloisio A, Alverson G, et al. Search for isosinglet neutral heavy leptons in Z0 decays. Phys Lett. (1992) B295:371-82.

512. Abreu P, Adam W, Adye T, Azhinenko I, Alekseev GD, Alemany R, et al. Search for neutral heavy leptons produced in Z decays. Z Phys. (1997) C74:57-71. doi: 10.1007/s002880050370

513. Sirunyan AM, Tumasyan A, Adam W, Ambrogi F, Asilar E, Bergauer T, et al. Search for a $W^{\prime}$ boson decaying to a $\tau$ lepton and a neutrino in proton-proton collisions at $\sqrt{s}=13 \mathrm{TeV}$. Phys Lett. (2018) B792:107. doi: 10.1016/j.physletb.2019.01.069

514. Aaboud M, Aad G, Abbott B, Abdinov O, Abeloos B, Abidi SH, et al. Search for new high-mass phenomena in the dilepton final state using $36 \mathrm{fb}^{-1}$ of proton-proton collision data at $\sqrt{s}=13 \mathrm{TeV}$ with the ATLAS detector. J High Energy Phys. (2017) 10:182. doi: 10.1007/JHEP10 (2017) 182

515. Collaboration $\mathrm{C}$. Search for a high-mass resonance decaying into a dilepton final state in $13 \mathrm{fb}^{-1}$ of pp collisions at $\sqrt{s}=13 \mathrm{TeV}$. (2016). Available online at: http://cds.cern.ch/record/2205764

516. Aaboud M, Aad G, Abbott B, Abdinov O, Abeloos B, Abidi SH, et al. Search for doubly charged Higgs boson production in multi-lepton final states with the ATLAS detector using proton-proton collisions at $\sqrt{s}=13 \mathrm{TeV}$. Eur Phys J. (2018) C78:199. doi: 10.1140/EPJC/S10052-018-5661-Z

517. Collaboration C. A search for doubly-charged Higgs boson production in three and four lepton final states at $\sqrt{s}=13 \mathrm{TeV}$. (2017).

518. Aaboud M, Aad G, Abbott B, Abdinov O, Abeloos B, Abhayasinghe DK, et al. Search for doubly charged scalar bosons decaying into same-sign $W$ boson pairs with the ATLAS detector. Submitted to: Eur Phys J. (2018) 79:58. doi: 10.1140/epjc/s10052-018-6500-y

519. Aaboud M, Aad G, Abbott B, Abbott DC, Abdinov O, Abeloos B, et al. Search for heavy long-lived multi-charged particles in proton-proton collisions at $\sqrt{s}=13 \mathrm{TeV}$ using the ATLAS detector. (2018) Phys Rev D. 99:052003. doi: 10.1103/PhysRevD.99.052003
520. Abbott LF, Wise MB. The effective hamiltonian for nucleon decay. Phys Rev. (1980) D22:2208. doi: 10.1103/PhysRevD.22.2208

521. Marciano WJ, Senjanovic G. Predictions of supersymmetric grand unified theories. Phys Rev. (1982) D25:3092. doi: 10.1103/PhysRevD. 25.3092

522. Dimopoulos S, Raby S, Wilczek F. Proton decay in supersymmetric models. Phys Lett. (1982) 112B:133. doi: 10.1016/0370-2693(82)90313-6

523. Nath P, Chamseddine AH, Arnowitt RL. Nucleon Decay in Supergravity Unified Theories. Phys Rev. (1985) D32:2348-58. doi: 10.1103/PhysRevD.32.2348

524. Arnowitt RL, Chamseddine AH, Nath P. NUCLEON DECAY BRANCHING RATIOS IN SUPERGRAVITY SU(5) GUTS. Phys Lett. (1985) 156B:215-9. doi: 10.1016/0370-2693(85)91512-6

525. Hisano J, Murayama H, Yanagida T. Nucleon decay in the minimal supersymmetric SU(5) grand unification. Nucl Phys. (1993) B402:46-84. doi: 10.1016/0550-3213(93)90636-4

526. Lucas V, Raby S. Nucleon decay in a realistic SO(10) SUSY GUT. Phys Rev. (1997) D55:6986-7009. doi: 10.1103/PhysRevD.55.6986

527. Goto T, Nihei T. Effect of RRRR dimension five operator on the proton decay in the minimal SU(5) SUGRA GUT model. Phys Rev. (1999) D59:115009. doi: 10.1103/PhysRevD.59.115009

528. Murayama H, Pierce A. Not even decoupling can save minimal supersymmetric SU(5). Phys Rev. (2002) D65:055009. doi: 10.1103/PhysRevD.65.055009

529. Dermisek R, Mafi A, Raby S. SUSY GUTs under siege: proton decay. Phys Rev. (2001) D63:035001. doi: 10.1103/PhysRevD.63.035001

530. Bajc B, Fileviez Perez P, Senjanovic G. Proton decay in minimal supersymmetric SU(5). Phys Rev. (2002) D66:075005. doi: 10.1103/PhysRevD.66.075005

531. Bajc B, Fileviez Perez P, Senjanovic G. Minimal supersymmetric SU(5) theory and proton decay: where do we stand? In: Beyond the Desert: Accelerator, Non-accelerator and Space Approaches Into the Next Millennium. Proceedings, $3 r d$ International Conference on particle physics beyond the standard model, Oulu, Finland, June 2-7, 2002), Oulu (2002). p. 131-9.

532. Emmanuel-Costa D, Wiesenfeldt S. Proton decay in a consistent supersymmetric SU(5) GUT model. Nucl Phys. (2003) B661:62-82. doi: 10.1016/S0550-3213(03)00301-8

533. Dorsner I, Fileviez Perez P. How long could we live? Phys Lett. (2005) B625:88-95. doi: 10.1016/j.physletb.2005.08.039

534. Fileviez Perez P. Fermion mixings versus $\mathrm{d}=6$ proton decay. Phys Lett (2004) B595:476-83. doi: 10.1016/j.physletb.2004.06.061

535. Nath P, Fileviez Perez P. Proton stability in grand unified theories, in strings and in branes. Phys Rept. (2007) 441:191-317. doi: 10.1016/j.physrep.2007.02.010

536. Abe K, Hayato Y, Iyogi K, Kameda J, Miura M, Moriyama S, et al. Search for proton decay via $p \rightarrow v v K^{+}$using 260 kiloton year data of Super-Kamiokande. Phys Rev. (2014) D90:072005. doi: 10.1103/PhysRevD.90.072005

537. Sussman S, Abe K, Bronner C, Hayato Y, Ikeda M, Iyogi K, et al. Dinucleon and nucleon decay to two-body final states with no hadrons in superkamiokande. arXiv:1811.12430 (2018).

538. Abe K, Abe K, Aihara H, Aimi A, Akutsu R, Andreopoulos C, et al. Hyper-kamiokande design report. arXiv:1805.04163 (2018).

539. Acciarri R, Acero MA, Adamowski M, Adams C, Adamson P, Adhikari S, et al. Long-baseline neutrino facility (LBNF) and deep underground neutrino experiment (DUNE). arXiv:1512.06148 (2015).

540. Kolešová H, Malinský M. Flavour structure of GUTs and uncertainties in proton lifetime estimates. (2016) Phys Rev. D99:035005. doi: 10.1103/PhysRevD.99.035005

541. Leurer M. A Comprehensive study of leptoquark bounds. Phys Rev. (1994) D49:333-42. doi: 10.1103/PhysRevD.49.333

542. Di Luzio L, Kirk M, Lenz A. Updated $B_{s}$-mixing constraints on new physics models for $b \rightarrow s \ell^{+} \ell^{-}$anomalies. Phys Rev. (2018) D97:095035. doi: 10.1103/PhysRevD.97.095035

543. Lees JP, Poireau V, Tisserand V, Wu SL, Grauges E, Palano A, et al. Measurement of an excess of $\bar{B} \rightarrow D^{(*)} \tau^{-} \bar{\nu}_{\tau}$ decays and implications for charged higgs bosons. Phys Rev. (2013) D88:072012. doi: 10.1103/PhysRevD.88.072012 
544. Hirose S, Iijima T, Adachi I, Adamczyk K, Aihara H, Said SA, et al. Measurement of the $\tau$ lepton polarization and $R\left(D^{*}\right)$ in the decay $\bar{B} \rightarrow D^{*} \tau^{-} \bar{\nu}_{\tau}$. Phys Rev Lett. (2017) 118:211801. doi: 10.1103/PhysRevLett.118.211801

545. Bailey JA, Bazavov A, Bernard C, Bouchard CM, DeTar C, Du D, et al. $\mathrm{B} \rightarrow \mathrm{D} \ell \mathrm{v}$ form factors at nonzero recoil and $\left|\mathrm{V}_{c b}\right|$ from 2+1-flavor lattice QCD. Phys Rev. (2015) D92:034506. doi: 10.1103/PhysRevD.92. 034506

546. Fajfer S, Kamenik JF, Nisandzic I. On the $B \rightarrow D^{*} \tau \bar{\nu}_{\tau}$ Sensitivity to New Physics. Phys Rev. (2012) D85:094025. doi: 10.1103/PhysRevD.85.094025

547. Bernlochner FU, Ligeti Z, Papucci M, Robinson DJ. Combined analysis of semileptonic $B$ decays to $D$ and $D^{*}: R\left(D^{(*)}\right),\left|V_{c b}\right|$, and new physics. Phys Rev. (2017) D95:115008. doi: 10.1103/PhysRevD.95.115008

548. Bigi D, Gambino P, Schacht S. A fresh look at the determination of $\left|V_{c b}\right|$ from $B \rightarrow D^{*} \ell v$. Phys Lett. (2017) B769:441-5. doi: 10.1016/j.physletb.2017.04.022

549. Amhis Y, Banerjee S, Ben-Haim E, Bernlochner F, Bozek A, Bozzi C, et al. Averages of $b$-hadron, $c$-hadron, and $\tau$-lepton properties as of summer 2016. Eur Phys J. (2016) C77:895. doi: 10.1140/epjc/s10052-017-5058-4

550. Aaij R, Adeva B, Adinolfi M, Ajaltouni Z, Akar S, Albrecht J, et al. Test of lepton universality with $B^{0} \rightarrow K^{* 0} \ell^{+} \ell^{-}$decays. J High Energy Phys. (2017) 08:055. doi: 10.1007/JHEP08(2017)055

551. Hiller G, Kruger F. More model-independent analysis of $b \rightarrow s$ processes. Phys Rev. (2004) D69:074020. doi: 10.1103/PhysRevD.69.074020

552. Bordone M, Isidori G, Pattori A. On the Standard Model predictions for $R_{K}$ and $R_{K^{*}}$. Eur Phys J. (2016) C76:440. doi: 10.1140/epjc/s10052-016-4274-7

553. D’Amico G, Nardecchia M, Panci P, Sannino F, Strumia A, Torre R, et al. Flavour anomalies after the $R_{K} *$ measurement. J High Energy Phys. (2017) 09:010. doi: 10.1007/JHEP09(2017)010

554. Capdevila B, Crivellin A, Descotes-Genon S, Matias J, Virto J. Patterns of New Physics in $b \rightarrow s \ell^{+} \ell^{-}$transitions in the light of recent data. J High Energy Phys. (2018) 01:093. doi: 10.1007/JHEP01(2018)093

555. Bhattacharya B, Datta A, London D, Shivashankara S. Simultaneous Explanation of the $R_{K}$ and $R\left(D^{(*)}\right)$ Puzzles. Phys Lett. 2015) B742:370-4. doi: 10.1016/j.physletb.2015.02.011

556. Freytsis M, Ligeti Z, Ruderman JT. Flavor models for $\bar{B} \rightarrow D^{(*)} \tau \bar{\nu}$. Phys Rev. (2015) D92:054018. doi: 10.1103/PhysRevD.92.054018

557. Buttazzo D, Greljo A, Isidori G, Marzocca D. B-physics anomalies: a guide to combined explanations. J High Energy Phys. (2017) 11:044. doi: 10.1007/JHEP11(2017)044

558. Angelescu A, Becirevic D, Faroughy DA, Sumensari O. Closing the window on single leptoquark solutions to the B-physics anomalies. J High Energy Phys. (2018) 10:183. doi: 10.1007/JHEP10(2018)183

559. Bauer M, Neubert M. Minimal Leptoquark Explanation for the $\mathrm{R}_{D^{(*)}}$ , $\mathrm{R}_{K}$, and $(g-2)_{g}$ Anomalies. Phys Rev Lett. (2016) 116:141802. doi: 10.1103/PhysRevLett.116.141802

560. Becirevic D, Sumensari O. A leptoquark model to accommodate $R_{K}^{\exp }<R_{K}^{\mathrm{SM}}$ and $R_{K^{*}}^{\exp }<R_{K^{*}}^{\mathrm{SM}}$. J High Energy Phys. (2017) 08:104. doi: 10.1007/JHEP08(2017)104

561. Becirevic D, Fajfer S, Košnik N, Sumensari O. Leptoquark model to explain the $B$-physics anomalies, $R_{K}$ and $R_{D}$. Phys Rev. (2016) D94:115021. doi: 10.1103/PhysRevD.94.115021

562. Becirevic D, Košnik N, Sumensari O, Zukanovich Funchal R. Palatable Leptoquark Scenarios for Lepton Flavor Violation in Exclusive $b \rightarrow$ $s \ell_{1} \ell_{2}$ modes. J High Energy Phys. (2016) 11:035. doi: 10.1007/JHEP11 (2016) 035

563. Chen $\mathrm{CH}$, Nomura $\mathrm{T}$, Okada $\mathrm{H}$. Excesses of muon $g-2, R_{D^{(*)}}$, and $R_{K}$ in a leptoquark model. Phys Lett. (2017) B774:456-64. doi: 10.1016/j.physletb.2017.10.005

564. de Medeiros Varzielas I, King SF. $R_{K^{(*)}}$ with leptoquarks and the origin of Yukawa couplings. J High Energy Phys. (2018) 11:100. doi: 10.1007/JHEP11(2018)100

565. Camargo-Molina JE, Celis A, Faroughy DA. Anomalies in Bottom from new physics in Top. Phys Lett. (2018) B784:284-93. doi: 10.1016/j.physletb.2018.07.051

566. Crivellin A, Müller D, Ota T. Simultaneous explanation of $\mathrm{R}\left(\mathrm{D}^{(*)}\right)$ and $\mathrm{b} \rightarrow s \mu^{+} \mu^{-}$: the last scalar leptoquarks standing. J High Energy Phys. (2017) 09:040. doi: 10.1007/JHEP09(2017)040
567. Cox P, Kusenko A, Sumensari O, Yanagida TT. SU(5) Unification with TeV-scale Leptoquarks. J High Energy Phys. (2017) 03:035. doi: 10.1007/JHEP03(2017)035

568. Doršner I, Fajfer S, Faroughy DA, Košnik N. The role of the $S_{3}$ GUT leptoquark in flavor universality and collider searches. J High Energy Phys. (2017) 10:188. doi: 10.1007/JHEP10(2017)188

569. Becirevic D, Doršner I, Fajfer S, Košnik N, Faroughy DA, Sumensari O. Scalar leptoquarks from grand unified theories to accommodate the $B$ physics anomalies. Phys Rev. (2018) D98:055003. doi: 10.1103/PhysRevD.98. 055003

570. Das D, Hati C, Kumar G, Mahajan N. Towards a unified explanation of $R_{D^{(*)}}, R_{K}$ and $(g-2)_{\mu}$ anomalies in a left-right model with leptoquarks. Phys Rev. (2016) D94:055034. doi: 10.1103/PhysRevD.94. 055034

571. Heeck J, Teresi D. Pati-Salam explanations of the B-meson anomalies. (2018). doi: 10.1007/JHEP12(2018)103

572. Aydemir U, Minic D, Sun C, Takeuchi T. B-decay anomalies and scalar leptoquarks in unified Pati-Salam models from noncommutative geometry. J High Energy Phys. (2018) 09:117. doi: 10.1007/JHEP09(2018)117

573. Aydemir U, Mandal T, Mitra S. A single TeV-scale scalar leptoquark in SO(10) grand unification and B-decay anomalies. (2019).

574. Faber T, Hudec M, Malinský M, Meinzinger P, Porod W, Staub F. A unified leptoquark model confronted with lepton non-universality in $B$ meson decays. Phys Lett. (2018) B787:159-66. doi: 10.1016/j.physletb.2018. 10.051

575. Sahoo S, Mohanta R, Giri AK. Explaining the $R_{K}$ and $R_{D^{(*)}}$ anomalies with vector leptoquarks. Phys Rev. (2017) D95:035027. doi: 10.1103/PhysRevD.95.035027

576. Crivellin A, Greub C, Saturnino F, Müller D. Importance of loop effects in explaining the accumulated evidence for New Physics in B Decays with a Vector Leptoquark. Phys Rev Lett. (2018) 122:011805. doi: 10.1103/PhysRevLett.122.011805

577. Sahoo S, Mohanta R. Impact of vector leptoquark on $\bar{B} \rightarrow \bar{K}^{*} l^{+} l^{-}$ anomalies. J Phys. (2018) G45:085003. doi: 10.1088/1361-6471/aaca12

578. Assad N, Fornal B, Grinstein B. Baryon number and lepton universality violation in leptoquark and diquark models. Phys Lett. (2018) B777:324-31. doi: 10.1016/j.physletb.2017.12.042

579. Calibbi L, Crivellin A, Li T. Model of vector leptoquarks in view of the B-physics anomalies. Phys Rev. (2018) D98:115002. doi: 10.1103/PhysRevD.98.115002

580. Di Luzio L, Greljo A, Nardecchia M. Gauge leptoquark as the origin of B-physics anomalies. Phys Rev. (2017) D96:115011. doi: 10.1103/PhysRevD.96.115011

581. Di Luzio L, Fuentes-Martin J, Greljo A, Nardecchia M, Renner S. Maximal Flavour Violation: a Cabibbo mechanism for leptoquarks. J High Energy Phys. (2018) 11:081. doi: 10.1007/JHEP11(2018)081

582. Greljo A, Stefanek BA. Third family quark-lepton unification at the TeV scale. Phys Lett. (2018) B782:131-8. doi: 10.1016/j.physletb.2018. 05.033

583. Bordone M, Cornella C, Fuentes-Martín J, Isidori G. Low-energy signatures of the PS ${ }^{3}$ model: from B-physics anomalies to LFV. J High Energy Phys. (2018) 10:148. doi: 10.1007/JHEP10(2018)148

584. Bordone M, Cornella C, Fuentes-Martin J, Isidori G. A three-site gauge model for flavor hierarchies and flavor anomalies. Phys Lett. (2018) B779:317-23. doi: 10.1016/j.physletb.2018.02.011

585. Faroughy DA, Greljo A, Kamenik JF. Confronting lepton flavor universality violation in B decays with high- $p_{T}$ tau lepton searches at LHC. Phys Lett. (2017) B764:126-34. doi: 10.1016/j.physletb.2016.11.011

586. Greljo A, Marzocca D. High- $p_{T}$ dilepton tails and flavor physics. Eur Phys $J$ (2017) C77:548. doi: 10.1140/epjc/s10052-017-5119-8

587. Schmaltz M, Zhong YM. The leptoquark Hunter's guide: large coupling. J High Energy Phys. (2018). doi: 10.1007/JHEP01(2019)132

588. Mandal T, Mitra S, Raz S. $R_{D^{(*)}}$ in minimal leptoquark scenarios: impact of interference on the exclusion limits from LHC data. Phys Rev D. (2018) 99:055028. doi: 10.1103/PhysRevD.99.055028

589. Greljo A, Martin Camalich J, Ruiz-Álvarez JD. The mono-tau menace: from $B$ decays to high- $p_{T}$ Tails. Phys Rev Lett. (2018) 122:131803. doi: 10.1103/PhysRevLett.122.131803 
590. Bansal S, Capdevilla RM, Delgado A, Kolda C, Martin A, Raj N. Hunting leptoquarks in monolepton searches. Phys Rev D. (2018) 98:015037. doi: 10.1103/PhysRevD.98.015037

591. Vignaroli N. Seeking LQs in the $\mathbf{t} \overline{\mathbf{t}}$ plus missing energy channel at the high-luminosity LHC. Phys Rev D. (2018) 99:035021. doi: 10.1103/PhysRevD.99.035021

592. Glashow SL, Iliopoulos J, Maiani L. Weak interactions with lepton-hadron symmetry. Phys Rev. (1970) D2:1285-92. doi: 10.1103/PhysRevD.2.1285

593. Petcov ST. The Processes mu $\rightarrow>$ e Gamma, mu $\rightarrow$ e e anti-e, Neutrino' $->$ Neutrino gamma in the Weinberg-Salam Model with Neutrino Mixing. Sov J Nucl Phys. (1977) 25:340.

594. Cheng TP, Li LF. Nonconservation of separate mu - lepton and e - lepton numbers in gauge theories with v+a currents. Phys Rev Lett. (1977) 38:381. doi: 10.1103/PhysRevLett.38.381

595. Bilenky SM, Petcov ST, Pontecorvo B. Lepton Mixing, mu $\rightarrow$ e + gamma Decay and Neutrino Oscillations. Phys Lett. (1977) 67B:309. doi: 10.1016/0370-2693(77)90379-3

596. Kuno Y, Okada Y. Muon decay and physics beyond the standard model. Rev Mod Phys. (2001) 73:151-202. doi: 10.1103/RevModPhys.73.151

597. Abada A, Krauss ME, Porod W, Staub F, Vicente A, Weiland C. Lepton flavor violation in low-scale seesaw models: SUSY and non-SUSY contributions. J High Energy Phys. (2014) 11:048. doi: 10.1007/JHEP11(20 14) 048

598. Bernabeu J, Santamaria A, Vidal J, Mendez A, Valle JWF. Lepton Flavor Nonconservation at High-Energies in a Superstring Inspired Standard Model. Phys Lett. (1987) B187:303-8. doi: 10.1016/0370-2693(87)91 100-2

599. Ilakovac A, Pilaftsis A. Flavor violating charged lepton decays in seesawtype models. Nucl Phys. (1995) B437:491. doi: 10.1016/0550-3213(94) 00567-X

600. Deppisch F, Kosmas TS, Valle JWF. Enhanced mu- - e- conversion in nuclei in the inverse seesaw model. Nucl Phys. (2006) B752:80-92. doi: 10.1016/j.nuclphysb.2006.06.032

601. Alonso R, Dhen M, Gavela MB, Hambye T. Muon conversion to electron in nuclei in type-I seesaw models. J High Energy Phys. (2013) 01:118. doi: 10.1007/JHEP01(2013)118

602. Barry J, Rodejohann W. Lepton number and flavour violation in TeV-scale left-right symmetric theories with large left-right mixing. J High Energy Phys. (2013) 09:153. doi: 10.1007/JHEP09(2013)153

603. Vicente A. Lepton flavor violation beyond the MSSM. Adv High Energy Phys. (2015) 2015:686572. doi: 10.1155/2015/686572

604. Grinstein B, Cirigliano V, Isidori G, Wise MB. Grand Unification and the Principle of Minimal Flavor Violation. Nucl Phys. (2007) B763:35-48. doi: 10.1016/j.nuclphysb.2006.11.005

605. Ciuchini M, Masiero A, Paradisi P, Silvestrini L, Vempati SK, Vives O. Soft SUSY breaking grand unification: Leptons versus quarks on the flavor playground. Nucl Phys. (2007) B783:112-42. doi: 10.1016/j.nuclphysb.2007.05.032

606. Hisano J, Moroi T, Tobe K, Yamaguchi M. Lepton flavor violation via right-handed neutrino Yukawa couplings in supersymmetric standard model. Phys Rev. (1996) D53:2442-59. doi: 10.1103/PhysRevD. 53.2442

607. Arganda E, Herrero MJ. Testing supersymmetry with lepton flavor violating tau and mu decays. Phys Rev. (2006) D73:055003. doi: 10.1103/PhysRevD.73.055003

608. Hirsch M, Joaquim FR, Vicente A. Constrained SUSY seesaws with a $125 \mathrm{GeV}$ Higgs. J High Energy Phys. (2012) 11:105. doi: 10.1007/JHEP11(2012)105

609. Rossi A. Supersymmetric seesaw without singlet neutrinos: Neutrino masses and lepton flavor violation. Phys Rev. (2002) D66:075003. doi: 10.1103/PhysRevD.66.075003

610. Deppisch F, Valle JWF. Enhanced lepton flavor violation in the supersymmetric inverse seesaw model. Phys Rev. (2005) D72:036001. doi: 10.1103/PhysRevD.72.036001

611. Hirsch M, Valle JWF, Porod W, Romao JC, Villanova del Moral A. Probing minimal supergravity in type I seesaw with lepton flavour violation at the LHC. Phys Rev. (2008) D78:013006. doi: 10.1103/PhysRevD.78. 013006
612. Esteves JN, Romao JC, Hirsch M, Staub F, Porod W. Supersymmetric typeIII seesaw: lepton flavour violating decays and dark matter. Phys Rev. (2011) D83:013003. doi: 10.1103/PhysRevD.83.013003

613. Dreiner HK, Kramer M, O'Leary B. Bounds on R-parity violating supersymmetric couplings from leptonic and semi-leptonic meson decays. Phys Rev. (2007) D75:114016. doi: 10.1103/PhysRevD.75.114016

614. de Gouvea A, Lola S, Tobe K. Lepton flavor violation in supersymmetric models with trilinear R-parity violation. Phys Rev. (2001) D63:035004. doi: 10.1103/PhysRevD.63.035004

615. Arhrib A, Cheng Y, Kong OCW. Comprehensive analysis on lepton flavor violating Higgs boson to $\mu^{\mp} \tau^{ \pm}$decay in supersymmetry without $R$ parity. Phys Rev. (2013) D87:015025. doi: 10.1103/PhysRevD.87.015025

616. Romao JC, Rius N, Valle JWF. Supersymmetric signals in muon and $\tau$ decays. Nucl Phys. 1991) B363:369-84. doi: 10.1016/0550-3213(91)80025-H

617. Hirsch M, Vicente A, Meyer J, Porod W. Majoron emission in muon and tau decays revisited. Phys Rev. (2009) D79:055023. doi: 10.1103/PhysRevD.79.079901

618. Raidal M, van der Schaaf A, Bigi I, Mangano ML, Semertzidis Y, Abel S, et al. Flavour physics of leptons and dipole moments. Eur Phys J. (2008) C57:13-182. doi: 10.1140/epjc/s10052-008-0715-2

619. Abada A, Toma T. Electric dipole moments of charged Leptons with sterile fermions. J High Energy Phys. (2016) 02:174. doi: 10.1007/JHEP02(2016)174

620. Dimopoulos S, Hall LJ. Electric dipole moments as a test of supersymmetric unification. Phys Lett. (1995) B344:185-92. doi: 10.1016/0370-2693(94)01572-T

621. Romanino A, Strumia A. Electron and muon electric dipoles in supersymmetric scenarios. Nucl Phys. (2002) B622:73-94. doi: 10.1016/S0550-3213(01)00607-1

622. Jung M, Pich A. Electric dipole moments in two-higgs-doublet models. J High Energy Phys. (2014) 04:076. doi: 10.1007/JHEP04(2014)076

623. Stockinger D. The muon magnetic moment and supersymmetry. $J$ Phys (2007) G34:R45-92. doi: 10.1088/0954-3899/34/2/R01

624. Dekens W, De Vries J, Jung M, Vos KK. The phenomenology of electric dipole moments in models of scalar leptoquarks. J High Energy Phys. (2018) 01:069. doi: 10.1007/JHEP01(2019)069

625. Fuyuto K, Ramsey-Musolf M, Shen T. Electric dipole moments from CP-Violating scalar leptoquark interactions. Phys Lett. (2019) B788:52-7. doi: 10.1016/j.physletb.2018.11.016

626. Baldini AM, Bao Y, Baracchini E, Bemporad C, Berg F, Biasotti M, et al. Search for the lepton flavour violating decay $\mu^{+} \rightarrow \mathrm{e}^{+} \gamma$ with the full dataset of the MEG experiment. Eur Phys J. (2016) C76:434. doi: 10.1140/epjc/s10052-016-4271-x

627. Aubert B, Karyotakis Y, Lees JP, Poireau V, Prencipe E, Prudent X, et al. Searches for Lepton Flavor Violation in the Decays $\tau^{+-} \rightarrow$ $e^{+-}$gamma and $\tau^{+-} \rightarrow \mu^{+-}$gamma. Phys Rev Lett. (2010) 104:021802. doi: 10.1103/PhysRevLett.104.021802

628. Hayasaka K, Abe K, Adachi I, Anipko HAD, Arinstein K, et al. New Search for tau $->$ mu gamma and tau $->$ e gamma Decays at Belle. Phys Lett. (2008) B666:16-22. doi: 10.1016/j.physletb.2008.06.056

629. Bellgardt U, Otter G, Eichler R, Felawka L, Niebuhr C, Walter HK, et al. Search for the Decay mu+ - - > e+ e+ e-. Nucl Phys. (1988) B299:1-6. doi: 10.1016/0550-3213(88)90462-2

630. Lees JP, Poireau V, Prencipe E, Tisserand V, Tico JG, Grauges E, et al. Limits on tau Lepton-Flavor Violating Decays in three charged leptons. Phys Rev. (2010) D81:111101. doi: 10.1103/PhysRevD.81.111101

631. Hayasaka K, Inami K, Miyazaki Y, Arinstein K, Aulchenko V, Aushev T, et al. Search for Lepton Flavor Violating Tau Decays into Three Leptons with 719 Million Produced Tau+Tau- Pairs. Phys Lett. (2010) B687:139-43. doi: 10.1016/j.physletb.2010.03.037

632. Aad G, Abbott B, Abdallah J, Abdinov O, Aben R, Abolins M, et al. Probing lepton flavour violation via neutrinoless $\tau \longrightarrow 3 \mu$ decays with the ATLAS detector. Eur Phys J. (2016) C76:232. doi: 10.1140/epjc/s10052-016-4041-9

633. Aaij R, Beteta CA, Adeva B, Adinolfi M, Affolder A, Ajaltouni Z, et al. Search for the lepton flavour violating decay $\tau^{-} \rightarrow \mu^{-} \mu^{+} \mu^{-}$.J High Energy Phys. (2015) 02:121. doi: 10.1007/JHEP02(2015)121

634. Kaulard J, Dohmen C, Haan H, Honecker W, Junker D, Otter G, et al. Improved limit on the branching ratio of $\mu->\mathrm{e}+$ conversion on titanium. Phys Lett. (1998) B422:334-8. doi: 10.1016/S0370-2693(97)01423-8 
635. Honecker W, Dohmen C, Haan H, Junker D, Otter G, Starlinger M, et al. Improved limit on the branching ratio of $\mu \rightarrow \mathrm{e}$ conversion on lead. Phys Rev Lett. (1996) 76:200-3. doi: 10.1103/PhysRevLett. 76.200

636. Bertl WH, Rosenbaum F, Ryskulov NM, Engfer R, Hermes EA, Kurz G, et al. A Search for muon to electron conversion in muonic gold. Eur Phys J. (2006) C47:337-46. doi: 10.1140/epjc/s2006-02582-x

637. Andreev V, Ang DG, DeMille D, Doyle JM, Gabrielse G, Haefner J, et al. Improved limit on the electric dipole moment of the electron. Nature. (2018) 562:355-60. doi: 10.1038/s41586-018-0599-8

638. Bennett GW, Bousquet B, Brown HN, Bunce G, Carey RM, Cushman P, et al. An Improved Limit on the Muon Electric Dipole Moment. Phys Rev. (2009) D80:052008. doi: 10.1103/PhysRevD.80.052008

639. Inami K, Abe K, Abe R, Abe T, Adachi I, Aihara H, et al. Search for the electric dipole moment of the tau lepton. Phys Lett. (2003) B551:16-26. doi: 10.1016/S0370-2693(02)02984-2

640. Hanneke D, Fogwell S, Gabrielse G. New measurement of the electron magnetic moment and the fine structure constant. Phys Rev Lett. 2008) 100:120801. doi: 10.1103/PhysRevLett.100.120801

641. Bennett GW, Bousquet B, Brown HN, Bunce G, Carey RM, Cushman P, et al. Final report of the muon E821 anomalous magnetic moment measurement at BNL. Phys Rev. (2006) D73:072003. doi: 10.1103/PhysRevD.73. 072003

642. Blondel A, Bravar A, Pohl M, Bachmann S, Berger N, Kiehn M, et al. Research proposal for an experiment to search for the decay $\mu \rightarrow$ eee. arXiv:1301.6113 (2013).

643. Kurup A. The cOherent muon to electron transition (COMET) experiment. Nucl Phys Proc Suppl. (2011) 218:38-43. doi: 10.1016/j.nuclphysbps.2011.06.008

644. Kutschke RK. The Mu2e Experiment at Fermilab. In: Proceedings, 31st International Conference on Physics in collisions (PIC 2011). Vancouver, BC. (2011). Available online at: http://lss.fnal.gov/archive/2011/conf/fermilabconf-11-887-cd.pdf.

645. Chapelain A. The Muon g-2 experiment at Fermilab. EPJ Web Conf. (2017) 137:08001. doi: 10.1051/epjconf/201713708001

646. Furry WH. On transition probabilities in double beta-disintegration. Phys Rev. (1939) 56:1184-93. doi: 10.1103/PhysRev.56.1184

647. Agostini M, Allardt M, Bakalyarov AM, Balata M, Barabanov I, Baudis L, et al. Background-free search for neutrinoless double- $\beta$ decay of ${ }^{76} \mathrm{Ge}$ with GERDA. Nature. (2017) 544:47. doi: 10.1038/nature21717

648. Gando A, Gando Y, Hachiya T, Hayashi A, Hayashida S, Ikeda $\mathrm{H}$, et al. Search for majorana neutrinos near the inverted mass hierarchy region with kamLAND-zen. Phys Rev Lett. (2016) 117:082503. doi: 10.1103/PhysRevLett.117.082503

649. Schechter J, Valle JWF. Neutrinoless double beta decay in $\mathrm{SU}(2) \times \mathrm{U}(1)$ theories. Phys Rev. (1982) D25:2951. doi: 10.1103/PhysRevD.25.2951

650. Nieves JF. Dirac and pseudodirac neutrinos and neutrinoless double beta decay. Phys Lett. (1984) 147B:375-9. doi: 10.1016/0370-2693(84) 90136-9

651. Takasugi E. Can the neutrinoless double beta decay take place in the case of dirac neutrinos? Phys Lett. (1984) 149B:372-6. doi: 10.1016/0370-2693(84)90426-X
652. Pas H, Hirsch M, Klapdor-Kleingrothaus HV, Kovalenko SG. Towards a superformula for neutrinoless double beta decay. Phys Lett. (1999) B453:194-8. doi: 10.1016/S0370-2693(99)00330-5

653. Pas H, Hirsch M, Klapdor-Kleingrothaus HV, Kovalenko SG. A Superformula for neutrinoless double beta decay. 2. The Short range part. Phys Lett. (2001) B498:35-9. doi: 10.1016/S0370-2693(00)01359-9

654. Deppisch FF, Graf L, Harz J, Huang WC. Neutrinoless double beta decay and the baryon asymmetry of the universe. Phys Rev. (2018) D98:055029. doi: 10.1103/PhysRevD.98.055029

655. Cirigliano V, Dekens W, de Vries J, Graesser ML, Mereghetti E. A neutrinoless double beta decay master formula from effective field theory. J High Energy Phys. (2018) 12:097. doi: 10.1007/JHEP12 (2018)097

656. Graf L, Deppisch FF, Iachello F, Kotila J. Short-range neutrinoless double beta decay mechanisms. Phys Rev. (2018) D98:095023. doi: 10.1103/PhysRevD.98.095023

657. Deppisch FF, Hirsch M, Pas H. Neutrinoless double beta decay and physics beyond the standard model. J Phys. (2012) G39:124007. doi: 10.1088/0954-3899/39/12/124007

658. Hirsch M, Klapdor-Kleingrothaus HV, Kovalenko SG. New lowenergy leptoquark interactions. Phys Lett. (1996) B378:17-22. doi: 10.1016/0370-2693(96)00419-4

659. Hirsch M, Klapdor-Kleingrothaus HV, Kovalenko SG. New leptoquark mechanism of neutrinoless double beta decay. Phys Rev. (1996) D54:R420710. doi: 10.1103/PhysRevD.54.R4207

660. Mohapatra RN. New contributions to neutrinoless double beta decay in supersymmetric theories. Phys Rev. (1986) D34:3457-61. doi: 10.1103/PhysRevD.34.3457

661. Hirsch M, Klapdor-Kleingrothaus HV, Kovalenko SG. New constraints on Rparity broken supersymmetry from neutrinoless double beta decay. Phys Rev Lett. (1995) 75:17-20. doi: 10.1103/PhysRevLett.75.17

662. Hirsch M, Klapdor-Kleingrothaus HV, Kovalenko SG. Supersymmetry and neutrinoless double beta decay. Phys Rev. (1996) D53:1329-1348. doi: 10.1103/PhysRevD.53.1329

663. Hirsch M, Klapdor-Kleingrothaus HV, Kovalenko SG. On the SUSY accompanied neutrino exchange mechanism of neutrinoless double beta decay. Phys Lett. (1996) B372:181-6. doi: 10.1016/0370-2693(96)00050-0

664. Pas H, Hirsch M, Klapdor-Kleingrothaus HV. Improved bounds on SUSY accompanied neutrinoless double beta decay. Phys Lett. (1999) B459:450-4. doi: 10.1016/S0370-2693(99)00711-X

Conflict of Interest Statement: The authors declare that the research was conducted in the absence of any commercial or financial relationships that could be construed as a potential conflict of interest.

Copyright () 2019 Croon, Gonzalo, Graf, Košnik and White. This is an open-access article distributed under the terms of the Creative Commons Attribution License (CC $B Y)$. The use, distribution or reproduction in other forums is permitted, provided the original author(s) and the copyright owner(s) are credited and that the original publication in this journal is cited, in accordance with accepted academic practice. No use, distribution or reproduction is permitted which does not comply with these terms. 